\title{
Sensitive estimations of pituitary glycoprotein hormones : clinical and scientific applications
}

Citation for published version (APA):

Menheere, P. P. C. A. (1989). Sensitive estimations of pituitary glycoprotein hormones : clinical and scientific applications. [Doctoral Thesis, Maastricht University]. Rijksuniversiteit Limburg. https://doi.org/10.26481/dis.19890615pm

Document status and date:

Published: 01/01/1989

DOI:

10.26481/dis. $19890615 \mathrm{pm}$

Document Version:

Publisher's PDF, also known as Version of record

\section{Please check the document version of this publication:}

- A submitted manuscript is the version of the article upon submission and before peer-review. There can be important differences between the submitted version and the official published version of record.

People interested in the research are advised to contact the author for the final version of the publication, or visit the DOI to the publisher's website.

- The final author version and the galley proof are versions of the publication after peer review.

- The final published version features the final layout of the paper including the volume, issue and page numbers.

Link to publication

\footnotetext{
General rights rights.

- You may freely distribute the URL identifying the publication in the public portal. please follow below link for the End User Agreement:

www.umlib.nl/taverne-license

Take down policy

If you believe that this document breaches copyright please contact us at:

repository@maastrichtuniversity.nl

providing details and we will investigate your claim.
}

Copyright and moral rights for the publications made accessible in the public portal are retained by the authors and/or other copyright owners and it is a condition of accessing publications that users recognise and abide by the legal requirements associated with these

- Users may download and print one copy of any publication from the public portal for the purpose of private study or research.

- You may not further distribute the material or use it for any profit-making activity or commercial gain

If the publication is distributed under the terms of Article $25 \mathrm{fa}$ of the Dutch Copyright Act, indicated by the "Taverne" license above, 


\section{Sensitive Estimations of Pituitary Glycoprotein Hormones:}

Clinical and Scientific Applications. 
coverphoto:

Anno 1953 no sensitive assays for thyrotrophin were available. In contrast, thyroid hormone status was assessed using devices measuring Basal Metabolic Rate.

(photograph by courtesy of Jouan, laboratory equipment, Saint-Herblain, France).

grafische verzorging:

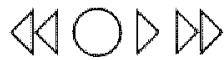

V.C.P.

Visual Communication Productions, Maastricht.

druk:

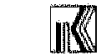




\section{Sensitive Estimations \\ of \\ Pituitary Glycoprotein Hormones:}

Clinical and Scientific Applications.

\section{Proefschrift}

ter verkrijging van de graad van doctor

aan de Rijksuniversiteit Limburg te Maastricht, op gezag van de Rector Magnificus, Prof.Dr.F.I.M.Bonke, volgens het besluit van het College van Dekanen, in het openbaar te verdedigen op donderdag 15 juni 1989 om 16.00 uur

door

Paulus Petrus Cornelis Antonius Menheere

geboren te Venray 
Promotores:

Prof.Dr.P.J. Brombacher

Prot.Dr.W. van der Slik (R.U. Groningen)

Co-promotor:

Dr.H.A.Keizer

Beoordelingscommissie:

Prot.Dr.P.S. Reneman,voorzitter

Prot.Dr.J.A. Flendrig

Prot.Dr.Dr.H. Greiling (RWTH, Aken, B.R.D.)

Prot.Dr.J. de Haan

Prof.Dr.J.D. Veldhuis (University of Virginia,

Charlottesville, U.S.A.)

De uitgave van dit proefschrift is mogelijk gemaakt door financiële steun van de Stichting Dr. Ir. J.H. J. van der Laar te Heerlen en de Stichting ter Bevordering van de Klinische Chemie in Nederland. 
aan:

milin ouders

aan:

Marie-Francoise

Cécile, Vincent, Paul en Yves 

Chapter 1: The pituitary as central controller.

1.1. Introduction. 13

1.2. Characteristics of negative feedback systems. 14

1.3. Prinary dysregulation of hormonal states. 14

1.4. Secondary and tertiary dysregulation of hormonal states. 16

1.5. Sensitive measurements of stimulating hormones. 18

Chapter 2: Sensitive Estimations of Glycoprotein Concentrations

2.1. General Principles of Immunoassay. 25

2.2. Ligand-Receptor Assays. 25

2.3. Antibodies and their impacts on specificity. 28

2.4. Tracers and their impacts on sensitivity. 32

2.5. Analytical Precision and Accuracy. 39

2.6. Sensitive estination of Thyroid Stimulating Hormone. 45

2.7. Sensitive estimation of Luteinizing Hormone. 54

Chapter 3: Clinical Applications of Sensitive TSH assays.

3.1. Effect of ritodrine on thyroid hormone concentrations. 59

3.2. Thyroid hormone status during the follicular and luteal 64

3.3. End Organ responses, clinical judgement or biochemical 68 control of thyroxine replacement therapy in hypothyroidism?

Chapter 4: Introduction to Pulsatility, Pulse Detection and changes in Pulsatility due to Exercise.

4.1. Endocrine Rhythms. 75

4.2. Exercise induced changes in endocrinology. 77

4.3. Pulse Detection. 78

4.4. Pulsatility of TSH.

4.5. Puisatility of $\mathrm{LH}$. 83 
Chapter 5: Pulsatility of pituitary glycoproteins.

5.1. Pulsatility of Luteinizing hormone. 89

5.2. Pulsatility of thyroid stimulating hormone. 109

Chapter 6: General Discussion. 129

$\begin{array}{lr}\text { Summary } & 133\end{array}$

$\begin{array}{ll}\text { Samenvatting } & 137\end{array}$

$\begin{array}{ll}\text { Dankwoord } & 141\end{array}$

Curriculum Vitae 143 


\section{General Introduction}

With the rapid development of molecular biology in the last decades, it has increasingly become evident that metabolic processes either at cellutar or at organ level are governed by regulatory mechanisms. This implicates both stimulation and inhibition of events at the molecular level, i.e. biochemical reactions. Many of these are controlled by circumstantial data at the (sub)cellular level (such as $\mathrm{pH}$ and the type of avallable buffer), the absence or presence and concentration of cofactors and ions, the production and consumption of substrates for generally enzymatically controlled reactions and, last but not least, the possibility of transport of compounds at the molecular level. By these (and other) influences, chemical equilibrium following the law of mass action is often achieved, leading to stability at higher levels (such as cellular) and in metabolic status and function of organs. It goes without saying that the possibility of obtaining information on physiological processes based on these biochemical events depends primarily on the degree wherein information can be obtained, i.e. on the achievements of highly advanced analysis of key-substances describing and regulating the reactions. Moreover, the analyses must be made without actually influencing or disturbing the processes to be studied. The basis of investigations in this field is always the availability and reliability of the necessary sensitivity, specificity, precision, accuracy and reproducibility of the analyses. The boundaries of obtainable information are set by these demands.

The present study deals with the growing insights in the regulatory mechanisms as performed and surveyed by two pituitary hormones for which ultra sensitive procedures of analytical determination have recently become available. Far from claiming an exhaustive complete description of all physiological processes in which these hormones play a more or less important role, we will describe the basic analytical requirements and the applicablity on some selected clinical and scientific implications.

Living beings of higher order benefit from two distinct control mechanisms permitting specialized tissues to function in integrated fashion. Firstly, there is the nervous system. Peripheral tissues transmit and receive signals to and from the brain via dedicated communication channels, creating an efficient and rapid information exchange necessary for the coordination of all possible functions of the tissues. Secondlly, there is the endocrine system, using chemical mediators (hormones) rather than electro-chemical signals. There is no specific provision for the transport of information, rendering the system completely dependent on the systemic blood circulation. However, due to the presence of blood in almost every body compartment, the influence of the endocrine system is even more omnipresent than that of the nervous system. Since periplieral tissues may answer to central regullation with the production of dedicated hormones, the endocrine system has, just like the nervous system, a two-way traffic of information available. However, the transport medium has the disadvantage of being relatively slow whereas time is necessary for the hormones to be synthesized and secreted in the blood stream. In consequence, the time course of the information exchange is appreciably longer than that of the nervous system. 
Both the nervous and the endocrine systems function essentially as feedback systems: the degree of regulation is controlled precisely by one or more mechanisms, back-regulated themselves by the regulated function.

It can be stated that virtually all body tissues are controlled by both systems, although the degree of control varies. Some glands seems to be dominantly controlled by the endocrine system (e.g. the thyroid and the gonads), whereas others are regulated almost exclusively by the nervous system (e.g. the adrenal medulla). Both systems are tightly coupled at the hypothalamic-pituitary level. Within this axis the various signals are integrated to result in one effective control unit.

Knowedge of the interference of the hypothalamic function with the pituitary function provides basic information necessary to the underslanding of a variety of (patho)physiological conditions. Actually much intormation has been gathered about the functioning of the pituitary: most of the stimulating (among which TSH, LH, FSH, ACTH) and inhibiting (among which dopamine, thyroid and sex steroid hormones and recenilly inhibin) mechanisms have been explored and this has resulted in better treatment of endocrine disorders.

However , knowledge of one part of an integral system amplifies the desire for information on the other part, i.e. the hypothalamus. The accessibility of this organ is much less than that of the pituitary. This is true for physical access (surgical, $X$-rays, isotope scanning) as well as chemical access since the hormones of hypothalamic origin are transported to the pituitary via a local portal system. Furthermore, these hormones have very short half-lives and seem to be very unstable in-vivo. Virtually, there seems to be no easy direct access to the hypothalamus. However, an indirect access is provided by detailed study of the exact behaviour of pituitary hormones. This is especially true, since rapid variations depend more on hypothalamic control rather than upon variations of peripheral hormone concentrations. The small variations in the concentration of pituitary hormones provide a window through which hypothalamic activity may be studied. The detection of such small variations lays much emphasis on the assay methodology. In fact, pituitary hormones (normally) are secreted in very small amounts. Large parts of the molecules have exact identical structures, while the remaining parts show great similarity. Accordingly, assays to quantify pituitary hormones must be very sensitive and very specific in order to be valid in the study of the controlling mechanisms. The introduction of monoclonal antibodies and the improvement in labelling techniques have contributed to the development of assays that can satisty the above mentioned criteria.

In this study, the results are presented of research that has been performed with the two first available newly developed assays, one for TSH and one for LH.

In chapter 1 , the central role of the pituitary as 'controller' in the endocrine system of the mammalian body has been reviewed, with special emphasis on the newer understanding that can be obtained through more detailed knowledge of the processes of production and feedback regulation of pituitary glycoproteins. Hitherto less understood phenomena can now be explained because of increased insight in the pituitary physiology such as the pulsatory production of hormones.

The basis of this new information can be found in the advancements of analytical biochemistry, more specifically in the field of immuno-chemical analysis.

The possibility of obtaining highly selective antibodies to glycoproteins and the subsequent developments using these antibodies as analytical reagents fulfilled the 
absolutely necessary requirements for sensitive and selective analysis, even increased by newly available techniques of detection "i.e. immunometric assays using antibody labelling techniques without loss of immunospecificity. A thorough description of these achievements and the inherent pitfalls is given in chapter 2 . With regard to TSH some possible applications of sensitive estimations have been investigated (chapter 3 ):

- the supposed development of hyperthyroidism during the suppression of premature labour in pregnant women;

- the relation between thyroid hormone status and phase of menstrual cycle with special interest in the explanation of the rise in body temperature after ovulation;

- the relation between thyroid hormone suppletion and functional parameters in order to provide a good method for the establishment of the most appropriate dose of replacement therapy.

In all these cases, estimations of thyroid hormone status by conventional methods including RIA or RIA-like methods for TSH, are insufficient, since they are lacking in sensitivity and sometimes even in specificity.

It will be shown that, besides the many advantages that have already been published, the new TSH assay permits the investigation of some physiological processes in much more detail.

With regard to $\mathrm{LH}$, the situation is much more complicated: firstly, low concentrations of LH enjoyed little clinical interest, undoubtedly caused by the simple fact that the lower detection limit of the conventional assays was above the lower limit of normal. No distinction could be made between LH-levels in hypo- and eugonadotropic states.

Secondly, the existence of at least five iso-hormones for LH has been described. The distribution of these iso-hormones between men, women, boys and girls varies. To date, we are unable to indicate the role of each of them in the overall biological activity of $\mathrm{LH}$. In our study, it has been verified that the new assay for LH produces results that correlate with the apparent status of the patient (chapter 2). Unfortunately, for the moment we were unable to demonstrate the clinical advantages of the increased sensitivity and specificity of the LH assays. However, the new LH assay has been used to study the regulatory mechanisms of $\mathrm{LH}$ concentration in more detail, since much knowledge is lacking about the significance of episodic hormone secretion under various conditions.

One aspect of pituitary hormonal regulation that has recently drawn much altention is the episodic or pulsatile nature of secretion of pituitary glycoproteins into the blood stream. The importance of such a secretory pattern is not yet fully understood, although pulsatile hormone administration has already found its application in clinical situations. The study of $\mathrm{LH}$ related phenomena has long suffered from the lack of sensitivity of the conventional biochemical assays and they had a lower detection limit even above the lowest possible physiological plasma concentrations. Another complication of the study of pulsatility was the need of substantially large plasma samples for analysis, which raised ethical problems because pulsatility studies sui generis require frequent sampling.

Use of the new techniques as described in this thesis overcomes some of these difficulties. For a better understanding of the effects of episodic hormone secretion and/or production, however, not only advanced analysis but also better understanding of the phenomenon of pulsatility itself is necessary.

In chapter 4 a treatment of this problem is given based on the latest assumptions now 
valid in this field. Programmes for the study of pulse detection are presented with special emphasis on the errors often encountered in this complicated type of imestigation.

One of the conditions which might influence episodic LH (and TSH) secretion is acute physical exercise and training. In fact, since physical exercise has many influences on the endocrine system (chapter 4) and the description of the pulsatile behaviour of a hormone in terms of pulse frequency, pulse amplitude and pulse duration in relation to physiological events may be informative about the hypothalamic regulation, the research has been concentrated on the effects of chronic and acute physical exercise. Chapter 5 contains the results of our studies of these effects on the pulsatile secretion paiterns of both $\mathrm{LH}$ and TSH. Too many details of the mechanism of hypothalamic action are still unknown. The proposed results of the pulsatile pattern of two pituitary hormones form an eariy step irn the unravelling of hypothalamic mechanisms. 


\section{Chapter 1}

\section{The pituitary as central controller.}

\subsection{Introduction}

It has long been recognized that many endocrine glands such as the thyroid and the gonads are regulated by the pituitary.

Thyroid hormone production is impossible without the presence of thyroid stimulating hormone (TSH), while the production of sex steroids by the gonads is primarly controlled by the luteinizing hormone $(\mathrm{LH})$, and follicle stimulating hormone (FSH). Together with the pituitary, both organs form two distinct, so called negative feedback mechanisms.

The secretion of the stimulating hormones by the pituitary is positively influenced by a lowering of the concentration of the hormone produced by the target organ, whereas in the latter the production and secretion of the hormone are stimulated by the stimulating hormone (figure 1.1).

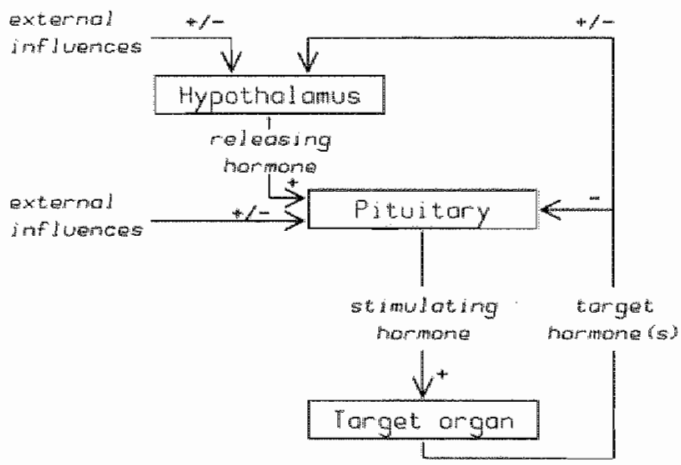

Figure 1.1: Two step negalive feedback mechanism.

The "set-point" of the pituitary is different for each individual and at least for TSH and LH can be influenced by the hypothalamus. In fact, the hypothalamus produces 'releasing hormones" (thyrotrophin releasing hormone, TRH and gonadotrophin releasing hormone, Gn- RH) which influence the pituitary secretion of TSH, LH and FSH. Depending on the plasma concentrations of the target organ hormones a positive feedback (at 'normal" hormone concentrations) or a negative feedback (target organ hormones outside the set-points) can be the case. Later on in this chapter the regulation of the hormone secretion by the various organs will be discussed in more detail.

The production of another pituitary hormone, adrenocorticotropic hormone is also submitted to a negative feedback mechanism with the adrenals. The regulatory mechanism of the secretion and release of two more pituitary hormones, growth hormone and prolactin, are governed by growth hormone releasing factor (GRH) and prolactir' inhibiting factor (Pr-IF). However, these hormones will not be discussed in this thesis. 


\subsection{Characteristics of negative feedback systems.}

A particular characteristic of an intact pituitary feedback system is the absence of the stimulating hormone in the peripheral circulation in cases of overproduction of hormones by the target organ (hyper hormonal state) (I). In contrast the concentrations of a stimulating hormone are elevated if the target organ is not able to satisfy the production need for peripheral activity (hypo hormonal state).

In the normal state, there will be an equilibrium between the positive and negatwe influences within the regulatory mechanism, resulting in a stable target cell produced hormone concentration.

Dystunction of the feedback mechanism will result in hyper hormonal or hypo hormonal states. The cause of hormonal dysgenesis can be situated at three levels within the hypothalamic-pituitary-target cell-axis :

- primary, the defect can be located within the target organ;

- secondary, the pituitary can be at the origin of the dysfunction, and

- tertiary, the cause of the disease can be located within the hypothalamus, or higher cerebrall centres.

\subsection{Primary dysregulation of hormonal states.}

\subsubsection{Primary hypo hormonal states}

Considering ihe thyroid and the gonads, primary hypo hormonal states are characterized by hormone concentrations below normal. Due to the feedback reguiatory mechanism, the pituitary will raise the concentrations of the stimulating hormones.

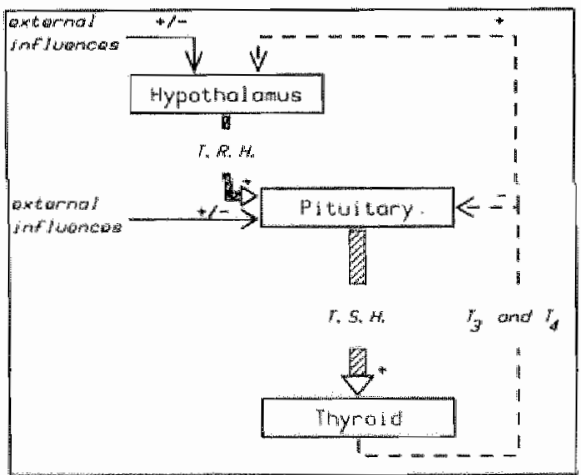

Figure 1.2: Disturbances of "leedback mechanism a: in primary hypothyroidism.

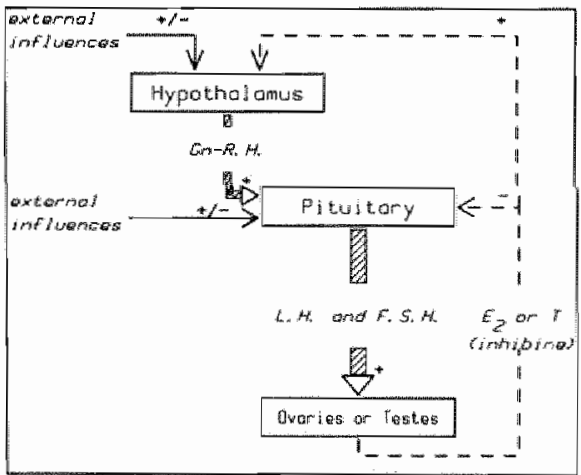

$\mathrm{b}$ : in hypergonadotropic hypogonadism.

Thus, in primary hypothyroidism, the thyroid hormones ( $T_{4}$ and sometimes $T_{3}$ ) will be lower than normal and TSH will be elevated (figure 1.2a).

In primary hypogonadism (normally called hypergonadotropic hypogonadism), the 
concentrations of LH and FSH will be high, while in women estradiol and in men lestosterone will be low (figure 1.2b).

Generally, there is a large overlap of the target homone concentrations as measured in normal individuals and the values obtained in patients with hypo hormonal states. Hence, it is difficult to classify an individual whin one of both groups if only the values of the target homone concentrations are awailable

The assay of TSH in patients with suspected hypothyroidism and of LH and/or FSH in patients with suspected hypogonadism permits in most cases a correct classification between the hypo hormonal and the normal hormonal state. The more pronounced the deficiency of the target hormone, the more the blood concentration of the corresponding stimulating hormone will be elevated. The overlap of the distribution of the concentrations of the stimulating hormones in both groups is much smaller than that of the target hormone concentrations. In other words, the inter-individual variation of the stimulating hormones is much less pronounced than the inter.individual variation of the target cell hormones.

Therefore, it may be evident, that primary hypo hormonal states are most easily detected by the assay of the hormone that stimulates the target organ. Thus, to diagnose primary hypothyroidism, the plasma level of TSH should be measured, while in primary mypogonadism (as for example in postmenopausal women), the determination of the concentrations of LH and FSH should be considered. The degree of increase of the concentrations depends on the extent of the reduction of the target cell hormone concentrations. (However, one must be aware of one of the properties of negative feedback systems, e.g. the possibility of stimulating the production of the target cell hormone to (near) normal concentrations by raising the concentration of the stimulating hormone. Such situations occur only rarely.)

In slight degrees of hypothyroidism (subclinical hypothyroidism) the TSH is only slightly elevated, whereas in such cases $\mathrm{T}_{4}$ and $\mathrm{f}_{-} \mathrm{T}_{4}$ are still within the reference values of the laboratory. Biochemically however, such patients must be considered hypothyroid, especially also since the TSH response to TRH is elevated as well (2). In conclusion, the inter-individulal differences of TSH-concentration in normals and hypothyroid patients are less than those of $\mathrm{T}_{4}$ or $f-\mathrm{T}_{4}$. Since the numerical changes in TSH concentration are considerably more pronounced than the corresponding changes in thyroid hormone concentrations, undoubtedly the assessment of TSH concentration must be preferred to determinations of $T_{4}, f_{-} T_{4}$ and $T_{3}$ in screening for primary hypothyroidism.

\subsubsection{Primary hyper hormonal states.}

Primary hyper hormonal states are characterized by target cell hormone concentrations in blood above normal. Again, there is a large overlap between the distributions of the concentrations of the target cell hormones in normal individuals and in patients in a hyper hormonal status. In primary forms of this condition, in which the target organ is functioning autonomously, the negative feedback mechanism of the pituitary target cell axis will inhibit the production and secretion of the stimulating hormone, and consequently, the concentration of the stimulating hormones will be unmeasurable with conventional techniques $(3)$. 
Biochemically, hyperthyroidism is characterized by high to very high levels of thyroid homones ( $T_{4}$ and/or $T_{3}$, figure 1.3).

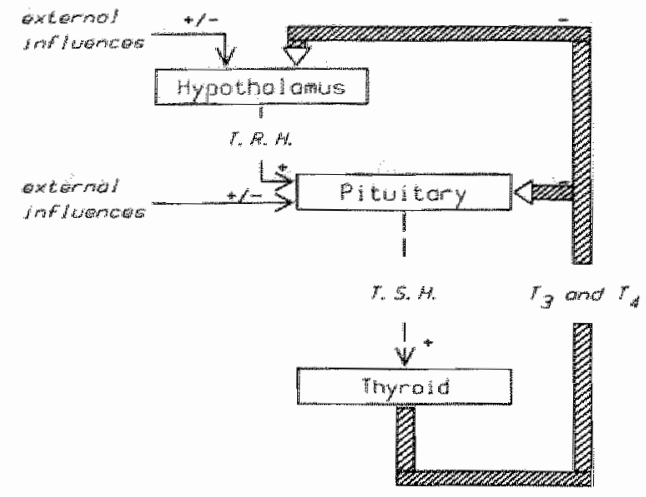

Figure 1.3: Disturbances of teedback mechanism in primary hyperthyroidismt.

Depending on the methodology of the TSH assay, TSH concentrations are found to be (very) low or normal (4). Most commercially available RIA assays are not sensitive enough to distinguish non-zero concentrations of stimulating hormone from zero. Consequently, in patients with primary hyperthyroidism, a most any value for plasma TSH concentrations between zero and $8 \mathrm{mU} / \mathrm{l}$ can be found, while the upper level of the normal range in most RIA assays is about 5 to $8 \mathrm{mU} / \mathrm{I}$. In contrast, the reference values for plasma TSH, estimated by modern two-step IRMA or IRMA-like methods, are measured to be between 0.2 and $3.5 \mathrm{mU} / \mathrm{l}$. In primary hyperthyroidism, plasma TSH concentrations are below the lower limit of normal and often even below the detection limit of the assay. In contrast to primary hyperthyroidism, which occurs frequently, primary autonomous function of the gonads, both the ovaries and the testes, is very rare except for some neoplastic conditions. In fact, in patients with a primary ovarian estrogen producing tumour, e.g. a granulosa cell tumour, extremely suppressed FSH levels can be found. Furthermore, the concentrations of the gonadotrophins are suppressed in patients receiving estrogen therapy.

\subsection{Secondary and tertiary dysregulation of hormonal states.}

\subsubsection{Secondary and tertiary hypo hormonal states.}

As stated above, in secondary hypo hormonal states, the target cell hormone concentration in blood is decreased due to deficiency of stimulating hormone from the pituitary. In this form the defect is located within the pituitary which is unable to produce and/or to secrete the stimulating hormone. Stimulation with the appropriate hypo-thalamic releasing factor (thyrotrophin releasing hormone, TRH or gonadotrophin releasing hormone, Gn-RH) is not able to increase sufficiently the concentration of the stimulating hormone by the pituitary $(5,6)$. The target organ, however, has retained production capacity. This can be demonstrated by injection of the stimulating hormone (TSH, LH or 
FSH), upon which the target organ will produce the appropriate amount of homones (thyroid hormones, sex steroids). In the tertiary form, the concentration of the stimulating hormone is also decreased, but in this case the defect is located in the thypothalamus or even in higher cerebral centres. Since the pituitary has conserved normal capacity to produce and secrete the releasing factor, stimulation with releasing factor will result in a significant rise of the stimulating hormone concentration $(7)$.

However, this response will be below the normal response limits if the state of deficiency existed for a longer period, due to the disappearance or desensitization of the pituitary receptors for the releasing hormone.

In secondary hypothyroidism $T_{4}, f-T_{4}$ and sometimes $T_{3}$ concentrations are below normal (figure 1.4a). In the completely deficient form, plasma TSH levels will not be measurable. but in general practice TSH may have any value between zero and the upper level of normal. Stimulation with TRH shows no response or a greatly diminished TSH response. Since TSH concentrations will be in the lower range, sensitivity of the assay must be high enough to distinguish the differences in TSH after TRH injection. Conventionally RIA based assays of TSH are not sensitive enough for detection of these small differences. Secondary forms of hypogonadism are rare (e.g.Kallmann's syndrome). In these cases, there is a lack of the production and secretion of the sex steroids due to insufficient stimulation of the gonads by LH and FSH (figure 1.4b).

In some clinical conditions, however, it is important to know whether or not there is any production of stimulating hormone. For example, in precocious puberty treatment may be different regarding if the disease is originating from the pituitary or from the gonads (8), Furthermore, since neoplastic compression of the pituitary manifests itself firstly by decreasing the production rates of $\mathrm{LH}$ and FSH (rather than those of TSH or ACTH), it is important that low concentrations of these hormones can be measured with great precision.
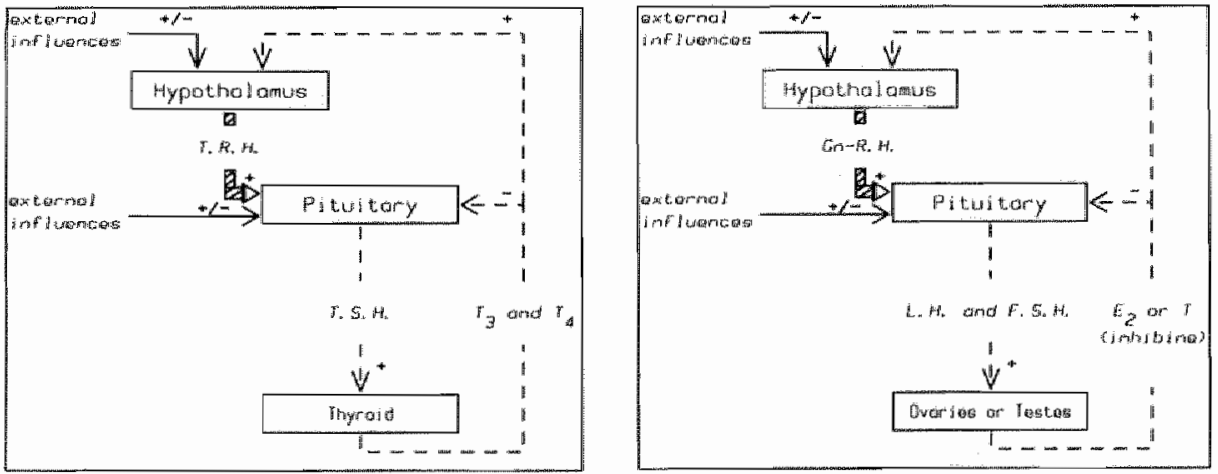

Figure 1.4: Disturbances of feedback mechanism a: in secondary hypothyroidism.

b: in hypogonadotropic hypogonadism. 


\subsubsection{Secondary and tertiary hyper hormonal states.}

Pituitary acenomala are relatively rare, bul may be responsible for hypersecretion of stimulating hormones, resulting in overstimulation of the targel cells. Pituitary resistance to the peripheral hommenes might be another cause of hyper homonal states, leading lo dysunction or even abolishment of the negative feedback mechanism.

Finally, hyperstimulation of the pituitary by releasing factors from the hypothalamus must also be considered as a possiblity in hyper hormonal states (9).

Hypersecretion of TSH is rare, but is sometimes the cause of hyperthyroidism. It is often associated with rypersecretion of growth homone and/or prolactin (10). The TSH concentration does not increase upon stimulation with TRH, nor does it decrease after suppletion with thyroid homones.

Secondary or tertary hypersecrebion of gonadotrophins is also rare. In chidren it may lead to premature puberty, most probably caused by hypothalamic origin(11). In polycystic ovarian disease (PCOD), moderate oversecretion of $\mathrm{LH}$, accompanied by normal or decreased concentrations of FSH is observed. Infertility due to PCOD cam be treated by suppression with an Gn-RH analogue and subsequent stimulation with exogenous FSH and LH (68). This indicates a possible role of the pituitary in the disease. However, the ovaries may be involved also in the pathogenesis of PCOD. In fact, since polycystic ovaries are found in many diseases such as Cushing:s syndrome, obesity, adrenal hyperplasia, etc. other mechanisms may be operative in PCOD. At this moment, for example, little is known about the behaviour of inhibin (a hormone produced by the ovaries) in PCOD with regard to its normal suppressive action on FSH secretion.

\subsection{Sensitive Measurements of Stimulating Hormones.}

From the previous paragraphs, it may be obvious that in normal physiology only narrow concentration ranges of the stimulating hormones have to be expected. In practice this has been proven true: the physiological range of TSH is found to be $0.4-3.5[\mathrm{mU} / \mathrm{l} / \mathrm{l}$ (12) and some authors recommend an even narrower range of $0.2-2.5[\mathrm{mU} / \mathrm{ll}$ (13). It is impotiant to note that the reference range of TSH tends to decrease with age (14,15). For LH, the reference range is given as 2 - 15 [U/l] in men and premenopausal women during the tollicular of luteal phases, FSH normally is present in plasma concentrations of $2-12$ [U/I, equally in men and premenopausal women during the follicular or luteal phases. Above normal blood levels of stimulating hormones indicate below nomal concentrations of the target cell hormones in primany dystunction and hyperstimulation in secondary or tertiary forms of disease. Even with conventional PIA methods such increased values of the stimulating hormone are generally not difficult to detect. Only in cases when the analytical sensitivity of the assays equals or even exceeds the physiological upper limits of normal, do problems arise. Especially, this may be dangerous in the establistmment of the secondary and tertiary forms of disease, since these may be characterized by only slight increases in the concentrations of the stimulating hormone. Values below normal are found in patients with increased concentrations of target cell hormones but are rarely noted in patients with pituitary or hypothalamic disease. For a long time the detection of such low values has been impossible in routine clinical 
chemistry. Generally, with most conventional RIA assays only the upper limits of the reference values could be established because of the lack of sensitivity of the assays. in routine clinical practice, this situation produced several major problems, which were difficult to recognize. These included:

- distinction of hyperthyroid from euthyroid patients was impossible on the basis of TSH levels:

- slight increases in plasma TSH concentration due to subcilinical states of hypothyroidism were generally considered to be non significant;

- the decreasing concentration of TSH in old age was not recognized;

- true (hypogonadotropic) hypogonadal states could not be detected by conventional RIA-based LH and FSH assays;

- the detection of the onset of puberty using the demonstration of periodic (nocturnal) release of gonadotrophins was difficult due to the lack of sensitivity of the assays:

- the pulsatile secretion of neither TSH nor (to a minor extent) LH could be studied. Variations could not be correlated with clinical conditions.

In the above paragraphs, it has been deduced from a theoretical point of view that the recognition of low values for the concentrations of stimulating hormones is important in clinical diagnosis and for physiological studies.

In the next paragraph the advantages of sensitive measurements of two of the pituitary hormones, i.e. TSH and LH will be discussed.

\subsubsection{Sensitive estimation of TSH concentrations.}

Until 1985 no routine methods were available for measuring low concentrations of TSH. However, the importance of determining Iow TSH concentrations had already been demonstrated in 1969 by Adams et al.(16), in 1979 by Wehmann et al.(17) and in 1980 by Spencer et al.(18). The developed RIAS of TSH reached a high sensitivity by extraction procedures of TSH from the serum, long incubation periods and delayed addition of freshly purified tracer. Up to the late seventies, the most sensitive analytical assay for TSH was the cytochemical method of Bitensky (19). However, this method was still more labourious and time consuming then the FIA methods. In the early eighties, Ekins (20) proposed a theoretical basis for the development of immunoassays of much higher sensitivity. An important breakthrough was the development of production methods for highly specific monoclonal antibodies (21).

In the early 1984, monoclonal antibodies raised against highly characterized epitopes of the TSH molecule became available. The combination of one antibody raised towards the beta subunit of the molecule with a second monoclonal antibody directed to the transition of the alpha to the beta subunit in a so called sandwich assay yielded a highly specific. highly sensitive assay for TSH $(12,22)$. Due to the sandwich character of the assay short incubation times are now sufficient and generally it is possible to perform the whole assay (containing 30-40 patient samples in duplicate) within five hours. Some methodologies even permit shorter incubation times (23). 
The first assay commercially available was the assay of Boots-Cellech (Slough, U.K.) (24). This method uses two monoclonal antibodies that are direcled against epitopes as described above. The separation of the bound and the free fractions is based on the different behaviour of the labelled antibody and the labelled antibody-antigen-second antibody complex in a sucrose gradient solution. This separation method has been proven to be highly efficient (25).

The clinical advantages have been widely reported (26-66). Various authors demonstrated the differences between the concentrations in hyperthyroid and euthyroid patients.

Numerous studies have been dedicaled to the recognition of thyroid disease in severely ill patients: The recognition of thyroid disease in patients using amiodarone, impossible with old types of assays, became possible with the new TSH method. The study of small variations of the TSH concentration in response to minor changes in the thyroid hormones (this thesis chapter 3.1) or to changes in metabolism (this thesis chapter 3.2) would not have yielded significant results if conventional types of TSH assays had been used. Studies of pulsatility of TSH and especially changes in pulse frequency and/or pulse amplitude under different conditions (this thesis, chapter 5) are impossible or severely hampered with conventional RIA type assays, since all peak detection algorhythms use the assay variation to discriminate between peaks and noise within time series.

\subsubsection{Sensitive estimation of luteinizing hormone.}

Based on the success of the highly specific, highily sensitive assay of TSH, manulacturers searched for similar types of assays for other protein hormones and especially for pituitary hormones. One of the first commercially available assays for $\mathrm{LH}_{\text {, based on the }}$ monoclonal sandwich technique was from the Boots-Celltech Company too. This manufacturer also produced an assay for the determination of FSH based on the same principles.

The assay for LH has a precision performance better than $10 \%$ in the range of $1-200 \mathrm{U} / 1$ and even below $5 \%$ in the range of 2 to $150 \mathrm{U} / \mathrm{l}$. The sensitivity of the assay is $0.4 \mathrm{U} /$, which is about ten times better than the previous routine method using conventional techniques.

Despite the improved analytical sensitivity and specificity, no advantages (compared to RIA based methods) in clinical praclice have been published to date.

Until now the sensitive measurements of LH and FSH in plasma have not become routine in clinical chemistry. At least two reasons can be given for this.

Firstly, there is considerable doubt about the specificity of the assays. In fact, up to five different forms of the LH molecule have been reported (67), each of them having different behaviour in electrophoretic systems. Manufacturers of sensitive $L H$ assays are unable to tell if their assays recognize one or more iso-hormones better than the others.

Furthermore, the exact biological activity and therefore the clinical importance of each of these iso-hormones is unknown. Only limited information is available about the occurrence of the various iso-hormones in different (patho)physiological states in men and women. Secondly, clinicians are not aware of the advantages of sensitive gonadotrophin assays. This is quite understandable since only a limited number of patients wili benefit from the 
improved sensitivity. Therefore, insufficient patient data are avalable to demonstrate the improvement of nosologic sensitivity and specificity of the assays previously mentioned However, in studies of episodic (or pulsatile) homone secretion, small changes in plasma hormone concentration might be of physiological value. Hence, in this case it is very important to have low coefficients of variation of the hormone estimations. All peak detection programs are based on the recognition of differences between acrophase and nadir greater than a number times the standard deviation of the assay. By improving the coefficient of variation, the signal-to-noise ratio is also improved and, in consequence, the detection of peaks is less aleatory. In particular, the number of false negative errors will diminish. Due to the increased specificity of the assays, type 1 errors (false positive errors) will occur less frequently.

In conclusion, only a small number of investigations dealing with highly sensitive TSH and/or gonadotrophin assays have been published to date. This seriousily hampers our understanding of the underlying mechanism of changes in plasma hormone concentrations in different (patho)physiological conditions. Especially in the grey region between evident pathology and slight disease, the use of sensitive and specific methods may be beneficial.

Therefore, the objectives of the present study were:

- to demonstrate the superiority of sensitive TSH measurements in the study of slight changes in the thyroid hormone status in some clinical conditions;

- to demonstrate the necessity of the use of specific and sensitive assays in the study of episodic and pulsatile secretion of the pituitary hormones. 


\section{References}

1. Distelano d.J., Stubberud A.R. Whilams I. A.: Theory and Problems of reedback and Control Systems. New York: Schaum Pubilithing (1967).

2.Evered D.C., Ormston B.J., Smith P.A. Hall R. Bird T.: Grades of Mypothyroidism. Br Med.u. (1973) i: 657.662

3:Morley d E. Neuroendochine control of thyrotropin secretion. Endocr. Rev.(1981) 2;396 436

4 Anonymus: Assessment of Cinical Technologies: Test for abnormal Thyoio Function. Document by a Who group of experts Lab/85.2 (1985)

5.Faglia G., Beck Peccoz P. Ferrari C., Ambrosi B., Spada A., TravaglinBi P., Paraccihi S.: Plasma thyrotropin response to T.R.H. in patents with pituitay and hypothalamic

disorders.J.Clin Endocrinol. Metab.(1973) 37;595-601

8. Snyder P.J. Rudenstein R.S., Gardner D.F. Pohman J.G. Repetitive infusion of gonadotropin- releasing homone distinguishes hypothakamic from pitutany hypogonadism. J.Clin. Endocrinol.Merab. (1979) $48,864,868$

7 Scanlon MF, Rees Smith B.,Hall R.: Thyold stimulating homone. Neuroregulation and clinical applications. Part 2 Clin.Sci.Mol.Med.(1978) 55:2;129-138

8:Delemarrewari de Waal H.A. Brande J.L.van den, Schoemaker J. Frolonged pulsatile administration of uteinizing hornone releasing homone in prepubertal chibren: Diagnostic and physiologic aspects.

J.Clin. Endocrinol. Metalo (1985) 61; 859-867

9:Daughaday W.H.: The Anterior Pituitary. In: Williams Textbook of Endocrinology Saunders Company (1985) pp 568-613

10.Weintraub B.D., Gershengom M.C., Kourides L.A. Fein H.: Inappropriate secretion of thyroid simulating hormone. Ann Intern. Med.(1981) 95;339-351

11:Kenny F.M., Midgley A.IP, , Jatte R.B., Garces L.Y., Vazques A., Taylar F.H., Saglya C.:

Radioimmunoassayable serum LH and FSH in girls with sexual precocity, premature thelarohe and aorenarche. J.Clin Endocrinol.Metab.(1969) 29;1272-1275

12: Seth J. "Kellett H.A., Caldwell G., Sweeting V.M., Beckett G.d., Gow S.M., Toft A.D.: A sensitwe immunoradiometric assay for serum thyroid stimulating hormone: a replacement for the thyrotrophin releasing hormone test? Br.Med.J.(1984) 289;1334-1336

13.Kreutzer H.J.H., Tertoolen J.F.W., Thijssen J.H.H., Kinderen P.J. der, Koppeschaar H.P.F.: Anahytical evaluation of four sensitive assays af thyrotropin, including effects of variations in patien sampling.

Clin Chem.(1986) 32;2085-2090

14:Snijder P.J., Utiger R.D.: Response to Thyrotropin Releasing Hormone (TRH) in Normal Man.

J.Clin.Endacrinol.Metab.(1972) 34;380-385

15:Erfurth E.M., Norden N.E., Hedner P. Nilsson A. Lars E. K.: Normal Reference interval for Thyrotropin

Response to Thyroliberin: Dependence on Age, Sex, Free Thyroxin Index and Basal Concentrations of

Thyratropin. Clin Chem.(1984) 30/2;196-199

16:Adam D. O. Kennedy T.H. Purves H.D.: Comparison of the thyroid stimulating homone content of serum from thyrotoxic and esthyroid people. J.Clin. Endocrinol Metab.(1969) 29;900-903

17:Wehman A.E. Pubenstein H.A., Nisula B.C.: A sensitwe convenient radioimmunoassay procedure which demonstrates that serum $\mathrm{h}$ rSH is suppressed below the nomal range in thyrotoxic patients.

Endoorinol.Res.Commun.(1979) 6;249-245

18:Spencer C.A. Nicolofl J.T: Improved radiommunosssay for human TSH. Clin.Chim. Acta(1980)

$108 ; 415-424$

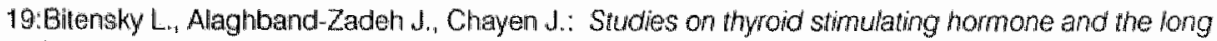
acting thytoid stimulating hormone. Clin. Endocrinol.(1974) 3,363-374

20. Ekins R.P.: Towards immunaassays of greater sensitivity, specificity and speed: An Overview. in: Monoclonal antibodies and developments in Immunoassay. (Ed.A. Albertini and P.P. Ekins)

Elsevier/North Holland Biomedical Press, Amsterdam (1981);3-21

21:Kohler $\mathrm{G}_{\text {a }}$ Milstein $\mathrm{C}$ : Contiruous culture of fused cells secreting antibody of predefined specificity.

Nature (1975) 256;445-497

22: Weeks I., Sturgess M. Siddle K., Jones M.K. Woodhead J.S.: A High Sensitivity

trumunochemiluminometric Assay for Human Thyrotrophin Clin. Endocrinol.(1984) 20,489-495 
23: Carayon P. Martino E. Bartalena L. Grasso L., Mammoli C. Costagliola S. Pinchera A.: Chinical usefuness and bimtations of serum thyrotropin measurement by utrasensitwe method: Comparison of the kits. Hormone Res. (1987) 26(1-4):105-117

24.Alexander W.D. Kerr D.J., Ferguson M.M.: First-Line Test of Thyroid Function. The Lancet (1984), il $647-648$

25:Wright J.F., Hunter W.M. The sucrose layering sepanation: A non centrifugation system. Im:

Immuncassays for Clinical Chemistry (Eds. W.M.Hunter and J.E.T. Corrie) Churchill Livingstone, Edinburgh (1983): $170-177$

26:Allen K.R., Watson D.: First-line test of Thyrold Function. The Lancel (1984); ii 923.

27:Ker D.d. Alexander W.D.: Is the TRH tesl usually unnecessian?? The Lancet (1984)" if 1161-1162

28: Caldwell G., Toft A.D.: First line test of Thyroid function. The Lancet (1984); ii 1162

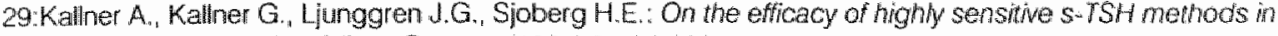
the diagnosis of hyperthyroidism. Opmear (1984) 29:98-1101

30. Semple C.G., Slater S.D. Reid A.M., Gten A.C.A.: A sensitive inmunoradiometric assay for senum thyroid stimulating hormore. Brit.Med. J, (1985) 290;69-70

31: Wood W. G., Waller D. Hantke U.: An evaluation of Six Solid phase hyrotropin (TSH) kits.

J.Clin.Chem.Clin.Biochem.(1985) 23;461-471

32:Wehmann R.E. Halle A.B.: Chinical Uthity of Thyrotropin assays. The responsibiliy of the clinical chemist.

Clin. Chem (1985) 31;329

33:Watanabe Y., Amino N., Tamaki H., Aozasa M., Tachi J., Endo Y. Miyai K... Highty sensitive

immunoenzymometric assay for human thyrovopin. Clin.Chem.(1985) 31;634-636

34:Plannenstiel P. Panitz: Erste Ertahrungen mit einem hochsensitwen wmumometrischen TSH-Assay zur

Diagnostik won Schilddrusenfunktionsstorungen. Der Nuklearmediziner (1985) 2;185-189

35:Gow S.M., Kellett H.A., Seth J., Sweeling V.M., Tolt A.D., Beckelt G.a., Limdations of new thyroid function tests in pregnancy.Clin.Chim.Acta (1985), 152;325-333

36: Joseph K.: Erstiagnostik und Verlaufskontrolle bei Schilddrusen funcionsstorungen mit der

immunadiometrischen TSH-bestimmung. NucCompact (1985) 16:240-254

37:Klingler W. Ball P.: Die Bestimmung ces Thyreotropin (TSH) im Serum met einer sensitiven

immunoradiometrischen Methode. Artztl.Lab.(1985) 31,219-223

38:Bayer M.F., Kriss J.P. McDougall I.R.: Clinical Experience with sensitwe thyrotropin measurements:

diagnostic and therapeutic implications. J. Nuc.Med.(1985) 26,1248-1256

39:Mardell R.J.. Ganten T.R.: Thyroid Test Strategy. The Lancet (1985); ii 1455

40:Kirkegaard C. Bregengrad C. Feldt-Rasmussen E., Bech K. Faber d.: Thyroid Test Strategy. The Lancel (1985); ii 1455

41:Hoermann A., Saller B. Moser E. Mann K.: Thyroid Test Strategy. The Lancel (1985); ii 1455-1456 42:Beck-Peccoz P., Piscitelli G. Medri G., Ballabio M, Faglia G. Thyroid Test Strategy. The Lancet (1985); ii 1456

43:McBride J.H. Thibeault R.V., Rodgerson D. O.: Thyrotropin as measured by a sensitwe immunoradiometric assay. Clin.Chem.(1985) 31;1865-1867

44:Clark P.MS. Price C.P.: Enzyme-amplified immunoassays: a new uirasensitive assay of thyrotropin evaluated.Clin.Chem.(1986) 32,88-92

45:Gow S.M., Elder A., Caldwell G. Belt G. Setth J, Sweeting V.M., Toll A.D., Beckell G. J.: An improved approach to thyroid function testing in patients with non-thyroidal iness. Clin. Chim. Acta (1986) 158,49458

46: Rosenteld L., Blum M. Immunoradionetric assay for thyratropin should replace the FlA method in

cinical laboratory. Clin. Chem.(1986) 32;232-233

47 :Lamberg B.A., Heleniss $\mathbb{T}$., Liewendaht K.: Assessment of thyroxine suppression in thyroid carcinoma patients with a sensitive immunoradiometric TSH assay. Clin. Endocrinol. (1986), 25;259-263

48:Hopton M.P. Harrop J.S.: Immunoradiometric ASsay of thyotropin as a "first ine thyroid function test in the routine laboratory. Clin. Chem. (1986) 32; 691-693

49.Bernulz C., Horn K.: Egebnisse der gemeinsamen Erprobung eines Enzymimmunoassays fur

Thyrotropin (TSH).J.Clin.Chem.Clin.Biochem.(1986) 24;659-666

50:Wiersinga W.M.: The value of sensitwe TSH measurements in clinical practice. J.Endocrinol linvest.(1986) $9 / 4 ; 67-76$

51:Sheppard M.C. Black E.G.: Clinical application of a sensirwe non isotopic immunometric assay of thyrotropin. Clin.Chem.(1987) 33;179-181 
52: Honnovwessen D, skov L., Faber J.; Immunoradiometric assay of senum thyroid stmulating hormone as a first line test in a mbed hospital population, Clin. Chim.Acta (1987) 168; $13-17$

53:Caldwell G. Gow S.M. Sweeting W.M. Beckett G.u. Seth J., Toft A.D.: Value and limitations of a highy sensituve immunoradiometric assay for thyrotropin in the study of thyrotroph function. Clin. Chem.(1987)

$33 ; 303-305$

5A4:Davis JR.E. Black E G. Sheppard M.C.: Evaluation of a sensitue chemiluminescent assay for TSH in the Jollow up of treated thyrotoxicosis. Clin. Endoctind,(1987) 27,563-570

55:Piketly M.L. "Talbot J.N., Askienazy S., Milhaud G." Clinical significance of a low concentration of thyrotropin: fwe immunometric "kit assays compared.Clin. Chem.(1987) 33;1237-1241

56: Marsteh S.: Caution against spuriously increased thyrotropin watues as determined by two site immunoradiometric assayst. Clin Chem. (1987) 33,1290-1291

57:Kloe G. .. Hay l.D. Assessment of sensitve thyrotropin assays for an expanded role in thyroid function testing: proposed criteria for analytic performance and clinical utility. J. Clin. Endocrinol.Metab.(1987) $64: 461-471$

58: Wheatley T., Clak P.M.S., Clark J.D.A., Raggaft P.R., Edwards O.M.: Thyroid stimulating hormone measurement by ar ultrasensitwe assay during thyroxine replacement; comparison with other test of thyroid function. Ann.Clin Biochem.(1987) 24;614.619

59:Butler $d$, Cundy T.: Serum thyrotrophin in patients with desiructive pitutary lesions assessed by a sensitive immunoradiometric assay. Ann, Clin. Biochem.(1987) 24,620-624

60:Evans P.J. Woodhead J.S. Weeks I., Scanton M.F.; Circulating TSH levels measured with an immunochemiluminometric assay in patients taking drugs interfering with biochemical thyroid status. Clin Endocrinol.(1987) 26:717-721

61: Mudde A.H., Bastiaanse A.d., Jonkers H.: TSH-HRMA als eerste diagnosticum bij vermoeden van hypenthyreoidie. N.T.V.G.(1987) 1.31;2364-2368

62 Krenning E.P., Docter A. Visser T.J., Vos R.A., Toor H. van,Hennemann G.: Sensitwe serum TSH assay.

The initial ciagnostic tool in evaluation of thyroid function.JDR (1987) 12;271-277

63: Evans M.C.: Ten commercial kits compared for assay of thyratropin in the normal and thyrotoxic range.

Clin.Chem.(1988) 34;123-127

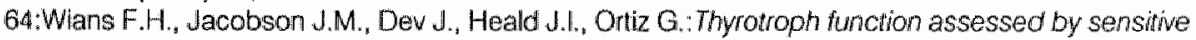
measurement of thyrotropin with three immunoradiometric assay kits: avalytical evaluation and comparison with the thyroliberin stimulation test. Clin. Chem,(1988) 34;568-575

65:Thonnart B. Messian O. Colas Linhart N., Bok B.: Ten highly sensitive thyrotropir assays compared by receiver operating characteristic curves analysis: resulis of a prospective multicenter study.

Clin.Chem.(1988) 34;691-695

66:Toft A.D., Seth J.: Sensitive thyrotrophin assays: excellent when properly used. Brit. Med.4.(1988) 38;62 67:Ginkel L.A. Loeber J.G.: Heterogeneity of human ktropin. Detection and identification of alpha-and beta-Subunits. Acta Endocrinol. (1985) 110;182-192

68:Hamilton $C_{\text {. }}$. Heineman Mud., Menheere P. Evers $H$,: Comparison of the hormonal responses in patients with potycystic ovarian disease and normal ovulating women following a oministration of an LHRH agonist. In: Rolland P., Heineman M.J., Naaktgeboren N., Schoemaker \&., Vemer H., Wilemsen W.N.P. (Edilors): Neuro-endocrinology of reproduction. Excerpla Medica, Amsterdam (1987):pp285-293 69:Ying S:A: Inhibins, activins and folliculostatins: Gonadal proteins modulating the secretion of follicle stimulating hormone Endocrine Rev.(1988) 9,267-293 


\section{Chapter 2}

\section{Sensitive Estimations of Glycoprotein Concentrations.}

\subsection{General Principles of Immunoassays.}

By classical chemical analyses analytes can be estimated in the mmol/ and sometimes even in the $\mu \mathrm{mol} / \mathrm{l}$ range. Specificities and sensitivities in general are good enough for the determination of common substrates in clinical chemistry like glucose, urea and creatinine. The specificity of the reactions may be greatly enhanced by the use of enzymes transforming the substrate to be analyzed to a reaction product, while this reaction or a simultaneously occurring side-reaction is observed.

However, the sensitivity of these methods, even if enhanced by instrumental procedures, is not sufficient for the determination of many important hormones. This is even more true for the regulators of hormone concentrations, which are present in plasma in even lower concentrations. Therefore, the classical chemical methods are quite insufficient for the measurement of their concentrations. For example, no classical method is known for the determination of thyroxine in human plasma. For this reason, clinical chemists used the PBI (protein bound iodine) assay or the somewhat more specific assay of hormonal bound iodine for the assessment of thyroid function. It is obvious that the development of thyroidology would have been considerably delayed if the specific determination of thyroxine and triiodothyronine was still impossible.

The introduction of radioimmunoassay $(\mathrm{R} \mid \mathrm{A})$ in the late lifties really proved to be a break-through for the endocrinologist. It has been the starting point for the development of assays with very high specificity and sensitivity in and outside medical science. The high specificity was the result of the use of specific antisera (polyclonal antibodies). The recently introduced monoclonal antibodies show even higher, although not always desired specificity. (For example in the assay of tumourmarkers too high specificity will result in a too low sensitivity.)

The high sensitivity resulted from the use of radioactive labels, since the detection of radioactivity is extremely sensitive with modern instrumental techniques. However, the limits of sensitivity by the use of radioactive tracers seem to be reached. Alternative tracers are in development and some of them, especially fluorescent and chemiluminescent labels seem very promising.

\subsection{Ligand Receptor Assays.}

All possible configurations of immunoassays are in general described by the term "ligand-receptor-assay". All other names like for example radioimmunoassay, saturation analyses, displacement analyses, immunoradiometric assay or enzymimmunoassay, accentuate one or more properties of a certain particular procedure not present in the others. 

All ligand receptor assays are characterized by four important features
a: one or more highly specific receptor(s):
b: a labelled ligand preferentialy with a high specitic activity:
c: an unlabelled ligand in very pure form to be used as standard:
d: a separation method to perform a complete separation of the bound and the free fraction.

\section{Each of these items will be shorty reviewed.}

\section{a: the receptor}

The receptor as used within the ligand-receptor assay is a substance of protein origin that specifically binds the ligand. Normally the receptor is an antibody, but other binders such as membrane receptors, nuclear receptors and plasma transporting proteins have equally specific binding properties and so can also be used in ligand-receptor assays

b. the ligand

The igand must be avallable in a pure form for three reasons: firstly, the ligand is needed to provoke the antibody response within the animal used to raise the antibody. For this purpose it is essential that the ligand is free of any impurity that might cause cross reaction in vivo. Secondly, the ligand in pure form is needed to calibrate the assay and finally the ligand is used to be labelled itself.

c: the labelled ligand

The label is used for the measurement of the immunochemical reaction. The first labels used were ${ }^{131} \|$ (later ${ }^{125}$ ) for the labelling of large molecules and tritium and carbon-14 for small molecules like steroids and drugs. lodine can be easily incorporated in aromatic rings like those of tyrosine and histidine. if these amino acids are present within the analyte molecule the labelling is no problem. Small molecules can not be labelled without the introduction in the molecule of a bridge to an aromatic group. Fortunately, in recent year's the techniques of derivatization have been much improved and actually it is possible to label smal molecules llke steroids. with very large tracers like enzymes. Other currently used labels are composed ffluorescent or chemiluminescent molecules or contain rare metal particies like gold or silver.

d: the separation method

The bound fraction has to be separated from the free fraction in a quantitative way. The ratio between the counts present in both fractions is the response parameter of the immunochernical reaction. The separation step often is based on physico-chemical differences belween the bound and the unbound ligand. Common methods include precipitation by second antibody with or without poly-ethylene-glyool (PEG), absorption by charcoal or sephadex and methods based on solid phase techniques like coated lubes. coated beads, elc.

The work described in this thesis has been pelformed mostly by immunoradiometric assays (IPMA). Enhanced sensitivity and specificity in comparison to former radioimmunoassays (RIA) were the basis of new applications in routine clinical analysis and research. Both these immunochemical techniques are reviewed shortly and a description is given of the antibodies used in these type of assays. 


\subsubsection{Radioimmunoassays (RIA)}

In conventional RIA-procedures as developed and described by Berson and Yalow (1) and by Ekins (2) a limited amount of receptor reacts with the analyte and a limited amount of radiolabelled analyte. A separation step is needed to separate completely the unbound ligand from the ligand bound to the receptor. Calibration of the assay is performed by the use of highly purified analyte in known concentrations.

Schematically the RIA can be represented as:

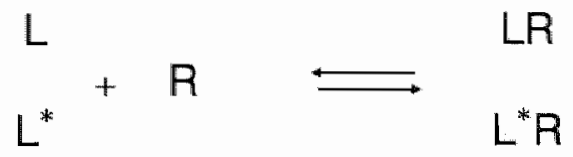

in which:

L: $\quad$ the analyte or ligand to be determined

$L^{*}: \quad$ the labelled analyte or labelled ligand

$R$ the receptor or antibody

LR: $\quad$ the unlabelled analyte-antibody complex

$L^{*} R$ : the labelled analyte-antibody complex

The calibration curve in a RIA has a typically sigmoidal form (figure 2.1). It is important to note that the response parameter $(B / T)$ has its highest value in the absence of unlabelled ligand; the amount of tracer bound $\left(L^{*} R\right)$ is indicated as $B$, while the total amount of tracer $\left(L^{*}+L^{*} R\right)$ is indicated as $T$. The more analyte is added, the less activity is found in the bound fraction. The shape of the curve is markedly influenced by relative concentrations of antibody and labelled ligand. Minor changes can be necessary to have the steepest part of the curve correspond to the clinically most interesting part of the dynamic range of the assay. Rigid quality control procedures are necessary in order to maintain the selected region of highest precision and accuracy within acceptable limits.

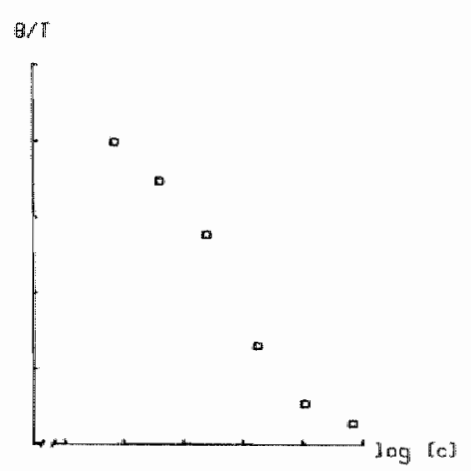

Figure 21:

Typical imrnunoassay calibration curve.

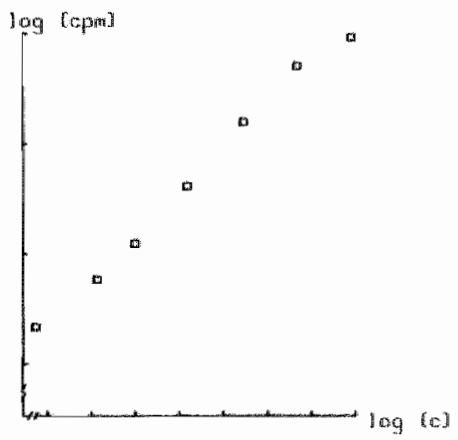

Figure 2.2:

Typical callibation cumve of an immunometric assay. 


\subsubsection{Immunoradiometric assay (IRMA).}

In contrast to RIA, the tracer in this type of assay is not bound to the ligand, but to the receptor molecule.

The schematic presentation of the IRMA is as follows:

$$
\begin{aligned}
\mathrm{L}+\mathrm{R}_{1}-\mathrm{s} & \longrightarrow \mathrm{L}-\mathrm{R}_{1}-\mathrm{s} \\
\mathrm{L}-\mathrm{R}_{1}-\mathrm{s}+\mathrm{R}_{2}^{*} & \longrightarrow \mathrm{s}-\mathrm{R}_{1}-\mathrm{L}-\mathrm{R}_{2}^{*}
\end{aligned}
$$

In which:

L:

P1-S:

L-R $-\mathrm{S}$ :

A:

$s-R_{1}-L-R_{2}^{*}$

the analyte or ligand to be determined the first antibody coupled covalently to a sollid phase. the analyte or ligand to be determined the labelled second antibody

the sandwich complex of the antigen with both antibodies

Two antibodies are used: one is covalently coupled to a solid phase and the second is labelled. Generally the reactions of antigen and antibodies are thought to be of the equilibrium type. However, actually some immunoradiometric assays are composed of antibodies with such high affinity constants that assays look like end-point reactions and can be completed within $10 \mathrm{~min}$. In the IRMA (as opposite to RIA) the antibodies are present in excess concentrations and it is not the ligand that bears the label but one of the antibodies. The shape of the calibration curve is the inverse of that of RIA (figure 2.2): by increasing concentrations of the analyte the response metameter increases also.

\subsection{Antibodies and their impacts on specificity}

The limited amount of antibody is principally the most important characteristic of a radioimmunoassay. Antigen and labelled antigen have to compete for binding sites on the antibody. Competition is only possible at very high dilutions of the antibody. Thus the antibody is used in a very efficient way and often millions of samples can be analyzed by a single batch of antiserum.

Excess concentrations of antibodies in IRMA necessitates the use of larger quantities of antibody.

For practical reasons until recently, only limited quantities of antibodies could be produced. The reduction of the quantity of antibody to be used in each assay to an absolute minimum was an important condition for long term continuity in the quality of the results. Nowadays, almost unlimited amounts of antibody can be produced by using monoclonal production techniques.

The antibodies used in most RIA's for the estimation of analytes of human origin are raised in animals. Higher animals have a very effective immune system in order to eliminate foreign substances. The immune system is able to recognize minor differences in molecular structure. This is necessary to differentiate between proper and foreign 
subslances both of which might have biologic activily within that animal.

The only condition that must be satisfied for the immune system to provoke an immune response is that the foreign substance has immunogenic properties. Only targe molecules such as proteins and compounds containing large carbohydrate groups have immunogenic properties. Smaller molecules (in general with a molecular weight below 3000 [Daltons]) can be made immunogenic by coupling with carrier molecules like thyroglobulin $(\mathrm{Tg})$ or albumin. Such methods for example are largely used for the production of antibodies against steroids. The antigen is injected subcutaneously in an adjuvant mixture (often Freunds adjuvant: a mixture of antigen in an oil phase activated with killed mycobacteria). If there is a response of the immune system multiple antibodies are produced, directed toward different epitopes of the antigen or hapten-carrier molecule-complex. In fact, the tertiary structure of the sequence of only some (4 to 8) amino acids within the antigen molecule is sufficient to provoke the immune response explaining why within one antigen different regions can have immunogenic properties. The produced antibodies all have different affinities and titers and together they form the antiserum.

Obviously such antiserum can react with the antigen but also with subunits of the antigen or with other substances showing cross reactions. Furthermore, antibodies may have been formed to contaminants that were present in the vaccination preparation since impurities may induce antibodies too. Their influence in the assay can be absent (depending on the presence of such substances in the patient samples) or can be very important (if their structure is closely related to the antigen). For this reason antisera often, although not always need some purification. The purified product, however, still will be composed of numerous antibodies. For this reason such a mixture of anlibodies direcled principally toward one antigen is called a polyclonal antibody or antiserum.

Since 1979 a totally different way of production of antisera developed by Milstein and Koehler is known (3). They developed a cell fusion technique to combine the properties of two related cell-lines, one being a single clone of B-lymphoid cells and the other being a myeloma cell-line.

B-lymphoid cells have the possibility to produce only one antibody, but these cells die off in-vitro after they have been taken from the spleen of a sensitized animal.

Myeloma cells (of rodents) can be cultured in-vitro and it is possiblc to induce the growth of myelomas as tumour in-vivo.

Combination of both types of cells may result in a hybridoma, tetraploid stable cells that have retained the characteristics of the original cells, i.e the possibility of production of only one clone of antibody without dying. (It is beyond the scope of this chapter to discuss the conditions necessary in order to maintain the hybridoma cells actively divide while the myeloma cell and the B-lymphoid cell die.) The produced antibodies can react only with one epitope of the antigen. The specificity of so produced antisera is much enhanced in comparison to conventionally production methods. However, it must be realized that cross reactivity still exists since only part of the molecule is involved in the antigen-antibody reaction. Other related compounds may have the same epitopic site leading to cross reaction. The phenomenon of cross reaction can be reduced dramatically by the simultaneous use of two monoclonal antibodies, the antigen being enclosed between. One monoclonal antibody is directed to an epitope at one sile of the antigen and the second antibody must be directed to another epitope of the antigen in 
such a way that the enclosed part can be lound only in one type of molecule, i.e. the antigen.

\subsubsection{Measurement of bioactivity or of immunoreactivity?}

Since the introduction of immunochemical methods there have been discussions about the relation of bioactivity of the measured compound and its immunoreactivity. In fact, since the in-vivo receptors of the antigen are in general not the same as the antibodies used in-vitro, differences can be expected.

Long before the era of radioimmunoassay, bioassays were known: assays in which the hormone to be measured provoked a response in a biosystem (often in an other species). From the results conclusions are drawn about the in-vivo activity of the hormone in humans. Some examples of these are the mouse hypoglycemia test for insulin (4), the dispersed rat Leydig cells test for the assay of LH (5) and the G6PD stimulation of the cortical tubuli cells in guinea pig for PTH (6).

Bioassays exist in various forms using whole animals, organ preparations and cytochemical assays. Due to their lack in sensitivity and their complexibility, the assays using whole animals and organ preparations have almost completely disappeared. Cytochemical assays, in general, are more sensitive than bioassays and somelimes even more sensitive than (radio)immunoassays, but few are available. Still, they are very complex and not sutable for large scale routine use.

Both the in-vitro immunaassay and the bioassay use the binding of the antigen with a receptor:

- in bioassays the result of the addition of a unknown quantity of antigen is followed by a response of the biosystem. This can be a volume change of the organ or an increase of the metabolic activity of the cell. Changes in cell activity can be detected by the assay of products of cell metabolism like the production of testosterone by ral Leydig cells when stimulated with LH.

- In the immunoassay the result is measured by means of the distribution over the bound and the free fraction of the labelled molecules (either the antigen or one of the antibodies). Thereupon, this ratio can be translated into an antigen concentration using a calibration curve,

A gradual scale for the relation between the bioassay and the immunoassay is published by Bangham (7). In his opinion, each bioassay is an immunoassay, but immunoassays. generally, are not to be contused with bioassays.

The real difference between immunoassays and bioassays is defined by the genetic information that is contained in the mucleus. This information influences the response of the intact cell system. Within the immunoassay no genetic information is present at all and the response of the system obeys only to thermodynamic laws.

Immunoassays are able to recognize all kind of molecules, provided that they are immunogenic. The biological activity of it in humans does not determine whether or not a molecule is immunogenic. Therefore, it is possible to raise antibodies directed towards compounds without any biological activity in-vivo. Compounds that lost their bioactivity in-vivo due to slight modifications of the molecule (biological degradation, hydrolyses, sialysation or carboxylation) still may possess the epitope towards which the antibody was 
directed. In short, bioactivity and immunoreactivity may be different, if the epitopic site on the molecule is not the same as the biological active site: It appears that minor changes in molecular conformation may be enough to the molecule to lose all of its biological activity. in clinical practice this has important consequences. In tact, a number of messenger molecules has easy degradable sites in order to shorten the time of action of the messenger in-vivo. After discharging in circulation, such molecules rapidly lose biological activity by actions of (among others) liver or kidneys but the formed derivative may stay in circulation for a long time. Clinical chemists will only be interested in the active molecule. but most antibodies recognize both the original molecule and the modified molecule. For example, for long time only total immunoreactive PTH could be measured by immunological methods. These assays detected the intact PTH $[1,84]$ molecule but also the preprohormone[-6, 102], the prehormone $[-6,84]$ and the fragments like PTH $[1,34]$. PTH $[44,59]$ and PTH $[39,84]$. Recently, two site sandwich assays have been developed that are specific for the intact molecule PTH $[1,84]$. The measured PTH concentrations are about 100 times lower than that of total immunoreactive PTH. The correlation with diseases proved to be much better. However, with these assays the biologically active subfraction PTH $[1,8]$ is not detected neither.

The pituitary glycoproteins TSH, LH and FSH are composed of a protein core and of branched carbohydrate chains (8). These chains usually terminate with sialic acid. Two non-covalently coupled subunits (called the alpha- and the beta subunit) characterize the protein core. The alpha subunit is present in all above molecules and in hCG. The beta subunit is different. Both the subunits have been demonstrated in plasma, but are essentially devoid of any biological action (as far as our knowledge reaches for the moment). The biological activity is exercised by the intact molecule only. However, this biological activity will greatly depend upon the carbohydrate content of the molecule. Different sialic acid content of the molecules further will result in variations of the physicochemical properties, immunoreactivity, bioactivity, plasma half-life both in-vivo and in-vitro (9). In practice, it will be impossible to obtain the biological active form of the molecule in pure form. There will be a mixture of molecules with tertiary structures all with different biological behaviour. Raising an antibody against such a solution will result in the production of an antiserum, in which some of the antibodies are directed towards epitopes on the intact molecule containing part of the carbohydrate chain and/or one of more sialic acid groups. Although these parts of the antigen molecule probably do not react with the antibody, they may be necessary in order to provide the correct tertiary structure of the antigen molecule in the reaction with the antibody. The selection of only one clone of antibody, as is done in the production techniques of monoclonal antibodies, is essentially identical to the selection of an antibody against an antigen with a well defined biological activity, although this does not imply that this biological behaviour is known in all clinical conditions. Two main problems will result from this procedure: firstly, using such antibody preparation in immunoassay techniques will lead to differences between the immuno logic and biological activity of the antigen. In fact, all the molecules that do not react with the selected antibody are not necessarily free of biological potency. Moreover, it is not impossible that those unreacting antigens have greater biological activities that the antibody specific antigen. Secondly, the latter antigen is not necessarily present in all clinical states. Hypo-, eu- or hyperhormonal states may change the equilibrium between the various molecular forms of the antigen in order to maintain homeostasis 
Hypohormonal states, for example, may favour the presence of antigens with a longer half fife (thus containing more sialic acid), while hyperhormonal states will do the opposite. Consequently, the hormone would have a different conformation in the various pathological states. For TSH, for example, this would result in three torms for the TSH molecule: one in cases of euthyroidism, one in hyperthyroidism and finally one in hypothyroidism. Since the half-lives of the molecule increase going from the hyperthyroidic state through the euthyroidic sitate to the hypothyroidic state, it is possible that all forms originate from one molecule that, secreted by the pituitary is more or less longer exposed to possible transformations due to the action of liver and kidneys.

Thus, theoretically it is possible to have samples showing no immunologic activity, while in the bioassay the same samples are hyper active. In practice there are aiready some examples of an undesired high specificity of an immunoassay coupled with a unacceptable decrease in nosological sensitivity of the assay: too high specificity of the antibody against ACTH may result in non recogrizing of big-ACTH and of parts of the ACTH molecule as these are produced by ectopic ACTH-secreting tumours (10).

Furthermore the increased specificity of a two side monoclonal IRMA for the measurement of thyroglobulin (11) gives a diminished sensitivity in the recognition of recidives of thyroid carcinoma in comparison to a conventional RIA procedure using polyclonal antibodies. To date, there is evidence that even in non-pathological conditions different forms of the same hormones exist: van Ginkel (12) demonstrated 5 components with different properties in electrofocusing separation procedures, all showing $\mathrm{LH}$-activity in in-vitro bioassay.

It is evident that although conventional RIA procedures using polyclonal antibodies, up to now did not show great discrepancies between immunological and biological activity, the increased specificity of the monoclonal antibodies can lead to misinterpretations.

Probably, this is mostly due to the co-existence of different molecular forms and structures of the antigen some of which have biological activity and others that show only immunologic activity. Of course, all kinds of molecular forms will be present in-vivo while in pathological conditions the variance may even be greater.

\subsection{Tracers and their impacts on sensitivity}

Sensitivity can be defined in two fundamental different ways.

The first concept is of Berson and Yalow (13), the pioneers of radioimmunoassay. They consider sensitivity to be equal to the slope of the dose-response curve. Sensitivity thus can be defined on each point of the standard curve. If there is a linear curve the slope and hence, the sensitivity will be the same over the complete dynamic range. However, the usual curves in radioimmunoassay have a non-linear sigmoidal character and consequently, sensitivity depends on the concentration level of the analyte. Sensitivity is the smallest change in the response metameter, that can be measured by a minor change in the ligand concentration.

Sensitivity is also closely related to the least detectable concentration. Furthermore the lower detection limit is an important property of each analysis. For this reason, Ekins (14) definition of sensitivity is entirely different. In his concept sensitivity is defined as the quantity of unlabelled hormone that causes the response metameter to change with a 
magnitude as great as the standard deviation of that response metameter at zero concentration of hormone. By this definition the sensitivity is identical to the lower detection limit.

\subsubsection{Competitive Immunoassays.}

The general equation for the reaction in a competitive immunoassay is:

$$
L+R \quad \rightleftarrows L R
$$

in which:
L:
R:
Ligand or analyte
LR:
Receptor or antibody
Ligand-Receptor-complex

According to Rodbard (15) we suppose the following basic conditions to be satisfied:

- the receptor is present in a homogeneous form;

- the ligand is present in a homogeneous form;

- the state of equilibrium has been reached;

- one molecule of ligand can only react with one molecule of receptor and visa versa (univalency);

- there are no allosteric or cooperative effects in the binding reaction of ligand and receptor:

- there are no differences in the reactions of ligand or labelled ligand with the receptor:

- the separation step can be performed without misclassification errors and without disturbance of the equilibrium.

If all the conditions are satisfied the equilibrium state can be described by classical thermodynamic laws:

$$
\mathrm{K}_{\alpha}=\frac{[\mathrm{LR}]}{[\mathrm{L}] *[\mathrm{R}]}
$$

in which:

$K_{\alpha}: \quad$ the affinity constant of the receptor $R$ to bind the ligand $L$;

[LR]: the concentration of the formed ligand-receptor complex at equilibrium; this fraction normally is equal to the bound fraction $(B)$;

$[L]$ : $\quad$ the concentration of the free, unreacted ligand at equilibrium $(F)$;

$[\mathrm{R}]$ : $\quad$ the concentration of the unreacted receptor at equilibrium. 
If the fraction $F / B$ is chosen as response metameter Ri/b and $Q$ is equal to the total receptor concentration, it can be derived that:

$$
\mathrm{R}_{f / b}=\frac{1}{\mathrm{~K}_{\alpha}[\mathrm{R}]}=\frac{1}{\mathrm{~K}_{\alpha}(\mathrm{Q}-\mathrm{B})}
$$

From equation [3], it is obvious that there is an inverse linear correlation between sensitivity and affinity constant. The higher the affinity constant the lower detection limits can be reached. The affinity constant is the most contributing factor to the sensitivity of an assay.

Jackson et al. (16) have derived an expression for the sensitivity as function of the relative error in the response metarneter and the affinity constant:

$$
\mathrm{s}=\frac{\frac{\delta \mathrm{R}_{f / b}}{\mathrm{R}_{\mathrm{f} / \mathrm{b}}}}{\mathrm{R}_{\alpha}}
$$

in which:

S:

Fils

$\delta R_{i, 0}$

$K_{\alpha}:$

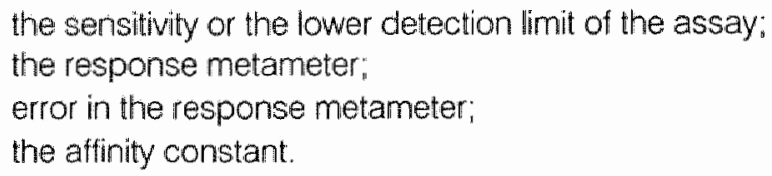

The error of the response metameter Rith is made up of two components:

- the error in signal measurement;

- the experimental error due to other origins.

The error in signal measurement can be calculated since radioactivity counting follows a Poisson distribution, in which the standard deviation of error equals the square root of the number of measured counts. The relative error is proportional to the standard deviation divided by the number of counts:

$$
\text { esignal }=\frac{\sqrt{\text { counts }}}{\text { counts }}
$$

At increasing counts the error in signal measurement reduces: $e=10 \%$ at 100 counts, 1 $\%$ at 10000 counts and $0.1 \%$ at 1000000 counts. Thus, an easy way to improve sensitivity of a PIA is to increase counting time. From a practical point of view, however, counting time must be limited to a reasonable value in order to have the assay finished. From a theoretical point of view, improvement of counting efficiency can reduce the sensitivity also, but in practice the obtained differences hardly will be statistically significant. Another possibility to reduce the sensitivity is to increase specific activity of the radioligand. This quantity is the ratio of the number of labelled ligand molecules within the tracer to the number of cold ligand molecules. An increase of specific activity results in more counts accumulated in the same counting time. The error of counting is reduced. 
Since the impulse rate in RIA is maximal at zero binding, an increase in count rate by an increased specific activity will further reduce the standard deviation and according to its definition sensitivity will also be improved.

The experimental error is derived from the manipulations within the assay and especially from pipetting.

If we assume the counting error to be negligible and the experimental error to be of the order of $1 \%$ than the maximal sensitivity achievable is given by the inverse of the affinity constant.

For a given affinity constant of $10^{12}[/ / \mathrm{mol}]$ the maximal sensitivity will be in the order of $10^{-14}[\mathrm{~mol} / \mathrm{l}]$.

The relationship between affinity constant and maximal sensitivity is presented by the uninterrupted line in figure 2.3a.

'COMPETITIVE' IMMUNOASSAY

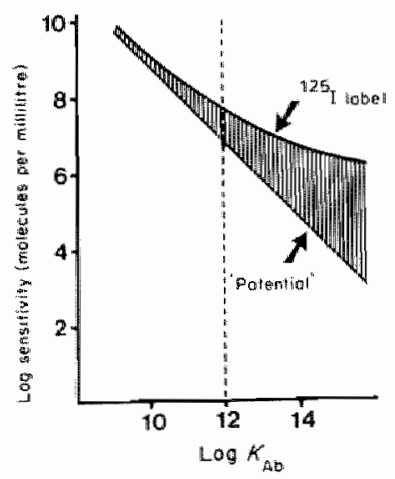

'NON-COMPETITIVE' IMMUNOASSAY

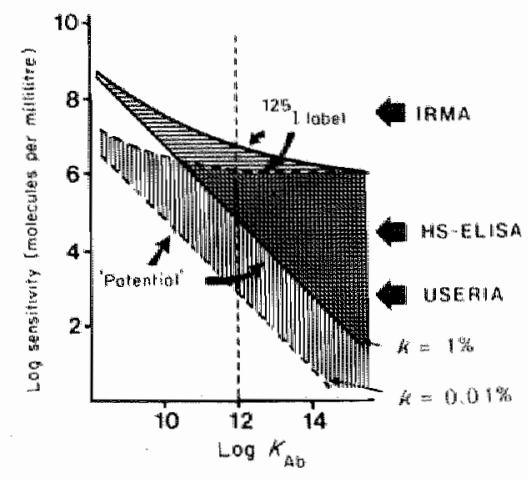

Figure 2.3a and 2.3b:

Assay sensitivity as function of equilibrium constant for various types of assays.

'Potential' sensitivities of competitive and non-competitive immunoassay systems as a function of the antibody equilibrium constant $(\mathrm{K})$. The value for e assumed for competitive systems $=0.01(1 \%)$; values lor $\mathrm{k}$ of 0.01 (1\%) (upper curve) and $0.0001(0.01 \%)$ (lower curve) have been assumed for non-competitive systems; on/nsb $=0.01$ (1\%) in both cases.

The ${ }^{125}$ label curves represent the predicted sensilivities attainable assuming labelling of analyte or antibody with ${ }^{125}$ (one atom per molecule) and "reasonable" values for counting efliciency and counting time, etc. Note that for aftinity constants below $10^{12}$ Umol (the upper limit, in practice, of antibody affinity), the sensitivity loss arising from the use of ${ }^{125}$ is small in the case of competitive sysltems, bul may approximale three orders of magnitude in the case of non competitive techniques. Note also that a non- competitive IRMA may display a sensitivity one to two orders of magnitude greater than a competitive $\mathbb{R} \mid A$ when based on antibody of identical aftinity. Arrows incicate typical assay sensitivities reported lor nom-compeitive IPMA. and for analogous enzyme-labelled antibody assays relying on lluorogenic [HS. ELISA (Shalev ell al., 1980)] and radioactive [USERIA (HIarris ef al.,1979)] subsirates. (With permission, Prof.Dr.R.Ekins)

In practice however, the counting error is not negligible and is of the same order of magnitude as the experimental error. As demonstrated above, sensitivity is a linear function of specific activity also.

Depending on the value of the specific activity one of the dashed lines in figure 2.3a will be applicable. 


\subsubsection{Non Competitive Immunoassays.}

The general equations for the reactions in a non competitive immunoassay using labelled antibody are:

$$
\begin{gathered}
s-R_{1}+L \rightleftarrows s-R_{1}-L \\
s-R_{1}-L+R_{2} \rightleftarrows s-R_{1}-L_{-}-R_{2}^{*}
\end{gathered}
$$

Thermodynamic laws can be applied just as for competitive immunoassays. Assuming infinite specitic activity of the labelled antibody ${ }^{*} R_{2}$, Ekins (17) has derived that the maximal sensitivity that can be achieved is equal to:

$$
\mathrm{s}=\frac{\mathrm{k} * \delta \mathrm{n}}{\mathrm{K}_{\alpha} \mathrm{nsb}}
$$

in which:

k : the fractional non specific binding of antibody;

Ka $\quad$ : the affinity constant of the solid phase coated antibody;

$\frac{\delta_{n}}{n s b} \quad$ the relative error in the estimate of the response due to non specific binding of the antibody.

As in competitive immunoassays, the maximal sensitivity achievable in non-competitive immunoassays is inversely proportional to the affinity constant of the antibody. Only the proportionality constants are different; i.e. $\delta \mathrm{R} / \mathrm{R}$ for competitive and $k \delta_{\mathrm{V}} / \mathrm{n}$.s.b. for non-competitive assays. From this difference, the gain in sensitivity of non-competitive assays in comparison to competitive assays can be explained: a reasonable assumption for the coefficient of variation in the non specific binding of the antibody is $1 \%$, while the assumption that the fractional non-specific binding of the antibody does not exceed $1 \%$ is reasonable too.

Thus:

$$
\begin{aligned}
& \frac{\delta_{\mathrm{n}}}{\mathrm{nsb}}=0.01 \\
& k<=0.01
\end{aligned}
$$

Substitution in equation [8] gives:

$$
\mathrm{s}=\frac{10^{-4}}{K_{a}}[\mathrm{~mol} / \mathrm{l}]
$$


In conclusion the maximal sensitivity achievable in non-competitive imnunoassays is about $10^{4}$ times better than in competitive assays. Like in competitue assays the specific activity plays a dominant role too at high affinity.

The practical attainable sensitivity deviates from the maxinal sensitivity in proportion to the decrease in specific activity. This phenomenon is graphicaly presented in figure $23 b$. It may be noticed that a reduction in sensitivity by a factor of one thousand can be achieved in changing the competitive assay with an affinity constant of $10^{12}[1 / \mathrm{mol}]$ to a non competitive assay with the same affinity constant.

However, in practice often polyclonal antibodies are used in RIA and RIA-like assays. Such antibodies can be produced having high values for the affinity constant (up to $10^{12}[1 /$ mol] $]$. In IRMA or IRMA-like assays most trequently monoclonal antibodies are used, but in general these have lower affinity constants (in the order of $10^{10}[1 / \mathrm{mol}]$ ). For this reason the gain in sensitivity is nol as great as could be expected theoretically. Our experience indicates that reductions in the lower detection limit by a factor 10 are easily achievable.

\section{The kind of tracer.}

In immunoassay one of the reactants must be labelled in order to detect the immunological reaction.

Since these assays generally are used to detect minor concentrations of ligand (in the order of magnitude of $\left.10^{-9}[\mathrm{~mol} / \mathrm{l}]\right)$ the detection of the label must be very sensitive. This sensitivity is comparable witth the recognition of only 1 person out of a group of one thousand million people. The problem can be resolved by asking that person to bear a flag great enough to be seen at long distance. Providing that nobody else bears a flag. the flag represents the label in immunoassay.

Radioisotopes have the potency of the sensitivity needed since natural occurring background is very low and activily can be measured in a reproducible way by means of beta or gamma counters.

In early days of immunoassays carbon-14 and tritium were used as labels. These are isotopes of common elements of organic material. The introduction of the label within the molecule to be labelled has the enormous advantage of an unchanged molecular structure and the labelled molecule behaves identical to the unlabelled molecule. However, both isolopes are pure beta emitters and the techniques needed to measure beta emission using liquid scintillation are labourious, expensive and they carry many pitfalls. In contrast, the measurement of gamma radiation is much more convenient and less expensive. Onlly few biological compounds contain elements of which radioisotopes are known that emit gamma radiation suitable for in-vitro detection: some examples are cyanociobalamin (vitamin B12) labelled with ${ }^{57} \mathrm{Co}$ and the thyroid hormones in which ${ }^{127} \|$ is exchanged for ${ }^{125}$ or ${ }^{131}$.

lodine isotopes may also be used to label molecules that otherwise could not be labelled because of the lack of suitable radioisotopes for its major elements. In fact, in proteins commonly present ring structures like tyrosine and histamine may incorporate one or two atoms of iodine just like the natural incorporation of iodine in thyroid hormones.

From a practical point of view ${ }^{125}$ lodine is very suitable, since its emission with an energy 
of $28 \mathrm{keV}$ is easy detectable with efficiencies of up to $75 \%$. Furthermore, it has a half-fife of 602 days long enough to pernit all manipulations like production of isotope, labelling. purification of radioligand, quality control and delivery to end-users to be performed within a reasonable period.

Therefore it is not surprising that from the beginning of the era of radiommunoassays ${ }^{125}$ lodine has been used extensively.

However, the major advantages of this isotope have become its major inconvenients too: firstly the detection of radicactivity necessitates special equipment and moreover special licenses. Nowadays, radioactivity has become unpopular and although the quantity of radioactivity used during the whole procedure of an assay hardly can be given any importance, further restrictions to its use will be imposed.

The long half-ife of ${ }^{125}$ lodine is the second inconvenient. In fact, due to this slow decay rate, the tracer is used in a very insufficient way. While the tracer will show activity for at least 600 days (10 times halt-life), the period of measurement is limited from minutes to hours! Furthermore, in the pre-measurement period the decay of tracer can induce serious radiodamage, changing the molecular structure of the radioligand. Such changes greatly influence the immunogenic character. These transformations will result in an assay sensitivity, which is worse than predicted from a theoretical basis.

From figure 2.3 it can be depicted that, it is necessary to search for labels of higher specific activity than commonly used radioisotopes. This is especially true for the non competitive immunoassays using labelled antibodies.

\section{Alternative Labels}

Other labels thave been introduced in immunoassay techniques. Since ${ }^{125}$ lodine is still the most widely used label, they are generally denominated 'aiternative labels'. Within the alternative labels three different classes can be recognized:

a: the enzyme label: the labelled enzyme activates (generally in a follow reaction) the transition of a substrate to a reaction product that is measured by photometric analyses.

b; the chemiluminescent label that contains a luminophore. Oxidation of this luminophore generates photons that can be detected by photometry.

c: the fluorescent label, characterized by a strong emission of photons if excited by photons of adequate energy.

\section{Specific activity of different labels.}

125 Iodine has a half-life of 60.2 days. After 14 half-lives, activity has reduced to less then $0.01 \%$ of the initial value. We assume all the atoms to have decayed in this period of $1.21 * 10^{6}$ [sec]. It is obvious that in order to have on average each second 1 atom ${ }^{125} \|$ decayed, it is necessary to have at least $1.21 * 10^{6}$ labelled molecules, which means that a high degree of labelling is necessary.

For the enzyme label the situation is much better; by using enzymes signal amplification can be pertormed: each labelled molecule can catalyze the reaction of substrate to 
reaction product more than once.

In theory great amplification factors can be attained. There are some practical restrictions resulting from the dynamic limits of the photometer and from substrate depletion. Chemiluminescent labels in theory yield 1 detectable photon per labelled molecule. In practice the quantum efficiencies are lower than $100 \%$ due to chemical and photometric quencing, but in general the specific activity is still much higher than that of radioactive isotopes.

Many detectable photons per labelled molecule may result from the excitation of fluorescent labels. This group of labels has a high specific activity. There are some practical problems too, since quencing, non specific fluorescence and phosplhorescence diminish the usability. Modern instrumental procedures with delayed measurements and the use of labels with a large Stokes shifl (as for example Europium) further enhance fluorescent techniques.

The specific activities of the various labels are compiled in table 2.1. It is obvious that chemiluminescent and fluorescent labels are most promising. In essential both are able to yield the same sensitivity, but the better signal-to-noise ratio of some fluorescent labels might favour their use rather than chemiluminescent labels.

Table 2.1: Specitic Aclivities of ${ }^{125}$ lodine and some Alternative Labels.

\begin{tabular}{|c|c|}
\hline Labell & specific activity \\
\hline${ }^{125}$ lodine & 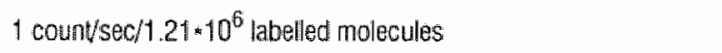 \\
\hline enzyme & depends on enzyme amplifications and photometric instrumentation \\
\hline chemiluminescent & 1 phaton/labelled nolecule \\
\hline fluorescent & many photons/labeiled molecule \\
\hline
\end{tabular}

\subsection{Analytical Precision and Accuracy}

Quality control is one of the most important tasks of the clinical chemist. Methods using immunochemical techniques particularly carry many pitfalls due to for example:

- the sigmoidal calibration curves generally found;

- the very critical amounts of antibody (antibodies) and (labelled) antigen (even in non competitive assays):

- temperature dependence of the equilibrium reactions;

- matrix effects;

- variability of non specific binding:

- high dose Hook effects;

- counting statistics;

- energy shifts of the counter; and so on. 
TSH [mU/1]

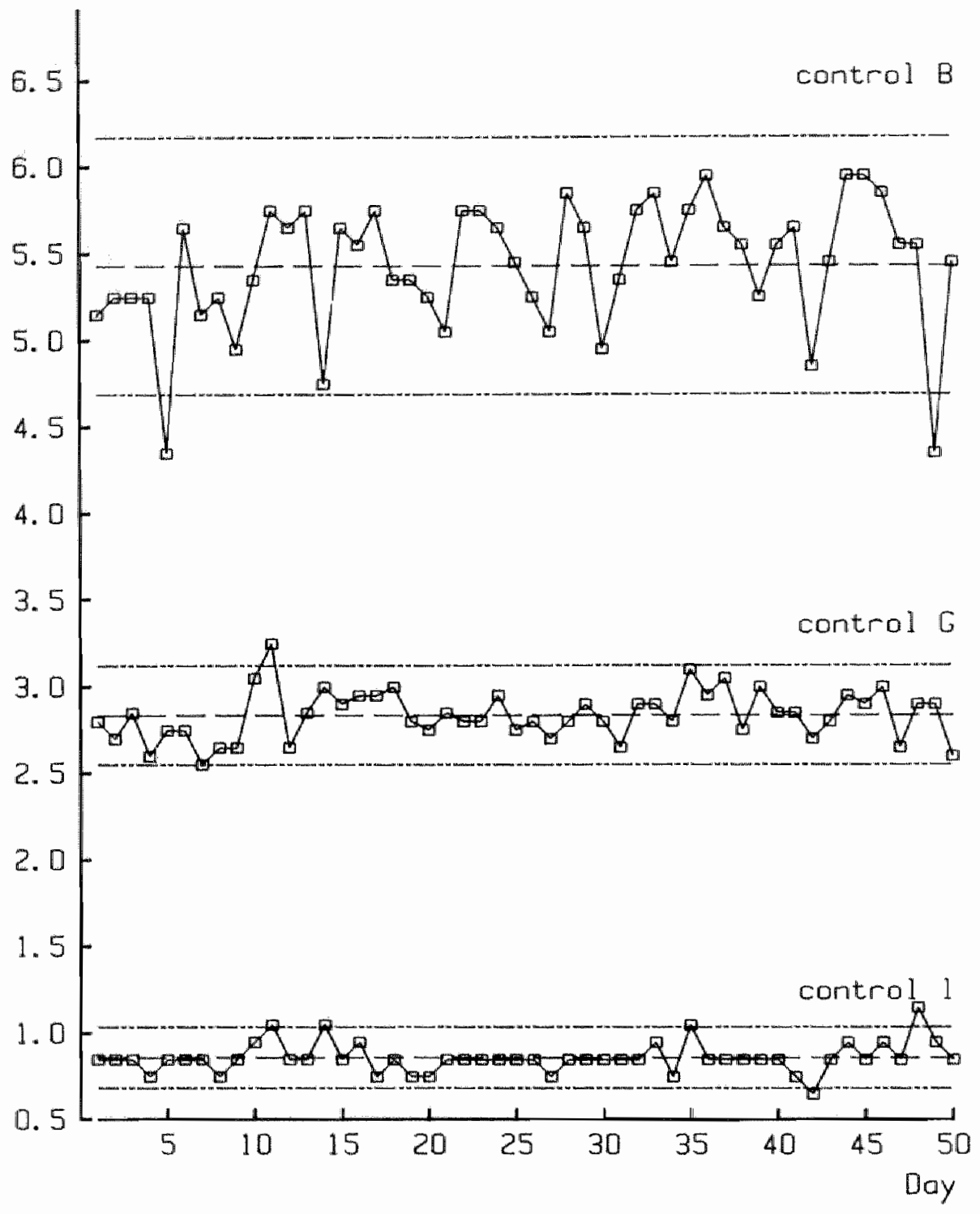

Figure 2. 4: Shewhart plot of TSH-restuits in control samples. 
To control all these variables in most laboratories quality control programs have been incorporated in daily routine. Although most assays are perfomed by using commercially available materials, the practitioners of immunoassay techniques are not discharged to verify that the results are reproducible and that they are clinical reliable.

Special attention must be paid to precision and accuracy since precision is important in the long time follow up of patients, while the accuracy is essential to obtain results comparable with those in other laboratories.

\subsubsection{Precision}

Experimental errors are governed by numerous factors among which the quality of pipetting, some characteristic properties of the sample (viscosity, lipaenic or hemolyzed) and instrumental errors like counting errors or quenching.

The analyst that performs the assay, induces random errors and perhaps systematic errors.

Occurrence of systematic errors leads to bias of the final results. They are difficult to perceive, since often true values are not known and definite methods for their determination are not available. This kind of error will be discussed later.

The overall random error, composed by the experimental error and the instrumental error: must be minimized. Before any optimization can be performed, however, the errors must be visualized. For that purpose several, mostly three, control samples are analyzed within each assay. After a certain number of assays standard deviation and mean values are calculated and a Shewhart graph is plotted. In this plot more parameters defining the assay can be incorporated such as sensitivity, $\mathrm{Bo} / T$, batch-numbers, etc. Such graphical presentations offer the possibility to observe long term stability at different concentration levels (interassay variation). If the same control samples are utilized in more laboratories, exchange of data permits to check the intra laboratory variance versus the inter laboratory variance. Typical Shevihart plots of TSH and LH are plotted in figures 2.4 and 2.5.

Shewhart plots do not offer a possibility to verify the intra-assay variation. This facility could be added but necessitales for at least one control sample to be analyzed more than once within an assay. This would lead to an inefficient ralio of the number of patient samples to the number of standards and controls.

A more convenient way is to calculate a precision profle based on the duplicates of standards, control samples and patient samples. The theory behind this proceclure has been developed by Ekins (18) and Hunter (19) and will not be discussed in detail. Precision profiles plotting concentration versus coefficient of variation permit the intraassay monitoring of the assay performance. They can be used for the evaluation of different assays or different assay procedures in order to optimize the critical steps. In figures 2.6 and 2.7 typical precision profiles are presented of a TSH and a LH assay Especialy in figure 2.6, the improvement of the IRMA methodology above that of RIA is clearly apparent.

\subsubsection{Accuracy}

The aim of each analysis is to detect the concentration of a well defined analyte. 


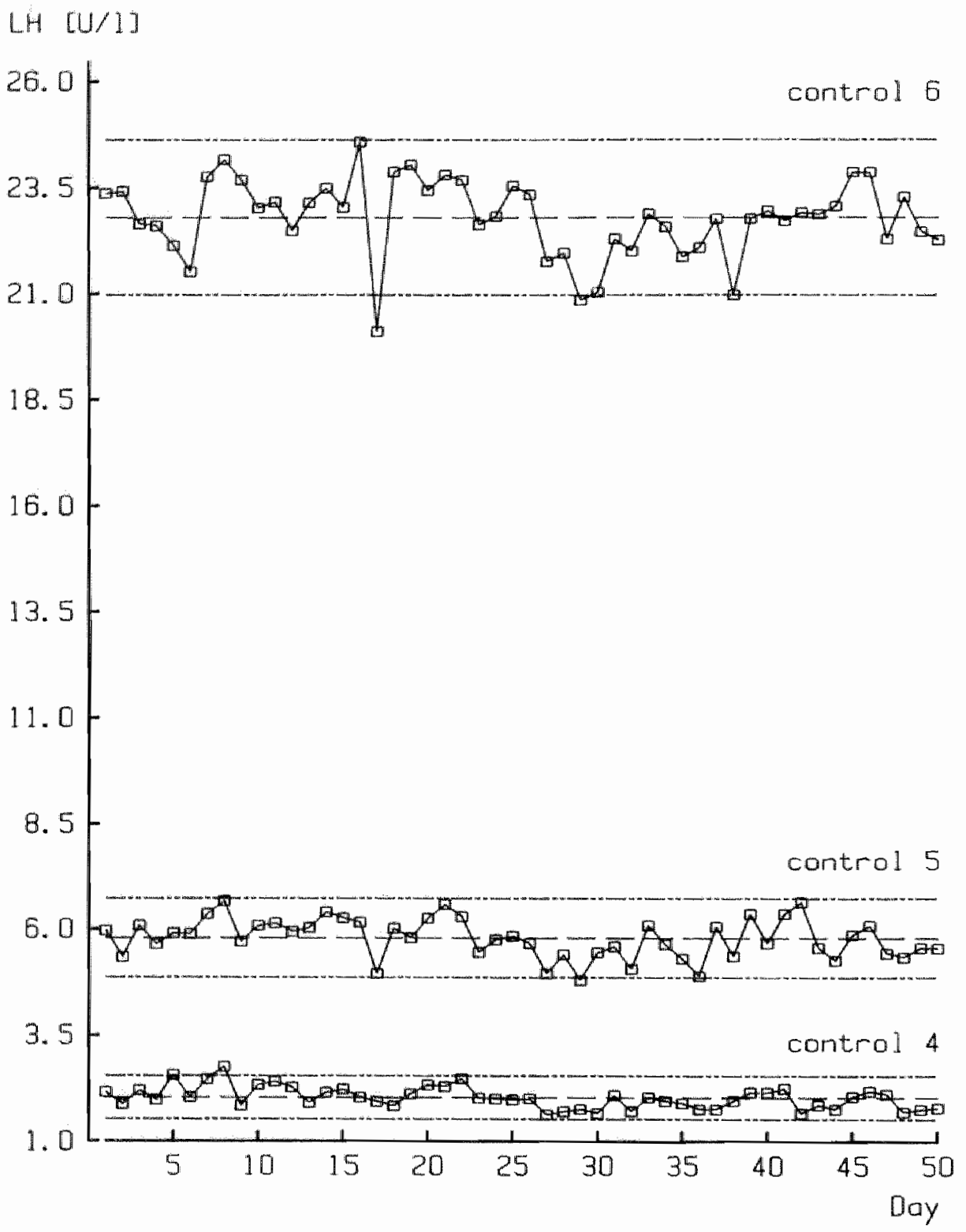

Figure 2.5: Shewhart plot of LH. results in control samples. 
The measuring process is the relative comparison of the response of an analyte in the system to the response of a series of dilutions of a standard solulion of known concentration. These ditutions are chosen so that the complete range of clinically relevant concentrations is covered. It is obvious that the production of the primary standard represents a crucial phase within the calibration processes, but unfortunately productions of such materials are very complicated. Extraction of biological material is necessary for the hormones of interest in this study. TSH and LH, as there are no (not yet) DNA recombinant antibady production methods available.

Since the starting material of each manufacturer is different, products will be obtained that may show more or less important differences: subunits may be present, generally wihout having biological activity; fragments can be present that have biological activity while the immunoactivity of possibly present iso-hormones may be different from biological actiwity. Furthermore desiallyzation and/or proteolytic degradation can contribute to heterogeneily of the extraction product.

Next the quality of the standard is strongy dependent on the character of the starting material: extraction from urine samples can give a totally different reference preparation than the extraction of serum or pituitary tissue.

Standardization is absolutely necessary, especially if results have to be compared with those of other laboratories.

International reference preparations supported by the World Health Organization are available that have been attributed an assigned value for a particular homone. However. since other hormones may be present in these reference preparations, it is important to verify that these do not interfere with one of the antibodies of the assay.

The reference preparations are distributed in limited quantities by the Medical Research Council (M.R.C.) in London (U.K.) and the National Institute of Health in Bethesda (U.S.A.). In each of the preparations the amount of hormone of interest has been determined by wo totally different procedures, one being the immunological way and the other being the biological way. Various laboratories all over the world have contributed to the establishment of a reference value for that particular hormone. The preparations are intended to be used as long term primary standards and it is desirable that all melhocs are calibrated against these materials. For this reason the concentrations are presented in International Units rather than in mass units. Before new preparations are distributed they are calibrated against the old ones, taken into account new dewelopments in terms of specificity, biological activity and purity of the hormone.

Both the W.H.O. reference preparations for the homones of interest in this study, i.e. TSH and LH have been or are going to be replaced by newer preparations. Hence, the reference preparation for TSH 68/38 (M.R.C.68/38) has been replaced by preparation $80 / 558$.

In our study, we used the Boots-Celltech assay for the determination of TSH. We did not observe any difference between the response of the assay for both standards. Howewer, it must be realized that with other assays or with different methodologies both W.H.O. preparations can have completely different responses. This depends on cross reactivity and different analytical sensitivities of the used assay systems. The reference preparation for LH (M.P.C. 69/104) is now almost exhausted. A new preparation (M.R.C.78/556) has already been subject of some study. (20)

in our laboratory the calibration of both the assays for TSH and LH has been verified 
c..

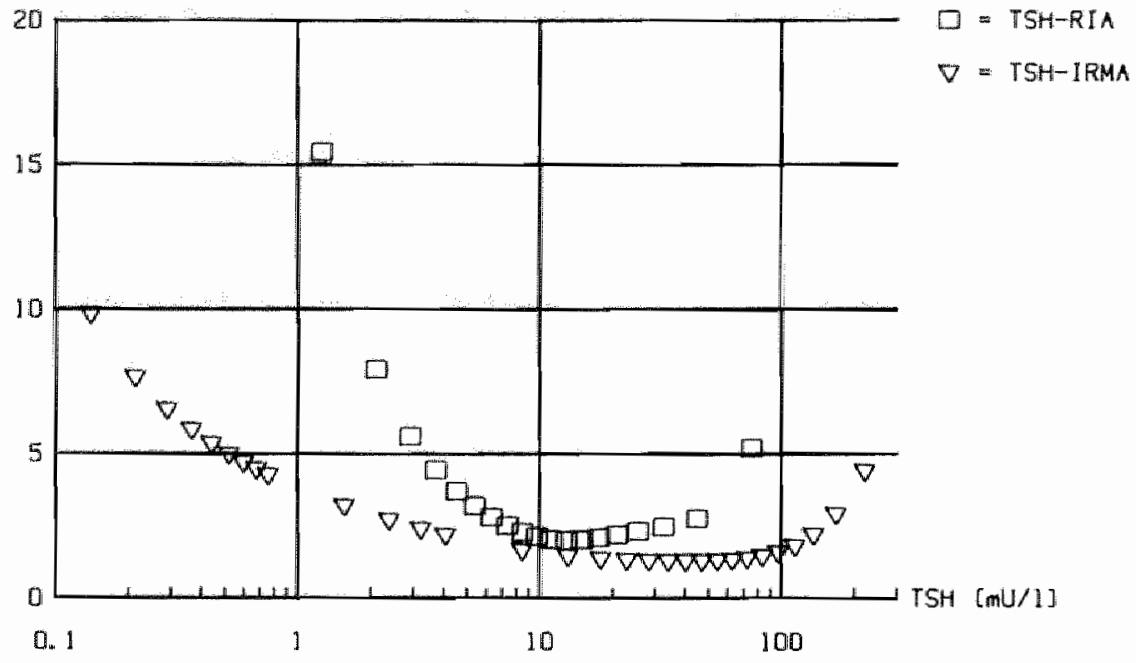

Figure 2.6: Precision prolile of TSH-PIA and TSH-IRMA.

C. V.

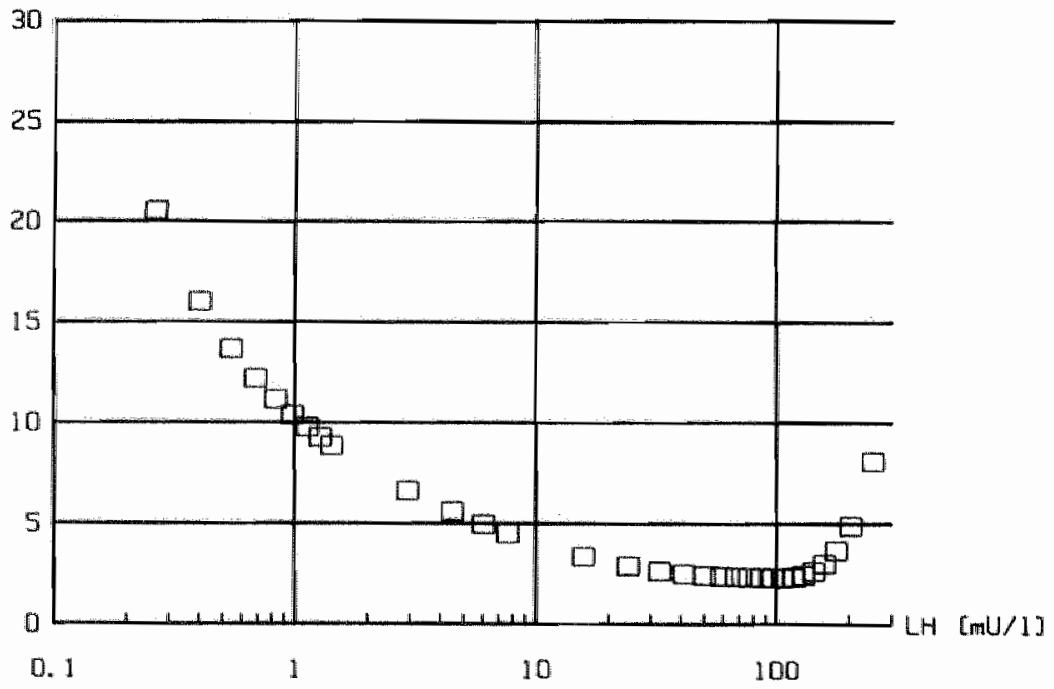

Figure 2.7: Typical precision prolile of LH.IPMA. 
against the W.H.O. materials.

This will be discussed in a next section. In daily routine four control samples are analyzed in each assay and by means of Shewhart graphs, it is verified that possible blas within the calibration remains within statistical acceptable limits.

\subsection{Sensitive Estimation of Thyroid Stimulating Hormone (TSH)}

Alreacy in 1974 the description of a research method for TSH was published (21) in which a sensitivity of $0.005 \mathrm{mU} / \mathrm{l}$ was reported. However, the execution of the whole assay demanded about 5 days while otherwise the assay could be classified as very cumbersome. This assay could not be used for routine purposes.

As has been exposed in chapter 1.5, the sensitivity and the specificity as offered by all commercially available PIA methods for the determination of TSH was insufficient to discriminate between patients with hyperthyroidism and euthyroid patients. In 1984 the prodiuction and especially the selection procedures of monodonal antibodies were improved in such a way that antibodies with high specificity and acceptable affinity constants were available. The first assay that could be purchased in the Netherlands was the Sucrosep ${ }^{\text {rw }}$-TSH assay of Boots-Celltech, Slough, (U.K.). Many publications followed in short time in which the clear improvements of this assay compared to others were elaborated $(22,23,24)$. Especially the possibility to discriminate samples of euthyroid patients from samples of hyperthyroidic palients and the possibility to use this assay as a first screening test for dysthyroidism $(25,26)$ have been discussed in literature Furthermore the assay has been proposed by authorities within thyroidology to be a replacement for the T.R.H.-stimulation test $(26,27)$.

Since this assay has been used for all analyses that have been performed within the scope of this thesis, the practical procedure is described.

\subsubsection{Practical Performance of the Boots-Celltech Sucrosep ${ }^{\text {TM}}-$ TSH assay.}

The Boots-Celltech Sucrosep ${ }^{1+}$-TSH assay combines two principles that contribute clearly to the improved sensitivity and specificity.

Firstly, the assay is a two site IPMA in which an excess quantity of anibody labelled with ${ }^{125}$ lodine is allowed to react with TSH molecules of the sample to form a labelled antigen-antibody complex (figure 2.8 upper part).

A second antibody coupled to a solid phase, is added to the reaction mixture. This antibody recognizes another epitope on the antigen and subsequently a complex is formed that looks like a sandwich enclosing the antigen (figure 2.8 lower part).

TSH consists of two distinct subunits, the alpha- and the beta-subunit. It is assumed that one of the antibodies reacts with the beta-subunit and the second one to an epitope on the complete molecule that contains the coupling site of the beta- with the alpha- subunit. A reaction with single alpha- or beta-subunits is excluded. However, it can not be excluded, as has been depicted in chapter 2.3, that some of the molecular forms of TSH will not react in the sandwich despite the fact that they have biological activity.

Furthermore, it is possible that some biological inactive forms of the TSH molecule show binding properties to the antibadies. However, our clinical experience has not shown 


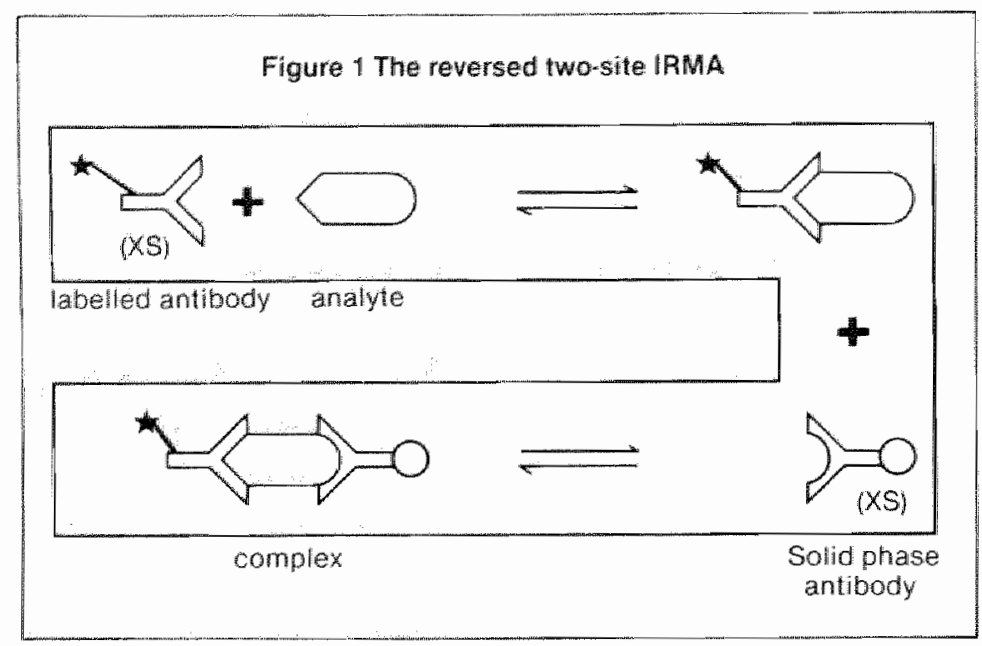

Figure 2.8: Principle of two sille IFMA.

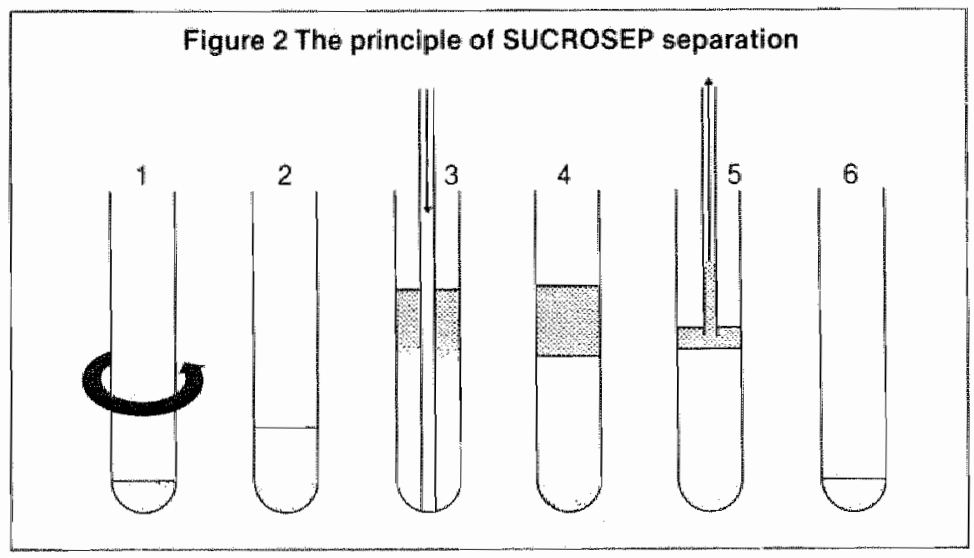

Figure 2.9: The principle of Sucrosep separation.

such false positive estimates. For other commercial preparations such errors have been reported (28).

The second reason for the high sensitivity and specificity of the Sucrosep " the used separation step. It is based on solid-phase technology using Pharmacia's Sephacryl S300. This separation method is described by Wright and Hunter in 1983 (29). The solid phase S300 falls relatively quickly under gravity without further instrumental acceleration. During the incubation of the reaction mixture this spontaneously accurring process is avoided by continuous agitation of the reaction vessel (figure 2.9-1).

Thereafter washbuffer is added and the solid phase is allowed to settle (figure 2.9-2). This step is required to enhance the separation efficiency.

Next a relative dense sucrose solution is added at the bottom of the reaction vessel by 
means of a special device (figure 2.9-3). The incubation mixture is raised above the sucrose solution, the solid phase falling through it to the bottom of the vessel (figure 2.9-4). After 15 minutes the upper layer is removed by means of another dedicated device aspirating the upper layer and most of the sucrose solution (ligure 2.9-5). The bottommost layer contains the solid phase, a fraction of which consists of labelled sandwich-complex that can be counted (figure 2.9-6). Highest performance in sensitivity and precision are reached by performing the sucrose wash step twice.

\subsubsection{Characteristics of the Boots-Celltech Sucrosep ${ }^{\mathrm{TM}}{ }^{\mathrm{T}} \mathrm{TSH}$ assay.}

\section{Cross reactivity.}

The cross reactivity of the sucrosep TSH assay is reported to be excellent. The values of the percentage cross reactivity of TSH from other species and of some physiological important pituitary hormones are reported in table 2.2 .

Table 2.2:

cross reactivity in Sucrosep ${ }^{\text {* }}$-TSH assay.

$\begin{array}{ll}\text { hormone } & \text { Cross reactivity * } \\ \text { Bovine TSH } & \\ \text { murine TSH } & \text { none } \\ \text { Prolactin } & \text { none } \\ \text { LH } & 0.004 \\ \text { FSH } & 0.009 \\ \text { hCG } & 0.004 \\ \text { h. none }\end{array}$

* "defined as apparent mass TSH divided by mass cross-reactant.

It must be noticed that the observed cross reactivities possibly are due to contamination of the tested pituitary extract with TSH. This can be deducted from interference studies with $\mathrm{Gn}-\mathrm{RH}$. Stimulation of healthy volunteers with Gn-RH resulted in increased concentrations for $\mathrm{LH}$ and FSH, but the TSH concentration did not change significantly. For hCG a slight apparent decrease was described leading to a lower TSH response in the first trimester of pregnancy (table 2.3). Such interferences are important in the interpretation of (combined) RH stimulation tests.

Table 2,3:

Intterference in Sucrosep ${ }^{\text {Tw }}$-TSH assay.

$\begin{array}{ll}\text { Hormone } & \text { Interterence * } \\ \text { LH } & 1.00 \\ \text { FSH } & 0.98 \\ \text { nCG } & 0.87\end{array}$

*: detined as abserved TSH concentration divided by expected TSH concentration 


\section{Sensitivity}

The mean sensitivity reported by the manufacturer was 0.05 [mU/] with a range of 0.03-0.06 [mU/l]. We found a sensitivity that was in good agreement with these values, the mean sensitivity of 20 assays (defined as 2.5 times the standard deviation from the zero standard) being 0.034 [mu/].

\section{Precision.}

In various paragraphs throughout this thesis "precision profiles of TSH- assays are reported. It can be concluded that the precision is excellent and that within the dynamic range there is a broad region with coefficients of variation below $5 \%$. This feature offers the possibility to study clinical conditions with only minor changes in TSH concentration.

\section{Accuracy.}

The accuracy of the TSH assay was tested by dilution studies of the reference preparation MRC $80 / 558$. The observed line is plotted in figure $2.10 \mathrm{a}$ and the data are presented in table 2.4 .

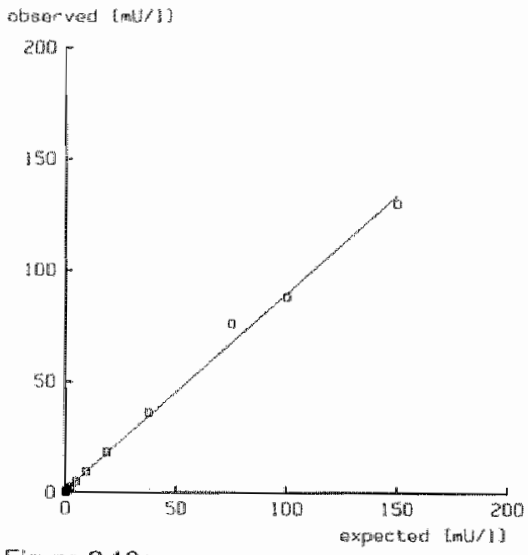

Figure 2.10a:

The recovery of MHC 80/55e in "TSH.assay.

[all samples; $Y=0.89 * X+1.01 ; r=1.00$;

$N=13$; classical regression $X$ on $Y)]$

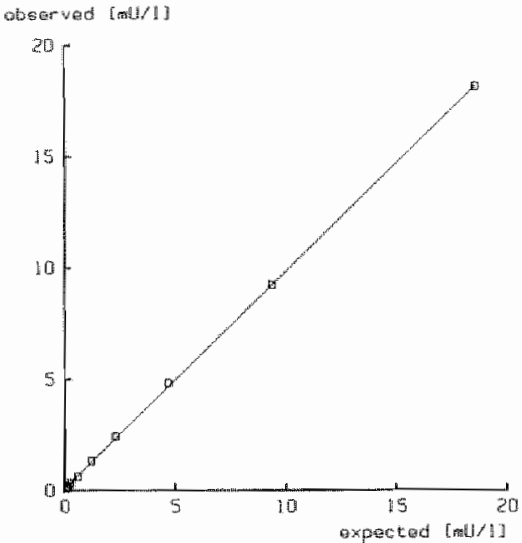

Figure 2.10b:

The recovery of MRC $80 / 558$ in TSH-assay. [subselection of samples with values below $20 \mathrm{mU} /$;: $Y=0.97^{*} X+0.09 ; r=4.00 ; N=9$

The coelticient of correlation $(r=0.9977)$ is excellent. The value for the regression coefficient (slope) of 0.8850 is not significantly different from 1, while the intercept was not significantly different from 0 . Of course the most concentrated solutions of the reference preparation have an important contribution to the coefficient of regression. For this reason, in figure $2.10 \mathrm{~b}$ a subset of the data is plotted with all values below $20[\mathrm{mU} / \mathrm{l}$. The coefficient of regression and the coefficient of correlation further improve in comparison with the original set of data.

Neither the slope nor the intercept are significantly different from their ideal values of 1 resp. 0. Earlier recovery siudies with MRC 68/38 showed parallel results. It can be concluded that the accuracy of the sucrosep ${ }^{\prime \prime}$-TSH assay with respect to the confidence limits of regression, is correct. 
Table 2.4:

Recovery of M.A.C. reference preparation $80 / 558$.

$\begin{array}{lcr}\begin{array}{l}\text { Theoretical TSH } \\ \text { concentration in } \\ \text { [mU/] }\end{array} & \begin{array}{l}\text { Observed TSH } \\ \text { concentration in } \\ \text { [mU//] }\end{array} & \begin{array}{l}\text { Recover } \\ \text { in } \%\end{array} \\ 150 & 130 & \\ 100 & 88 & 86.7 \\ 75 & 76 & 88.0 \\ 37.5 & 35.9 & 101.3 \\ 18.6 & 18.1 & 97.0 \\ 9.4 & 9.2 & 97.3 \\ 4.7 & 4.8 & 97.9 \\ 2.3 & 2.4 & 102.1 \\ 1.2 & 1.3 & 104.3 \\ 0.59 & 0.62 & 108.3 \\ 0.26 & 0.31 & 105.1 \\ 0.15 & 0.17 & 119.2 \\ 0.07 & 0.10 & 113.3 \\ & & 142.8\end{array}$

\subsubsection{Comparison of Sucrosep ${ }^{\mathrm{rm}}$-TSH with other assays.}

After the introduction of the Boots-Celitech Sucrosep ${ }^{\text {rm }}$-TSH assay, a lot of assays on commercial basis have been introduced.

Since our choice for the assay of Boots-Celltech was based primarily on the fact that it was the first assay commercially available, we have verified repeatedly that accuracy and precision were still as good as possible when compared to other assays. During the time of preparation of this thesis, the Sucrosep ${ }^{\text {rn }}$-TSH assay has been compared to five assays. All the assays were of the coated tube type, i.e. instead of coupling of the antibody to particles, the first antibody is covalently coupled to the tube. The main inconwenience of this type of assay is the impossibility to verify for non specific binding. Furthermore, it is difficult to verify homogeneity of the coating.

For commercial reasons, the name of the manutacturers but one are not mentioned, since the purpose of the comparison was to justify continued use of the Sucrosep ${ }^{1 m}$. TSH assay. One of the coated tube assays is produced too by Boots-Celltech, Slough, U.K., but in this assay antibodies different from those in the sucrosep assay were used. Probably, this has been done, because of the impossibility to couple the first antibody from the sucrosep assay covalently to the solid phase. Apparently, the use of a different first antibody necessitated also the use of another second antibody.

One of the coated assays was a chemiluminescent method in development, using a acridinium ester as label. The photons were delivered by injection of $\mathrm{H}_{2} \mathrm{O}_{2}$ in the with $\mathrm{NaOH}$ alkalinized solution. 


\section{Precision and dynamic range of TSH-assays}

In tigure 211 the precision profiles of six assays (5 coated tube assays and the sucrosep assay) are plotted.

C...

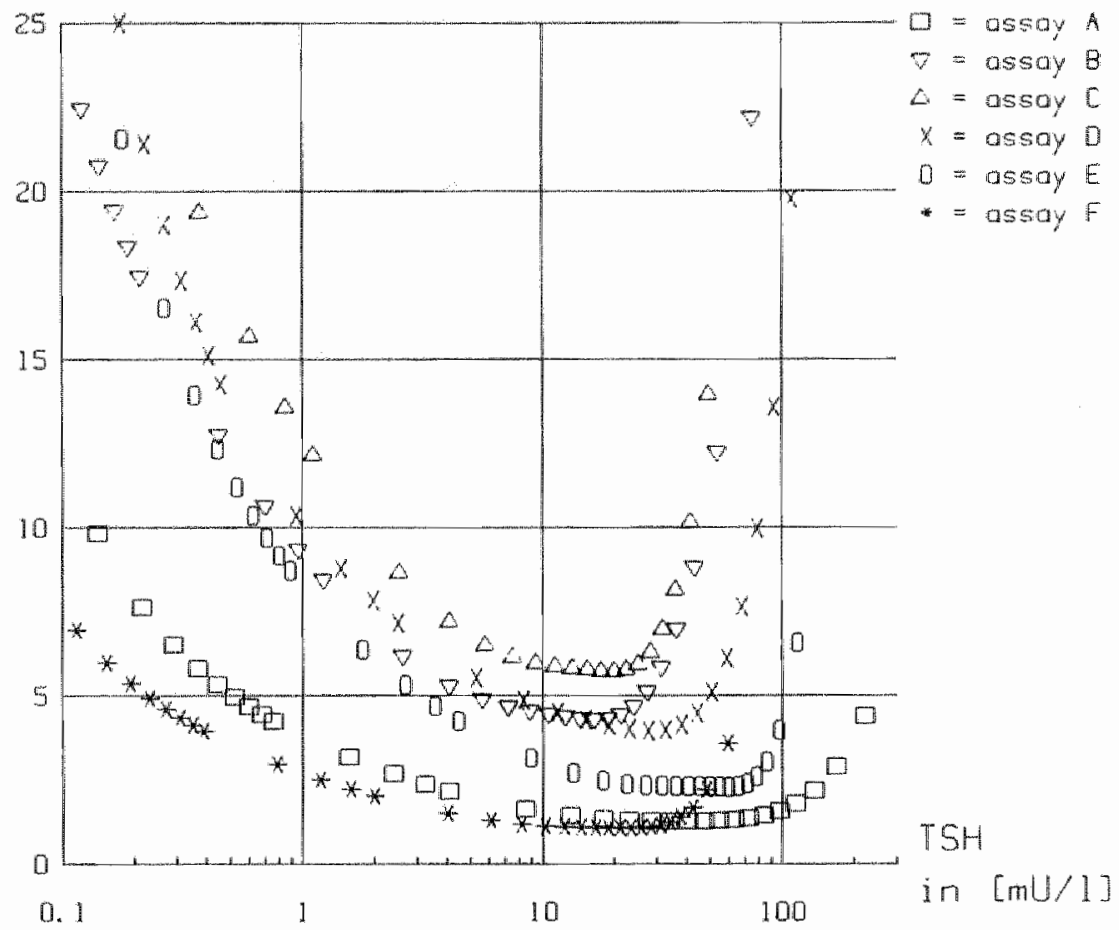

Figure 2.11: Precision proliles ol various TSH-assays.

From these plots the dynamic ranges have been derived characterized by coefficients of variation below $5 \%$ and $10 \%$. These have been compiled in table 2.5 . Since the dymamic range of an assay depends on the values of the standards included in the kit, the lowest (above zero) and highest standard concentrations are figured too. The values of the previous PIA-method are incorporated in the table too. It is obvious, that all IRMA-methods pertorm better than the RIA-method.

It must be realized that most calculations are based on one or two runs performed by skilled technicians. However, long term experience with one type of assay always influences the precision of an assay in a positive way.

From both figure 2.11 and table 2.5, it can be concluded, that the Boots Celltech Sucrosep" same manu-facturer is showing precision that is slightly better than that of the sucrosep ${ }^{n+1}$ - TSH assay. 
Sensitivities of different TSH assays.

The sensitivities calculated from the precision profiles at 2.5 times the standard deviation from the zero standard have been incorporated in table 2.5. It can be concluded that kits $A$ and $F$ have the best sensitivity. Little experience with $k i t F$ is, however, available.

TABLE 2.5: Precision and dynamic range of TSH assays.

$\begin{array}{lllllll}\text { Assay Type } & \begin{array}{l}\text { Standard values } \\ \text { lowest }\end{array} & \text { Sensitivity } & \begin{array}{l}\text { Dynamic range I Dynamic range il } \\ \text { C.V. }<5 \%\end{array} & \begin{array}{l}\text { C.V. }<10 \% \\ \text { hight }\end{array} \\ \text { A } & \text { Sucro } & 0.08 & 244 & 0.02 & 0.45-244 & 0.14-244 \\ \text { B. } & \text { c.t. } & 0.15 & 50 & 0.03 & 4.5-25 & 0.8-50 \\ \text { G } & \text { c.t. } & 0.15 & 50 & 0.06 & \text { not applicable } & 2.0-40 \\ \text { D } & \text { c.t. } & 0.10 & 50 & 0.08 & 6.0-50 & 1.2-80 \\ \text { E } & \text { chemie } & 0.10 & 100 & 0.09 & 3.0-100 & 0.6-? ? \\ \text { F } & \text { c.t. } & 0.15 & 50 & 0.01 & 0.2-50 & 0.05-50 \\ \text { G } & \text { RIA } & 1.0 & 60 & 0.94 & 3.5-75 & 1.5-60\end{array}$

(All values are given in [mu/l]; c.t.icoated lube)

\section{Comparison of patient samples.}

It was not possible to study the recovery of the M.R.C. reference preparations in all kits. In order to evaluate the accuracy of the assays, the kits have been compared to each other using patient samples.

In figures 2.12 to 2.16 the regressions of these data versus the results obtained with the sucrosep TSH are plotted. In all assays only the means of duplicate values have been taken into comparison. The regressions have been restricted to values between 0 to 15 [mU/l]. The regression characteristics have been compiled in table 2.6.

The assays $B, C$ and $D$ have significantly different coefficients of regression compared to assay $A$. In consequence, it may be assumed that something is wrong in the calibration of the assays. Assay $A$ has proven to be well calibrated versus MRC $80 / 558$ (figure 2.10) and versus MRC $68 / 38$. However, all manufacturers of assays $B, C, D$, and E calibrated their assays also versus one of two MAC-preparations. For this reason, no differences should be present between different kits. However, from personal information from the manufacturers, it may be concluded that they did not find a 1 to 1 similarity of both MRC-preparations. Undoubtedly, the differences in the epitope recognition of the used antibodies lead to different calibration of the assays. Within the same manufacturer (assays $A$ and $F$ ), although equipped with entirely different antibodies, no differences in calibration are observed. 
Table 2.6: Fegression characteristics of vanious TSH-assays.

(in comparison to the Sucrosep ${ }^{\text {gu }}$-TSH assay.)

assay N (TSH $<15$ (mU/l) Slope Intercep correlation coefficient

$\begin{array}{lllll}8 & 49 & 0.8622 * 0.0722 * * & 0.9864 \\ C & 15 & 0.7098 *-0.2342 & 0.9906 \\ D & 15 & 0.7728 *-0.0276 & 0.9966 \\ E & 45 & 0.8030-0.7851 & 0.8837 \\ F & 40 & 1.0233-0.0062 & 0.9893\end{array}$

* : statistically different from $1.0, p<0.01$, * : statistically different from $0.0, p<0.01$

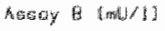

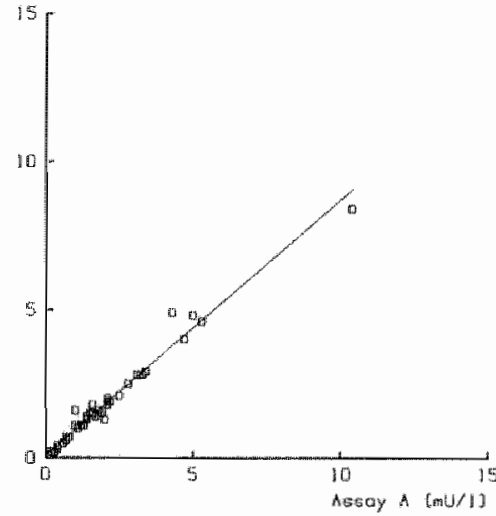

Figure 2.12: Regression of TSH-assays: assay $A$ versus assay $B$.

$\left[Y=0.86^{*} X+0.07 ; r=0.99 ; N=49\right.$; othogonal regression]

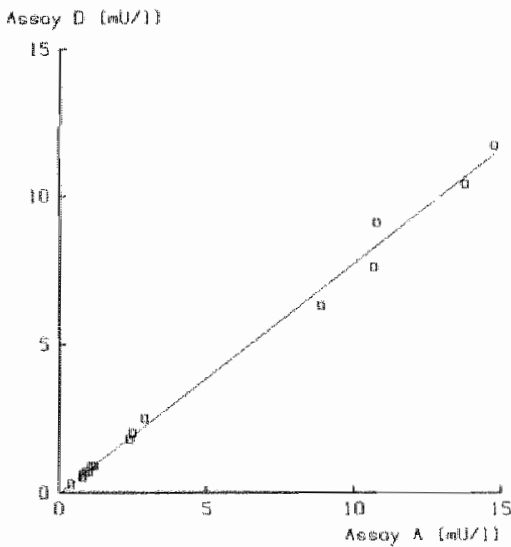

Figure 2.14: Fegression of TSH-assays: assay $A$ versus assay $D$.

$\mathbb{Y}=0.77 * X-0.03 ; r=1.00 ; N=15$;

othogonal regression]

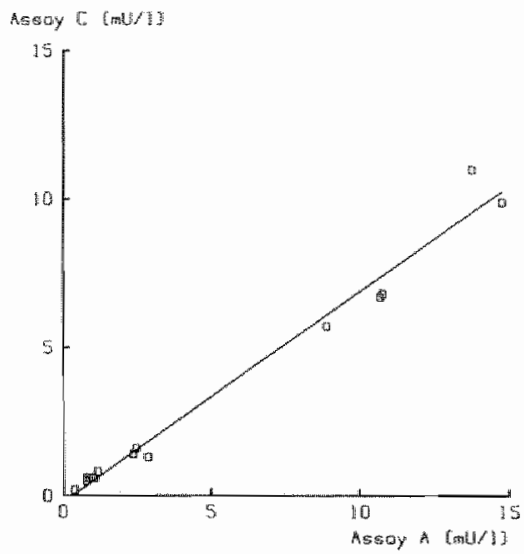

Figure 2.13; Fiegression of TSH-assays: assay $A$ versus assay $C$ $r Y=0.71 * X-0.23 ; r=0.99 ; N=15$ othogonal regression]

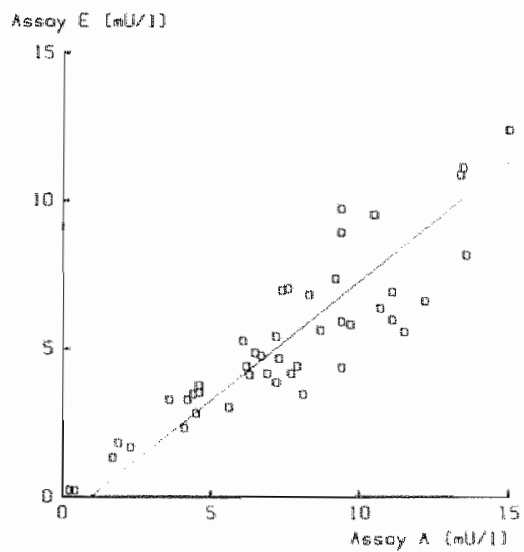

Figure 2.15: Regression of TSH-assays: assay $A$ versus assay $E$. $[Y=0.80 * X-0.79 ; r=0.88 ; N=45 ;$ othogonal regression] 


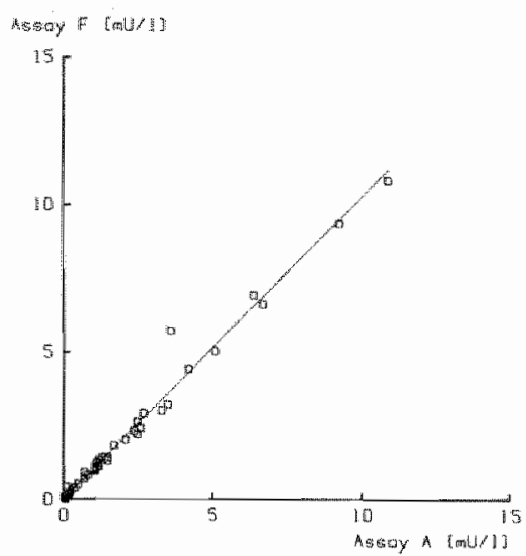

Figure 2.16: Regression of TSH-assays: assay $A$ versus assay $F$.

$[Y=1.02 * X-0.01 ; r=0.99 ; N=46$;

ofhogonal regression]

\section{Diagnostics errors due to misclassification.}

In practice it will be impossible to obtain identical data using different kits. As stated above, probably all kits have different combinations of antibodies. The recognized epitopes do not necessarily correspond to each other and so it is possible that TSH reactivity varies between reagents from different kits. Receiver-Operating-Curves (ROC-curves) can be helpful to objectify these differences, but this technique needs the analysis of a lot of samples, preferentially having values near the decision limits of the assays. Unfortunately, this was not possible for practical reasons.

Nevertheless, the samples that have been used for the regression analysis of table 2.6, have been compared to the reference interval of the TSH- assay and the number of occurrences has been noted that biochemical classification in hypo-, eu- and hyperthyroidic did not correspond in both the assays. The results are presented in table 2.7 .

Table 2.7: Misclassiticalion errors of TSH assays.

$\begin{array}{llll}\text { assay } & N \text { (samples) } & \text { N (errors) } & \text { Percentage error } \\ \text { B } & & & \\ \text { C } & 49 & 2 & 4 \\ \text { D } & 15 & 1 & 7 \\ \text { E } & 15 & 1 & 7 \\ \text { F } & 45 & 7 & 16\end{array}$

It must be realized, that only a limited number of samples were compared. The table has only been listed in order to demonstrate that it is very important to determine the reference values of the routine assay in each laboratory. Reference values from other laboralories, 
determined with different kits can not be used.

From the figures the conclusion is justified, that the Boots-Celtech Sucrosep "' "TSH assay

is one of the best with regard to accuracy, precision and long- ferm stability.

\subsection{Sensitive estimation of Luteinizing Hormone $(\mathrm{LH})$}

Mostly, RIA methods are used tor the assessment of the pituitary hormones with the exception of TSH. Prolactin was the second hormone being analyzed by IRMA methods, but the conversion of RIA to IRMA is relatively recent and still some IRMA methods do not use monoclonal antibodies.

Actually, the manufacturers are spending a lot of energy in the development and acceptance of monoclonal or oligoclonal LH and FSH assays. A major problem for acceptance of these methods in daily routine is the lack of clinical problems dealing with very low hormone concentrations. In literature the presence of at least five different iso-hormones for LH has been reported (19). The questions arising from this coexistence of iso-hormones are responsible for the little consideration given to increased specificity in the case of LH.

Nevertheless, for the understanding of problems within the fine regulation of the pituitary-gonadal-axes, it is necessary to use as sensitive and as specific assays as possible even if it would be necessary to use more, than one assay for LH. The use of oligoclonal assays (assays using a mixture of two or little more distincl monoclonal antibodies) theoretically, can be of profit, but a lot of clinical work must be done before such an assay can be validated for clinical use. The comparison of the results of an assay with bioassays is an important tool, but it must be realized that the chosen bioassay might be a bad reflection of the in-vivo system. For example, it can be imagined that some clinical states are due to changes in the pulsatile secretion of the hormones by the pituitary.

If the hormones have a rapid turnover rate in peripheral circulation, i.e. are immediately modified (sialyted or glycosylated, for example, when passing through the liver) the result is that, in circulation at least two hormones are present generally both showing bioactivity. The half-life of the hormome increases and consequently, it is clifficult lo detect minor chenges in secretion rate by the pituitary if not very sensitive assays specific for the modified hormone are used. Bioassays generally, can not offer this degree of specificity. Much is unknown about the biological functions of the various iso-hormones. It has been demonstrated that male $\mathrm{LH}$ has the same electrophoretic properties as $\mathrm{LH}$ in postmenopausal women and that $\mathrm{LH}$ of pre-menopausal women differs from $\mathrm{LH}$ of postmenopausal women (30).

However, up to now it is impossible to attribute to each distinct function of $\mathrm{LH}$ in man a particular molecular contormation of the $\mathrm{LH}$-molecule. Moreover, some functions of $\mathrm{LH}$ in man might be unknown untill now.

In consequence, the choice of an assay system is arbitrarily: there are only two conditions: firstly the assay must be very sensitive and secondly the assay must be specific for only one molecular structure of the LH. However, although electrophoretically distinguished forms of LH are known, for the moment it is not possible to chose an antibody based on a specific electrophoretic behaviour. Specificity is a matter of trial and 
error: once an antibody system chosen, it is necessary to verify exactly the clinical conditions in which LH molecules are produced that react with the selected couple of antibodies.

If monoclonal antibodies are used, at least specificity is as high as possible, but there is a risk that structures are recognized other than the structures looked for and possibly no relevant information will be found when comparing the results of the assay to clinical conditions.

Aware of the above mentioned problems and with the experience of the sucrosep ${ }^{\text {in }}-\mathrm{TSH}$ assay, we started with the Boots-Celltech sucrosep ${ }^{\text {TM }}$ LH-assay in the first part of the study.

In order to look for differences in responses caused by the recognition of different epitopes, we used in LH time series (chapter 5) another monoclonal based IRMA. distributed by Serono, Itally.

Comparisons have been made between both assays. In figure 2.17 the precision profiles of both assays have been plotted. A comparison has been made of the results of 33 patient samples. This regression is plotted in figure 2.18. The slope of the orthogonal regression line is significantly different from 1.0 ; the intercept is not significantly different from 0 .

This could mean that the standards of the assays have been calibrated differently. However, the recovery of the M.R.C.-preparation is correct. Probably, in the M.R.C. preparations fragments of $\mathrm{LH}$ molecules or $\mathrm{LH}$ molecules with different tertiary structure are present, which are measured in one of the assays and not in the other. It is impossible to verify the correct reason for this difference, since at the moment the new M.R.C.

preparation probably containing less cross reacting substances is not yet available. Since all samples of our study have been analyzed with the Serono assay, no complications with regard to the interpretation of results have to be expected.

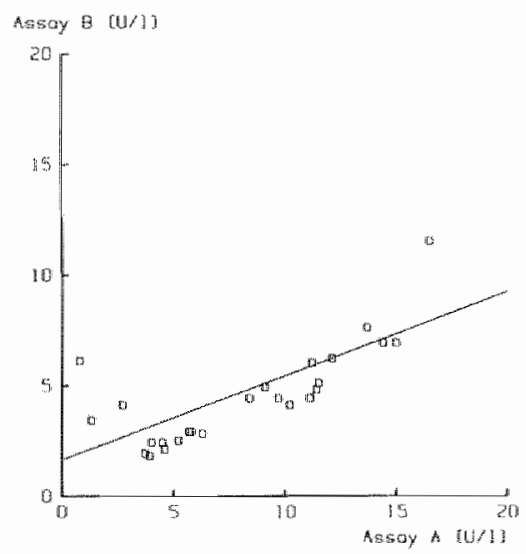

Figure 2.18: Pegression of Boots-LH (A) versus Serono-LH (B). $[Y=0.38 * X+1.63 ; r=0.98 ; N=33 ;$ orthogonal regression $]$ 
C. $V$.

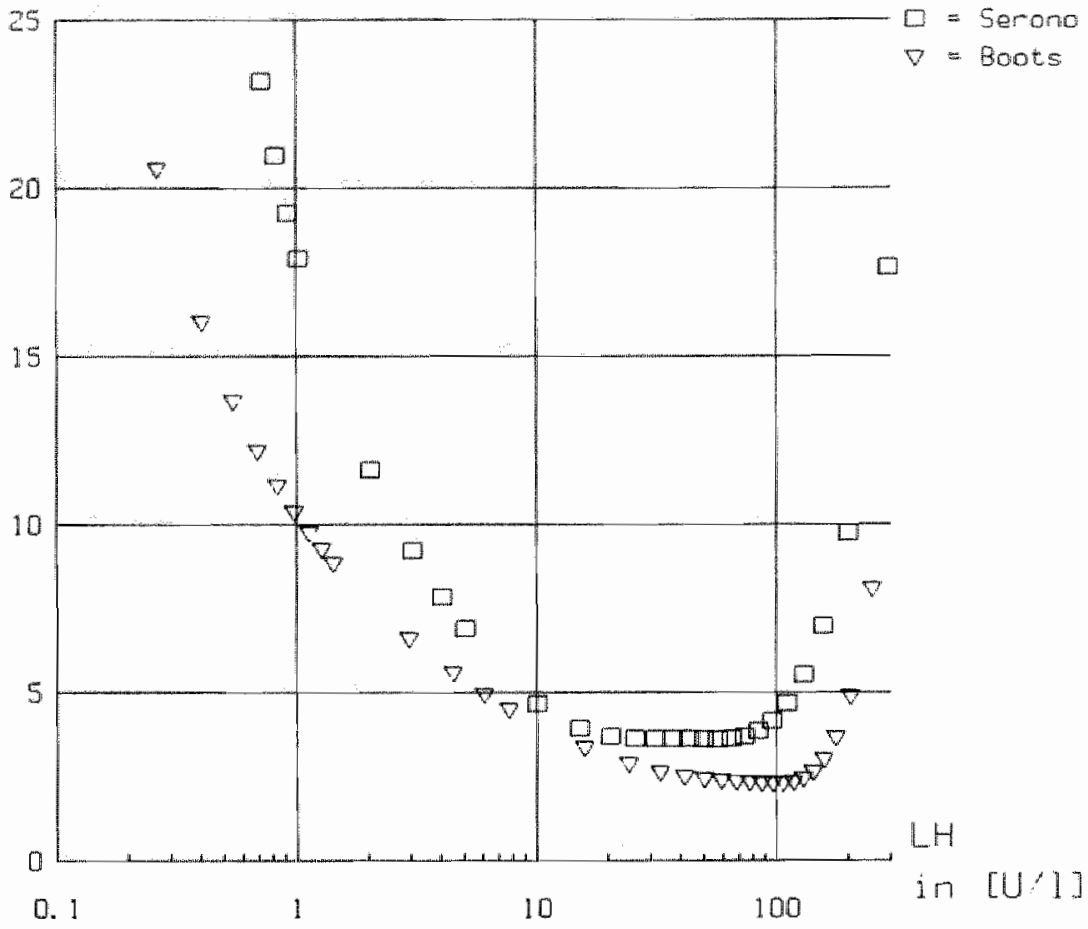

Figure 2.17: Precision protiles of LH-assays. 


\section{References.}

1: Berson S.A. Yalow R.S.: ASsay of plasma insuln in human subjects by immunological methoos. Nalure (1959) $184: 1648-1649$

2: Ekins $\mathrm{R}_{\mathrm{P}} \mathrm{P}$. The estimation of thyroxine in human plasma by an electrophoretic technique. Clin Chim Acta (1960) $5 ; 453-459$

3. Kohler $\mathrm{G}$. Milstein $\mathrm{C}$ : Continuous cutture of fused cells secreting antibooy of predefined specificity. Nature (1975) 256;445-497

4: Moody A.J., Stan M.A. Stan M.: A simple free fat cell bioassay for insulin. Horm.Melab. Res.(1974) 6;12-16

5: Dutau M.L. Pock R. Neubauer A., Catt K.J.: In vitro broassay of LH in human serum: the rat interstitaf cell testosterone (RICT) assay. J Clin.Endocrinol Melab.(1976) 42;958-969

6: Goltzmann D. Henderson B., Loveridge N.: Cytochemical bioassay of parathyrod homone.

J. Clin Inwest (1980) 65:1309-1317

7: Bangham D. R.: Immunoassays for Clinical Chemistry in: Hunter W.M., Come

JE.T.(Editors):Immunoassays for Clinical chemistry Churchill Livingsitone, Edinburgh (1983)

8: Robyn C. Meuris S., Svoboda M., Christophe J.: Heterogeneity of pituitay protem homones. In: Bizollon

Ch.A. (Editor): Monoclonal Antibodies and new trends in immunoassays. Elsevier Science Publishers.

Amsterdam (1984)

9: Vaitukaitis J.L., Ross G.T., Braunstein G.D., Raytord P.L.: Gonadotropins and their subunits. basic and clinical studies. Recent Prog.Hormone Res.(1976) 32; 289-321

10: Gewirtz R. Yalow R.S.: Ectopic ACTH production in carcinoma of the fung. J.Clin Invest.(1974)

53:1022-1032

11: Muller-Gartner H.W. Schneider C.: Chinical evaluation of tumor chavacteristics predisposing serum thyroglobulin to be undetectable in patients with differentiated thyroid cancer. Cancer 1988$) 61 ; 976-981$

12: Ginkel LA. van: Heterogeneity of human luteinizing hormone and its aloha and beta subunits. Thesis,

Riljks.universiteit Utrecht (1987)

13: Berson S.A. Yalow R.S.(Editors): Methoos in investigative and diagnostic endocinology. North-Holland Publishing Co, Amsterdam (1973)

14: Ekins R.P., Newman G.B., O'Riordan J.L.H.: Theoretical aspects of "Saturation" and radioimmunoassay. In: Hayes R.L., Goswitz F.A. Pearson Murphy B.E.: Fadioisotopes in Medicine: In viltro Studies. U.S. Atomic Energy Commission (1968)

15: Rodbard D.: Statistical Qualty Control and routine data processing for radiommunoassays and immunoradiometric assays. Clin.Chem.(1974) 20;1255-1259

16: Jackson T.M., Marshall N.J., Ekins R.P.: Optimization of immunoradionetric (fabelled antibody) assays. In: Hunter W.M., Corrie J.E.T.(Editors): Immunoassays for Clinical chemistry. Churchill Liwingstone, Edinburgh (1983); pp557-575

17: Ekins R.P.: Current concepts and future developments. In: Colwins W.P.(Ediror): Alternatwe Immunoassays. John Wiley \& Sons (1985); pp219-237

18: Ekins R.P.: The "Precision profle": Its use in Rla; assessment and design. The Ligand Quaterly (1981) $433-44$

19: Hunter W.M.: Recent Advances in radioimmunoassay and related procedures. In: Radioimmunoassay and related procedures in medicine IAEA, Vienna (4982); 5 31-18

20: Ginkel LA. van, Loeber J.G.: Heterogeneity of human htropin. Detection and identification of alpha and beta subunits. Acta Endocrinol.(1985) 110;182-192

21: Wehman F.E., Rubenstein H.A., Nisula B.C.: A sensitive convenient radioimmunoassay procedure which demonstrates that serum hTSH is suppressed below the normal range in thyrotoxic patients.

Endocrinot.Res.Commun.(1979) 6;249-245

22: Alexander W.D., Kerr D.J.; Ferguson M.M.: First line test of thyroid function. The Lancet (1984);ii $647-648$

23: Gow S.M., Elder A., Caldwell G., Bell G., Seth d., Sweeting V.M., Tolt A.D. Beckelt G.t.: An improved approach to thyroid function testing in patient's with non-thyroidal ithess. Clin. Chim. Acta (1986) 158;49-58 24: Wiersinga W.M.: The value of sensitve TSH measurements in clinical practice.

J. Endocrinol. Invest.(1986) 9/4;67.76 
25: Kalliner A, Kather G. Ljunggren J.G., Sioberg H.E. On the eficacy of highty sensitwe s-TSH methods in the diagnosis of hyperthyroidism. Opmean (1984) 29:98-101

26. Seth J., Kellett H.A., Caldwell G., Sweeting V.M., Becket G.J., Gow S.M., Toll A.D.: A sensitwe immunoradometic assay for serum thyroid simulating hormone a replacement for the thyrotophin releasing nomone test? Br.Med.4.(1984) 289;1334-1336

27: Kers D.J., Alexander W.D.: is the THH-test usually unnecessan? The Lancel (1984); it 923

28: Finlayson $J_{*}$, Sneddon W. Percy-Robb I.W: Interterence in commercial assays for thyrotropin. The Lancet (1987); $1445-446$

29: Wright J.F. Hunter WM: The sucrose layering separation: a non-centitugation system. In: Hunter W.M. Corrie J E.T.(Editors): Immunoassays for Cinical chemistry. Churchill Livingstone, Edinburgh $(1983): 170-177$

30: Wide L.: Male and liemale forms of human follicle stimulaing hormone in serum.

J.Clin.Endocrinol Metab (1982) 55,682-688 


\title{
Chapter 3.1
}

\section{Effect Of Ritodrine On Thyroid Hormone Concentrations}

\author{
P.P.C.A.Menheere, G.G.M. Essed and J.P.J.E. Sels \\ (published in Clinica Chimica Acta (1987) 165;359-364)
}

\subsubsection{Introduction}

Ritodrime (Prepar $\left.{ }^{(}\right)$, Yutopar ${ }^{(1)}$ ), a beta-2-sympathomimetic agent, is used in many countries for the prevention of preterm labour. It has been shown to prolong pregnancy after premature threat, due to a potent inhibitory effect on uterine contractions (1). The efficacy of the drug, however, is limited, mainly because of cardiovascular side effects, particularly an increase in heart firequency (2).

These effects are believed to result from an unwanted beta-1-sympathomimetic effect on the heart and a beta-2-mediated dilatory effect on the blood vessels. Another possible explanation, however, arises from a study by Murchison et al. (3), who found a significant fall in T3 levels after treatment with propranolol, a beta blocking agent, but no effect after the beta-1-selective blocker practolol, suggesting the influence upon T3 serum concentrations to be beta-2-mediated. In that case, obviously high doses of beta-2-mimetic drugs as used in tocolytic therapy, would increase the serum $T_{3}$ levels and thereby amplify chronotropic effects

Recently, Czech et al.(4) reported a sludy in which thyroid hormone levels of patients treated with fenoterol and verapamil were compared with those in healthy pregnant women. They found an increase of the $T_{3}$ concentration of $37 \%(\mathrm{p}<0.001)$ in the treated patients. This effect was thought to be due to an increase of the peripheral conversion of $T_{4}$ to $\mathrm{T}_{3}$. It is now generally accepted that $T_{3}$ is the biological active thyroid hormone in peripheral tissues. The braim and pituitary contain certain deiodinases and therefore their intracellular $T 3$ concentrations mainly depend on $T_{4}$ concentration in biood. An increased peripheral conversion of $T_{4}$ to $T_{3}$ can lead to hyperthyroid effects on tissues without causing cerebral hyperthyroidism. One of the peripheral effects of a hyperthyroid state is an enhanced hearl frequency (5). The results of Czech's investigation are difficult to interpret, since patients investigated in this trial were treated not only with fenoterol, but were given werapamil in addition.

In the present study, the influence of ritodrine on thyroid homone concentrations and thyroid stimulating hormone in blood was studied longitudinally in 17 pregnant women treated with ritodrine only. 


\subsubsection{Patients, materials and methods}

\section{Patients}

Seventeen healthy pregnant women with preterm uterine contractions who were treated with ritodrine participated in the study. No co-medication other than ferrous sulphate was allowed, no patients with prewexisting cardiovascular of thypoid disorders were included. All patients were prescribed bedrest and were given ritodrine intravenously in dose levels of $66.400 \mu \mathrm{g} / \mathrm{min}$. The dose level was the minimum supply of the drug necessary 10 suppress all uterine contractions.

Age, weight, gestational age at start of treatment and treatment dose at the moment of sampling are given in table 3.1.

Table 3. 1 Characteristics of the patients sludied.

Age (years)

Weight $(\mathrm{kg})$

Gestational age at the start of trealment (weeks, days)

Dose at $24 \mathrm{~h} \mathrm{sample} \mathrm{(u \textrm {g } / \mathrm { mim } )}$

Dose at 7 days sample (ug/min)

$\begin{array}{ll}\text { Mean } & \text { Range } \\ 28 & 21-37 \\ 69 & 54-103 \\ 31.1 & 25.0=33.6 \\ 188 & 66-400 \\ 171 & 66=400\end{array}$

\section{Materials and Methods}

Serum concentrations of TSH, T4, T3 and free-T4 were determined before tocolysis and at the second day after the start of treatment so every patient provided her own control. In patients who were treated for more than 7 days $(n=14)$ these parameters were again determined after 1 week of treatment. In those patients who had not delivered $24 \mathrm{~h}$ atter the period of treatment ( $n=10)$, samples were taken again. Except for the starting sample all blood tests were taken at 8.00 a.m. to eliminate variations due to the circadian mythm of $7 \mathrm{SH}$.

Total thyroxine $\left(\mathrm{T}_{4}\right)$, free thyroxine ( $\left.1-\mathrm{T}_{4}\right)$ and total triodothyronine $\left(\mathrm{T}_{3}\right)$ were determined by radioimmunoessay (Amersham International , Amersham. (UK), intra-assay variabilites were $<5 \%$ for the range of concentrations obtained. The inter-assay variablities were $<6 \%$. Thyroid Stimulating Hormone (TSH) was detemined by immunoradiometric assay using the protocol of the manufacturer (Boots-Celltech, Slough, UK) with slight modifications in order to reduce non specific binding. The lower detection limit was 0.04 $\mathrm{mL} / \mathrm{h}$. The intra-assay variability was $<4 \%$ over the range of concentrations obtained in this study, the inter-assay coefficient of variation less than $6 \%$. Cross-reactivity in the TSH-assay was not detectable at 50000 UHCG/l. The weight to weight ratios of the cross-reactivities of LH and FSH were 0.009 and 0.004 , respectively. 


\subsubsection{Results}

The results were analyzed by means of Student's t test for paired data. A statistically significant increase of serum $T_{3}$ concentrations $(p<0.02)$ on the second day of treatment in comparison to the pre-treatment levels was observed.

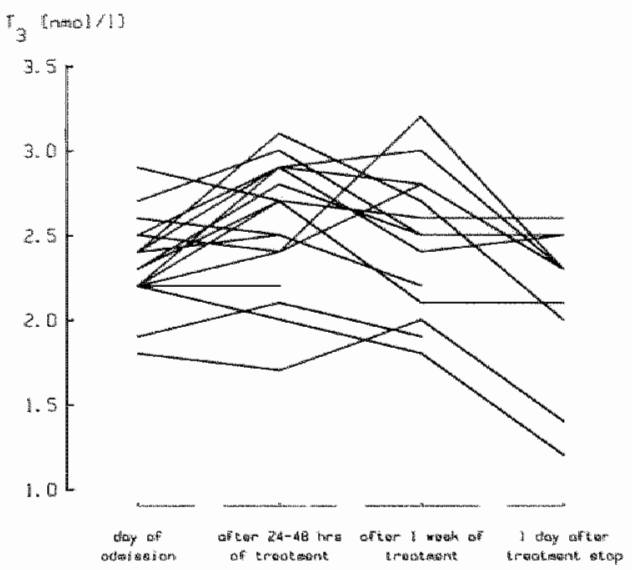

Figure 3.1: The changes of the triiodothyronine concentrations versus time. [time axis is not constant.]

Figure 3.11 shows a graphic representation of the changes in serum $T_{3}$ concentrations. On the second day of treatment, only 1 patient reached a peak value of the serum $T_{3}$ concentration of $3.1 \mathrm{nmol} / \mathrm{l}$, the upper limit of normal in our laboratory being $3.0 \mathrm{nmol} / \mathrm{l}$ However, she showed in the same samples increased TSH values (pre-treatment: $T_{3}=2.4$ $\mathrm{nmol} / \mathrm{l} ; \mathrm{TSH}=0.8 \mathrm{mU} / \mathrm{l} ;$ after 1 day: $\left.\mathrm{T}_{3}=3.1 \mathrm{nmo} / / \mathrm{l} ; \mathrm{TSH}=1.1 \mathrm{mU} / \mathrm{l}\right)$.

In the whole population the changes in total $\mathrm{T}_{4}$, free- $\mathrm{T}_{4}$ and $\mathrm{TSH}$ concentrations were statistically significant (Table 3.2). In 14 out of 17 patients, treatment was continued for more than 1 week. The initial increase in serum $T_{3}$ concentrations was followed by a small decrease after the first week of treatment. The T3 levels after 1 week did not show significant differences with pre-treatment levels. At this time, however, the decrease in free- $T_{4}$ concentration was significant $(p<0.01)$. Total thyroxine and the TSH concentrations remained at the same level.

Table 3.2 Thyroid stalus before, during and after treatment with ritodrine

\begin{tabular}{|c|c|c|c|c|c|}
\hline & n & $\begin{array}{l}1-T_{4(p m o b l l)} \\
(\text { mean } \pm s d)\end{array}$ & $\begin{array}{l}\mathrm{T} \text { (monoly) } \\
\text { (mean } \pm \mathrm{sd} \text { ) }\end{array}$ & $\begin{array}{l}\pi 3(\text { ntrolly) } \\
\text { (mean } \pm \text { sd) }\end{array}$ & $\begin{array}{l}\text { TSH(mu/l) } \\
(\text { mean } \pm \mathrm{Sd})\end{array}$ \\
\hline Before treatment & 17 & $13.3 \pm 2.6$ & $173 \pm 37$ & $2.3 \pm 0.3$ & $1.7 \pm 1.4$ \\
\hline $24-28$ h of trealment & 17 & $13.2 \pm 2.3$ & $175 \pm 36$ & $2.6 \pm 0.4^{3}$ & $1.5 \pm 1.0$ \\
\hline 1 week treatment & 14 & $12.7 \pm 2.5^{b}$ & $172 \pm 38$ & $2.5 \pm 0.4$ & $1.3 \pm 1.2$ \\
\hline After treatment & 10 & $12.6 \pm 3.4$ & $168 \pm 52$ & $1.9 \pm 0.8^{c}$ & $1.1 \pm 0.8$ \\
\hline
\end{tabular}

${ }^{a} p<0.05$ from pre-treatment levels; $\quad b p<0.01$ from pre-treatment lievels:

$c_{p<0.05}$ from pre-treatment levels and $p<0.01$ from levels during treatment. 
Ten patients did not cieliver immediately after cessation of ritodrine treatment. In these patients a significant decrease in serum $T_{3}$ levels in comparison both to treatment and pre-treatment levels was seen.

\subsubsection{Discussion}

The present study confirms the observations of Czech (4) that beta-2- sympatho mimetica increase the serum $\mathrm{T}_{3}$ concentrations during pregnancy. One of the possible explanations for this elevation could be an enhanced intra thyroidal synthesis of the hormones

However, this seems less probable since there are no significant changes in T4 or TSH concentrations.

Another explanation can be an increased peripheral conversion of $T_{4}$ to $T_{3}$. At least three different delodinase enzymes are known (6). Type $l$ is mainly situated in liver and kidney. This stimulates both the inner and outer ring deiodination $\left(T_{4} \rightarrow T_{3}, T_{4} \rightarrow r T_{3}\right.$ and $T_{3} \rightarrow T_{2}$ ). Type || deiodinase, located in brain and pituitary, stimulates the outer ring deiodination and thus the conversion of $T_{4} \rightarrow T_{3}$ and $\mathrm{r}_{3} \rightarrow 3,3 \mathrm{~T}_{2}$. Type III stimulates the deiodination of the inner ring of the thyroid hormones $\left(T_{4} \rightarrow r T_{3}\right.$ and $\left.T_{3}->3,3 T_{2}\right)$. This is found in brain, skin and placenta.

Parallel to their observation of increased serum T3 concentrations, Czech and coworkers demonstrated also an increase in reverse T3 concentrations.

Ritodrine induced stimulation of the type 1 deiodinase, therefore, could be the cause of both observations.

Stimulation of type III will lead to increased reverse $T_{3}$ and decreased $T_{3}$ concentrations, resulting in increased TSH concentrations. TSH has been assayed with a modern method, capable of detecting changes of peripheral TSH concentrations. However, we did not observe any significant change in TSH level. For this reason it is less probable that ritodrine stimulates the type $\| I$ enzyme.

The observed significant rise of the serum T3 concentration seems to be mainly the result of activation of the type / deiodinase. One of the most important side effects of ritodrine is reported to be tachycardia (7). Four times in our study we found $T_{3}$ concentrations above the upper limit of normal $(3.0 \mathrm{nmol} / \mathrm{M})$. However, only in 1 out of our 17 patients the TSH production by the pituitary was found to be suppressed. On the basis of these measurable TSH concentrations it can be concluded that these patients remained euthyroid (8-10). Thus, although there was a significant increase of the T3 serum concentration, undoubtedly contributing to the tachycardia, there are no signs for a systematic development of hyperthyroidism in patients on ritodrine therapy.

The conversion rate of $T_{4}$ to $T_{3}$ seems to change only during the beginning of the ritodrine treatment. After 1 week of treatment serum $T_{3}$ levels do not show signilicant differences with pre-treatment levels and after cessation of therapy the serum $T_{3}$ concentrations were lower than before treatment. These changes in concentrations are not in agreement with the data of Czecti et al. (4), who found increases in T3 proportional to the duration of treatment. Furthermore, the percentages of increase of $T_{3}$ concentration was $11 \%$; lower than the $37 \%$ found by Czech et al. This could be explained by the relatively high doses of the betamimetic agent with which their patients were treated.

Although limited in time, the beta-mimetic increase in serum $T_{3}$ levels provides a reason 
for restriction of the use of betamimetic drugs in hyperthyroidic patients and ofers an additional explanation for the undesirable chronotropic cardiac side-effects of this therapy.

\subsubsection{Summary}

In a clinical study of 17 pregnant women treated with ritodrine, a beta-2-sympathomimetic agent used for tocolysis, thyroid hormone status was assessed longitudinally. This was done in order to verify the hypothesis that an increase in $T_{3}$ levels could result from adrenergic stimulation, since propranolol, a beta blocking agent, has proved to decrease T3levels in man.

We have observed a significant increase in serum T3 concentrations $24-48$ h after the start of the ritodrine treatment. The changes were only temporarily since one week after the start the serum $\mathrm{T}_{3}$ concentrations did not differ significantly from the pre-treatment levels. A decrease in $T_{3}$ levels was lound after discontinuation of treatment. No significant changes were found after discontinuation of treatment. No significant changes were found in $\mathrm{T}_{4}$ and $\mathrm{TSH}$ concentrations excluding an influence in ritodrine therapy on the pituitary-thyraid axis. It was concluded that stimulation of type I cleiodinase was responsible for the changes in $T_{3}$. These beta-2-mimetic variations may explain, to a centain degree, the unwanted chronotropic cardiac side effects of ritodrine and necessitates much care in using this therapy in hyperthyroidic patients.

\section{References}

1: Wesselius-de Casparis A., Thiery M. Yo le Sian A., Baumgarten K., Brosens I., Gamissons O., Stolk J.G., Villier W.: Results of a double-blind, multicentre study with ritodrine in preterm labor. Br.Med.J. (1971) $3 \cdot 144-147$

2. Eskes T.K.A.B., Essed G.G.M.: Inhibition of wienne contractihy with beta-mimetic arugs. In: Keirse M.J.N.C., Anderson A.B.M., Bennebroek Gravenhorst a, eds: Human partirition, Universily Press, The Hague (1979); 165.187

3: Murchison L.E., Bewsher P. D. Chester M.L. Ferrier W.R.: Comparison of propranolol and practolot in the management of hyperthyroidism. Br.J.Clin.Pharmacol(1976) 3,273-277

4: Czech W., Draw-Kaniewska d. Pisarek-Meidzinska J., Kuczynska-Sicinska J.: The eftect of Phenoterol and Verapamil on thyroid hormone concentrations in pregnant women. NucCornpact (1983) 14,224

5: Barnes C.: Medical disorders in obstetric practice. Oxford:Blackwell Scientific Pubi. (1976)

6: Leonard J.L., Visser T.J.: Biochemistry of deiodination. In: Henneman G. "ed.: Thyroid hormone metabolism. Marcel Dekker, New York (1986): 189

7: Tolino A., De Concilis B., Montemagno U.: Thyroid hormones in the human pregnancy. Acta Obstet.Gynecol.Scand.(1985) 64;557-559

8. Seth J.A.: A sensitive immunoradiometric as say for serum thyroid stimulating homone. Bril.Med J. (1984) $289 ; 1334-1336$

9: Alexander W. D.: First ine test of thyrojid function. The Lancet (1984):647

10: Wiersinga W.M.: The value of sensitwe TSH measurements in clinical practice. Endocrinol.Invest. (1986) $9,67 \cdot 76$ 


\title{
Chapter 3.2
}

\section{Thyroid hormone status during the follicular and luteal phase of the menstrual cycle in eumenorrheic women.}

The importance of highly sensitive TSH-assays.

\author{
P.P.C.A.Menheere and H.A. Keizer
}

\subsubsection{Introduction}

In women, body temperature in the luteal phase of a normal ovulatory menstrual cycle is raised by 0.2 to $0.5^{\circ} \mathrm{C}(1)$, although the underlying mechanism is not clear (2). it may be due to a direct effect of progesterone $(P)$ on the central thermoregulatory system, or to changes in the concentration of free thyroid hormones. It is now well accepted that thyroid hormone increases the number and the respiratory capacity of the mitochondria $(3,4)$. Subsequent stimulation of the utilization of ATP (by induction of RNA and protein synthesis) is thought to be the mechanism behind the thermogenic action of thyroid hormones. One of the induced cellular processes that increases ATP consumption is the active transmembrane $\mathrm{Na}^{+}$transport (5).

The quantity of heat that is liberated in these processes maintains the basal body temperature. Slight changes in free thyroid hormones possibly induce minor changes in the basal metabolic rate resulting in a slight change in body temperature. However, actual RIA techniques are not sensitive enough to demonstrate such differences in the free thyroid hormones concentrations.

Since TSH and both $T_{4}$ and $T_{3}$ are involved in a negative feedback system slight changes in concentrations of thyroid hormones are reflected in changes in TSH concentration. Unllike RIA techniques, the modern IRMA sandwich assays for TSH offer the required sensitivity to demonstrate slight changes in thyroid hormone status possibly associated with the increase in basal metabolic rate.

The purpose of this study was to investigate whether there is a relationship between thyroid hormone status and phase of the menstrual cycle by using sensitive IRMA TSH assay.

\subsubsection{Subjects, materials and methods.}

\section{Subjects}

Six healthy female subjects with a mean age of 22.5 years (s.d.: 1.8 years) participated in this study after written informed consent. All subjects had a regular menstrual cycle. To determine normality of the cycle, serial blood samples (each other day) were taken during the experimental cycle in which progesterone (P), luteinizing hormone (LH), follicle stimulating hormone (FSH) and estradiol were measured (data not shown). For the present experiment, blood was taken in the follicular phase between the seventh and tenth day and in the luteal phase of the menstrual cycle between the twentieth and twenty-fifth 
day. To avoid differences due to the circadian rhythm in the TSH concentrations all venapunctures were performed between 8.00 and 8.30 hours a.m.

\section{Materials and methods}

Plasma concentrations of progesterone have been determined by a no-extraction, direct solid-phase ${ }^{125}$ I radioimmunoassay with the Coat-A-Count assay of DPC (Diagnostic Products Corporation, Los Angelos, U.S.A. Intra-assay variabilities are less than $6 \%$ for the concentration range of 1.0 to $90 \mathrm{nmol} / \mathrm{h}$.

The serum TSH concentrations have been determined with the immunoradiometricassay (IRMA) of Boots-Celltech (Slough, U.K.) using the protocol of the manufacturer with slight modifications in order to reduce non specific binding. It is a sandwich type assay using two monoclonal antibodies. The first antibody is coupled to a solid phase and is directed to the beta subunit of the TSH molecule; the second antibody is directed to the whole molecule and has been labeled with ${ }^{125}$. The lower detection limit is $0.04 \mathrm{mU} / \mathrm{l}$. Figure 2.6 (TSH-IRMA) shows the intra-assay variabilities calculated from all duplicates within the assay (standards, control samples and unknowns). In the range of concentrations obtained in this study, the coefficient of variation was less than $6 \%$. Cross-reactivity in the TSH assay was not detectable at $50.000 \mathrm{U} \mathrm{HCG/l}$. The weight to weight ratios of the cross-reactivities of $\mathrm{LH}$ and FSH were 0.009 and 0.004 , respectively.

\section{Statistics}

Standards, controls and samples have been analyzed in duplicate. For TSH concentrations the $95 \%$ confidence limits have been calculated from the 5 parameter logistic-log transformation of the calibration curve. The Wilcoxon signed rank test has been used to test the significance of differences between the two samples within each menstrual cycle.

\subsubsection{Results}

In table 3.3 the individual data of the TSH concentrations in the follicular and luteal phase are compiled. In the luteal phase all TSH-values but one were lower than in the follicular phase.

The $95 \%$ confidence intervals of each result in the pair of TSH-data for each of the subjects 1 to 4 from the table did not overlap.

Although subject 5 had a decreasing TSH concentration the confidence intervals showed overlap.

The sixth subject had an (analytically non significant) increasing TSH concentration. From the progesterone concentration it can be deduced that she had an anovulatory cycle.

\subsubsection{Disciussion}

The superiority of sensitive TSH-IRMA assays in comparison to conventional RIA techniques $(6,7,8)$ is generally accepted. The improved precision and sensitivilies allow detection of small changes in TSH concentration reflecting variations in thyroid hormone status. 
Table 3.3. Individual data of TSH and Progeraterone (P) concentrations in the follicular (foll) and luteat phase of menstrual oycte.

$\begin{array}{lllll}\text { Subject } & \begin{array}{l}\text { TSH foll } \\ \mathrm{mU} / \mathrm{I}\end{array} & \begin{array}{l}\text { TSHiveal } \\ \mathrm{mU} / \mathrm{l}\end{array} & \begin{array}{l}\text { Proll } \\ \text { nmol/l }\end{array} & \begin{array}{l}\text { Pluteal } \\ \text { nmol/ }\end{array} \\ 1 & 1.47 & 1.06 & 1.0 & 54 \\ 2 & 2.77 & 2.18 & 1.3 & 65 \\ 3 & 3.75 & 3.04 & 0.5 & 32 \\ 4 & 4.73 & 2.27 & 2.4 & 54 \\ 5 & 1.70 & 1.65 & 0.7 & 40 \\ 6 & 1.31 & 1.37 & 0.9 & 1.7 \\ & & & & \end{array}$

From our data that should be considered preliminary, we conclude that TSH concentrations are significantly lower in the luteal phase compared to the follicular phase. A. lower TSH concentration can be the result of various conditions. Firstly, the set-point of the hypothalamic-pituitary-thyroid axis can be changed in order to lower the supply with thyroid hormones. Since the half-life of thyroxine is about eight days in euthyroidic subjects, while the luteal phase longs for about 12-15 days, this explanation seems less likely. (Such mechanism would be inefficient.) Secondly, the lower TSH concentration may reflect an increased thyroid hormone status. In this case, the TSH concentration obeys the mechanisms of the negative feedback system.

Displacement of thyroid hormones from binding proteins by the increased progesterone concentration in the luteal phase is an attractive hypothesis. This would result in an increase of especially the free fraction of the hormones. It is known, that biologic activity is determined only by the unbound fraction of the hormones. Therefore, displacement would result in increased biological activity of the thyroid hormones. In consequence, due to the accompanying stimulation of cellular processes, body temperature will raise. It is our opinion that the differences in the free fractions of $T_{4}$ or $T_{3}$ can not be measured with the actual available assays, In fact, the changes in TSH concentration correspond only to minor changes in the free hormone concentrations. If these latter could be detected, they would be insignificant since the changes in concentration are expected to be of the same order of maginitude as the intra-assay variation. The last possible explanation to the lower TSH concentration may be the concentration of prolactin in the luteal phase. This hormone is increased during the huteal phase in comparison to the follicular phase. Prolactin is known to influence dopaminergic pathways. while dopamine is an potent inhibitor of TSH-release. If this would be the correct explanation of the observed lowering of the TSH concentration, there should be a decrease in the free fractions of the thyroid hormones. As described above, this can not be detected with the actual assays. Furthermore, the increase in bodytemperature may be caused by a direct effect of progesterone upon the thermoregulating centre within the central nervous system as well. However, even in this case the thyraid hormones must be involved, since no other 
mechanisms are known to raise body temperature.

This leads to a conflicting situation: thyroid hormones should decrease due to the lower TSH and should increase to raise bodytemperature.

In conclusion, we suppose the displacement of thyroid hormonas from the binding proteins by progesterone, resulting in increased free thyroid hormone levels, to be the most likely explanation for the observed phenomenon of lower TSH concentrations during luteal phase. However, it will be necessary to study simultaneously the behaviour of TSH and Prolactin both assayed with sensitive, specific methods in order to procure a better insight in this part of thuman physiology.

\section{References}

1: World Health Organization: Biology of fertity control by periodic abstinence. WHO Tech:Rep. Ser. $(1967) ; 360$

2: Lipton J.M.: Thermoregulation in Pathological States. In: Smitzer A, Eberhart A.C., eds. Heal Transler in Medicine and Biology, Volume 1. Plenum Publishing Corporation (1984);79-105

3: Douglas J.E: Thyroxine-induced atterations in the fine structure of rat liver cells. Johns Hopkins Med.J.(196.4) 14:253

4: Gross N.J.: Control of mitochondrial tumower under the influence of thyroid hormone. J.Cell Biol. (1971):48:29

5: Smith T.J., Edelman I.S: The rote of sodium transport in thyroid thermogenesis. Fed.Proc.(1979) 38,2150. 2153

6: Seth J., Kellett H.A., Caldwell G. Sweeting W.M. Beckett G.J., Gow S.M., Tolt A.D.: A sensinive inmunoradiometric assay for serum thyroid stmulating hormone: replacement for the thyrotrophin releasing hormone test? Bril.Med.J.(1984) 289:1334-1336

7: Martino E., Bambini G. Bartalená L., Mammoli C. Aghini-Lombardi F. Bashieri L., Pinchera A.: Human serum thyrotrophin measurement by utrasensitve immunoradiometric assay as a first fine test in the evaluation of thyroid function. Clin. Endocrinal (1986) $24 ; 141.148$

8: Wiersinga W.M.: The value of sensitive TSH measurements in clinical practice. Endocrinol. Invest.(1986) $9 ; 67-76$ 


\title{
Chapter 3.3
}

\section{End organ responses, clinical judgement or biochemical control of} thyroxine replacement therapy in hypothyroidism?

\author{
P.P.C.A.Menheere
}

\subsubsection{Introduction}

In patients recelving replacement therapy for hypothyroidism, the balance between (subclinical) thypo-and hyperthyroid states is easily disturbed. Between these states there is a continuum and during transition of one state onto the other clinical signs and conditions of dysthyroidism are very difficult to recognize and to differentiate from the euthyroid patient.

The effectiveness of thyroxine replacement therapy during treatment for hypothyroidism is usually judged by estimations of the serum concentrations of thyroxine or free thyroxine and thyroid stimulating hormone. It is generally accepted that changes in these hormone concentrations are detectable before any alteration in the clinical state. In some cases, however, there is a need for an independent assessment of chinical status. For example. heart rate and urinary sodium excretion are influenced by onlly slight alterations of thyroid hormone concentrations (1). Particularly in patients with an impaired cardiac function such changes may represent a serious risk.

Several methods are available to quantify clinical or metabolic indices of the thyroid state. The Wayne-index is one of the oldest ones(2). Other metabolic indices are the basal metabolic rate, systolic time interval, achilles tendon reflex time and certain chemical parameters like cholesterol, enzyme activities and sex-hormone-binding-gllobulin (SHBG). Of these, the measurement of systolic time intervals by simultaneous registration of an echocardiogram and an electrocardiogram enjoyed much attention in the literature $(3,4,5,6)$.

In fact, a slight increase of thyroxine within the normal reference range may demonstrate subcinical signs of thyrotoxicosis by a reduced ratio of the pre-ejection-period versus the left-ventricular-ejection-time ( $P E P / L V E T$ ). TSH concentrations estimated by conventional methods may remain nomal in this situation and the response of TSH to TRH within the normal limils (especially if nol corrected lor sex and age). T3, which is the biologic active hormone, can be normal even in the presence of evident signs of thyrotoxicosis.

For this reason warious authors recommend to monitor patients being substituted for hypothyroidism by measuring PEP/LVET ratios in addition to the determinalion of thyroid hormones and TSH.

Recently, sensitive assays became available for the estimation of TSH (7,8). The sensitivity of these assays is reduced from about $1 \mathrm{mU} / \mathrm{I}$ (WHO 80/558) to better than $0.05 \mathrm{mU} / \mathrm{l}$ and enable true separation of hyperthyroid from euthyroid patients $(9,10)$. The relationship between TSH concentration determined by a sensitive method and other biochemical parameters during the introduction of suppletion therapy and under stable substitution therapy has been described by Gow et al.(11). 
However, they did not take into consideration functional parameters as the PEP/LVET ratlo to judge the degree of substitulion.

The aim of our investigation was to establish the value of sensitive TSH assays in relation to the assessment of functional and biochemical parameters in clinically euthyroid patients receiving thyroxine replacement.

\subsubsection{Patients, Materials and Methods}

\section{Patients}

Twenty five patients with primary hypothyroidism were selected for this sludy. For inclusion in the study, the patients had to be treated with l-thyroxine for at least 1 year, without any dose adjustment within six months prior to the study.

One male and 24 females participated in the study. Their mean age was 54.2 years (range 24.1-71.9 years). All patients were considered clinically euthyroid, based on the absence of standard signs and symptoms suggestive for hypo-or hyperthyroidism. The mean daily dose $( \pm s . d$.) of l-thyroxine taken by these patients was $130 \pm 37 \mathrm{mg}$. None of the patients included used any other drug or suffered from any other disease known to interfere with the clinical or biochemical observations made in thyroid disease or during its treatment. In particular, patients with high blood pressure and/or heart disease were excluded from the study.

\section{Materials and Methods}

Total thyroxine $\left(T_{4}\right)$, free thyroxine ( $\left.f-T 4\right)$ and total triodothyronine $\left(T_{3}\right)$ were determined by radioimmunoassay (Amersham Internationals, Amersham U.K.). Inter-assay variabilities were $6 \%$ for the range of concentrations of $60-300 \mathrm{nmol} / \mathrm{A}$ for $\mathrm{T}_{4}, 5-50 \mathrm{pmol} / \mathrm{i}$ for $\mathrm{t}-\mathrm{T}_{4}$ and $0.8-4.0 \mathrm{nmol} / \mathrm{l}$ for $\mathrm{T}_{3}$.

Thyroid stimulating hormone (TSH) was determined by immunoradiometricassay (IRMA) (Boots “Celltech, Slough U.K.). The lower detection limit was $0.04 \mathrm{mU} / \mathrm{l}$. The inter assay variability was better than $4 \%$ over the range of 0.5 to $200 \mathrm{mU} / \mathrm{l}$ and lower than $10 \%$ over the range of $0.1100 .5 \mathrm{mU} / \mathrm{l}$. The assay is calibrated against the international reference preparation MRC $80 / 558$.

Sex hormone binding globulin (SHBG) was determined by an immunoradliometricassay available from Farmos (Farmos Diagnostica, Oulunsalo, Finland). The interassay variability was better than $3 \%$ over the complete range of clinical interest $(10-100$ nmol/l, see figure 3.2).

The pre-ejection-period (PEP) and left-ventricular-ejection-time (LVET) were determined by dopplerechocardiography. The reference interval in healthy persons for the PEP/LVET ratio is $0.27-0.39$. In hypothyroid patients a ratio of $0.39-0.65$ is found and in hyperthyroid patients a ratio of $0.18-0.34$. 
๘. W.

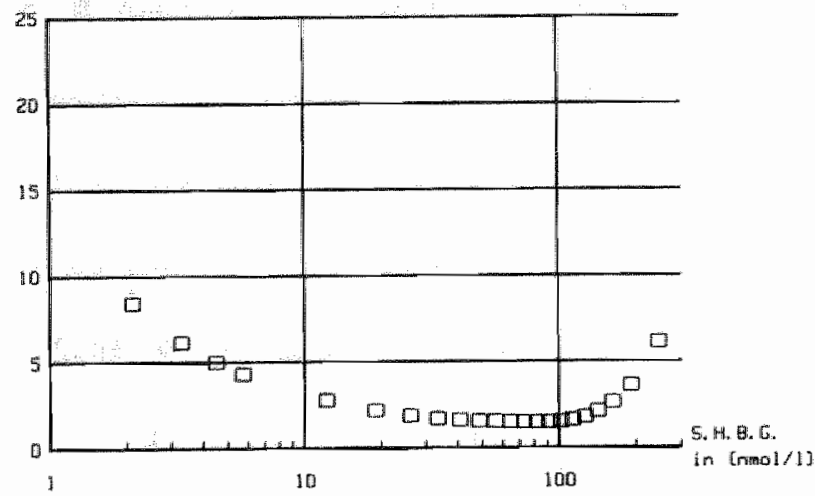

ligutur 32: Precision prollile of SHBG assay.

\subsubsection{Results}

In table 3.4, the median, the minimum and maximum values of $\mathrm{TSH}_{3} T_{4}, f-\mathrm{T}_{4}, T_{3}, \mathrm{SHBG}$ and PEP/LVET are shown.

Table 3.4: Median, minimum and maximum values of TSH, $T_{4}, 1-T_{4}, T_{3}$, SHBG and PEP/LVET.

$\begin{array}{llccc} & n & \text { Medium } & \text { Minimum } & \text { Maximum } \\ \text { TSH } & 25 & 0.8 & 0.06 & 17.8 \\ f-T_{4} & 24 & 20.6 & 13.9 & 31.7 \\ T_{4} & 24 & 133 & 90.0 & 187.0 \\ T_{3} & 23 & 1.4 & 1.10 & 2.20 \\ \text { SHBG } & 23 & 47 & 17.0 & 190.0 \\ \text { PEP/LVET } & 25 & 0.35 & 0.17 & 0.50\end{array}$

The individual values for each of the variables are plotted in figure 3.3. The reference ranges have been plotted in the same figure. The symbols used correspond to a hypo-. hyper-or euthyroid status according to the level of the TSH concentration within the same patient: hyperthyroid: $\mathrm{TSH}<0.4 \mathrm{mU} / \mathrm{l}$ : hypothyroid: $\mathrm{TSH}>3.5 \mathrm{mU} / \mathrm{l}$ and euthyroid: $0.4<=\mathrm{TSH}<=3.5 \mathrm{mU} / \mathrm{M}$. 


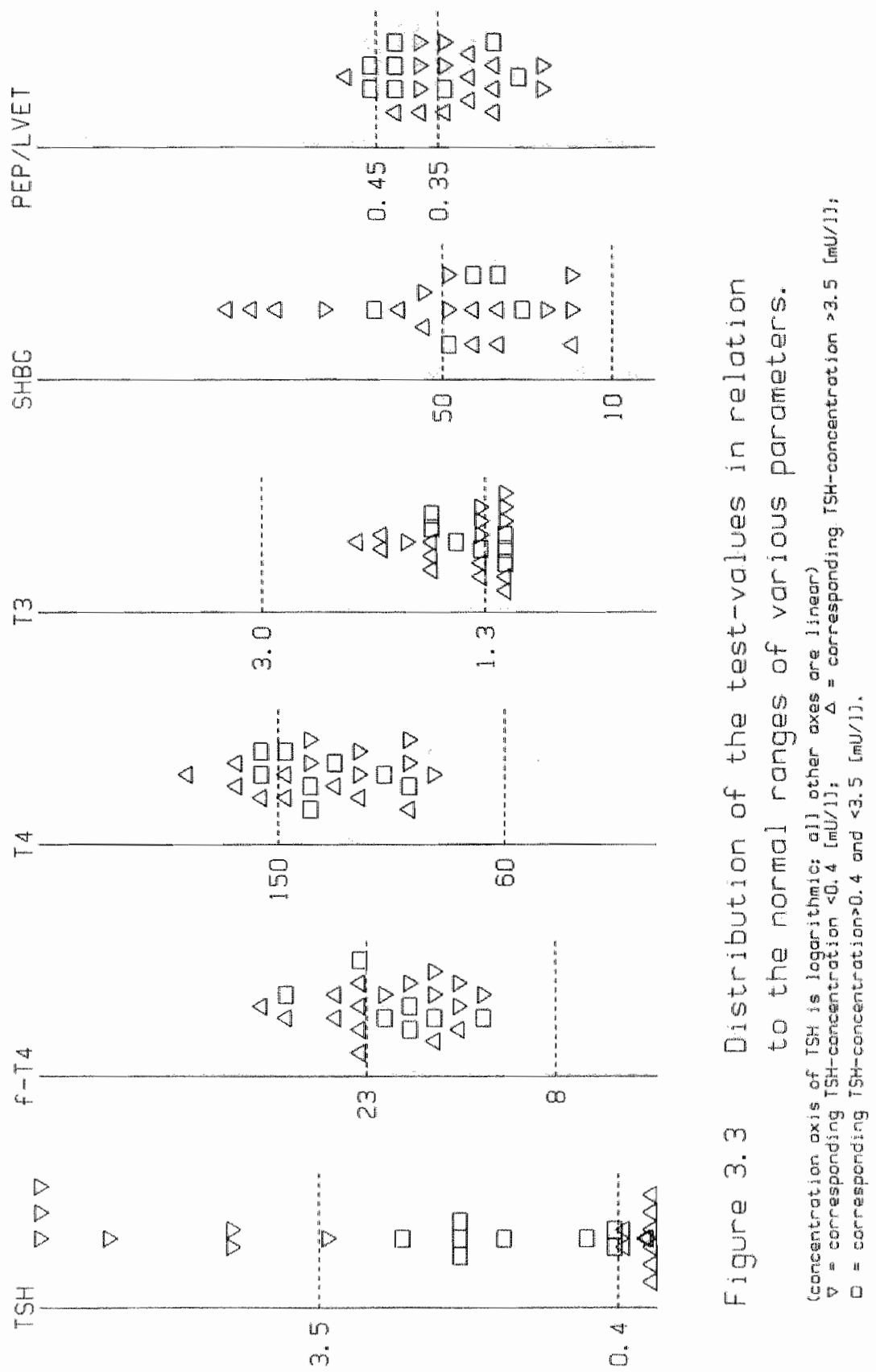




\subsubsection{Discussion}

On the basis of the papers of Crowly et al. (3), Yamauchi et all.(4) and Vora et al (5) a positive correlation may be expected between biochernical and functional parameters with regard to the diagnosis of under-or oversuppletion during 1 -thyroxine therapy. However, all these studies were characterized by a longitudinal design: Crowly et al. followed patients with severe hypothyroidism during establishment of the euthyroid state. During this period the TSH concentrations showed a significantly positive correlation ( $r=0.59)$ with the values of the PEP/LVET ratios. Oversuppletion with 200 or $300 \mu \mathrm{g}$-thyroxine per day resulted in decreased PEP/LVET ratios.

Yamauchi et al (4) performed a longitudinal study as well. LVET/PEP agreed well with serum TSH. These authors concluded that the heart is likely more sensitive to thyroid hormones than the thyrotrophic ceils in the pituitary, since $40 \%$ of the patients had an increased LVET/PEP ratios in combination with normal serum TSH levels. However, the assay for thyrotrophin applied by these authors was of a non-sensitive type and therefore a discrimination between hyper-and euthyroid states on basis of the TSH concentrations was impossible.

The sensitivity of our TSH-assay is better than $0.05 \mathrm{mU} / 1$. In non treated patients, the nosologic sensitivity of this assay to recognize dysthyroidism is better than $97 \%(12)$. However, in our study which had a transversal character rather than a longitudinal, no correlation is found between any of the biochemical parameters and the two functional parameters, PEP/LVET and SHBG.

All our patients were clinically euthyroid. Fourteen patients (58\%) appeared to have normal $f_{-} T_{4}$ concentrations, while even 16 patients $(67 \%)$ had normal total $T_{4}$. Oniy 8 out of 25 patients ( $32 \%$ ) had normal TSH concentrations. The same number of patients had normal PEP/LVET ratios. Hence, if the clinical picture of the patient is considered to be the 'golden" reference, f-T $T_{4}$ and $T_{4}$ are better markers for euthyroidism than TSH or PEP/LVET. However, the reference values used are those of healthy persons and it is known that both the $f_{-} T_{4}$ and $T_{4}$ concentrations are higher in hypothyroid patients being supplemented with l-thyroxine, due to the absence of the thyroidal production of $T_{3}$.

Furthermore, subclinical states of hypo- and hyperthyroidism have to be considered. Biochemically, subclinical hyperthyroidism is defined as a lowered TSH concentration accompanied by normal $T_{x}$ hormones concentrations and a blunted TSH-response to TRH. Subclinical hypothyroidism is defined as a (slightly) elevated TSH with normal $T_{x}$ hormones concentrations and an increased reaction of TSH to TRH.

In literature, there is an increasing interest in the subclinical states of dysthyroidism (6). Until recently, if recognized, most authors accepted these syndromes without any therapeutic consequence. Some authors, however, suggest early diagnosis and treaiment of dysthyroidism to be important. In both the hypothyroid and the hyperthyroid state, the heart seems to be the peripheral organ, which is the most sensitive to changes in thyroid hormone concentrations. Consequently, the PEP/LVET ratio would be a good parameter for monitoring replacement therapy, but it must be realized that this ratio is sensitive to other changes in metabolism as well and in particular to changes in the oxygen supply of the heart (17).

It is known that, in most patients with subclinical hypothyroidism cardiac systolic time 
intervals change in a significant way and the patients benefit from treatment with 1-thyroxine (13). Hyperthyroidism can be the cause of heart diseases (14). Cardiovascular symptoms are present and especially in the elderly dominate the clinical picture. Since most cardiac abnormalities return to normal after the euthyroid state has been reached. treatment might be beneficial. In this context, even subclinical hypo- and hyperthyroidism may be important with regard to possible adaptions in the replacement therapy. Subclinical dysthyroidism might be defined by a TSH concentration outside the normal range without clinical signs of hypo- or hyperthyroidism. This detinition supposes a normal hypothalamic-pituitary axis able to respond to hypo- or hyperthyroxinemia by changing the production rate of TSH. The thyrotrophic cells of the anterior pituitary are known to be very sensitive to minor changes in circulating thyroid hormones. Slight alterations in these hormone concentrations frequently occur within their normal reference ranges. The accompanying changes in TSH concentration are numerical an amplification of the changes in the thyroid hormone concentrations. Consequently, (although we do not have expirimental proves) we suppose the monitoring of the thyroxine replacement therapy in patients with primary hypothyroidism is more precise when measuring TSH instead of $T_{4}$, (free-) $T_{4}$ or $T_{3}$.

We agree with Fraser and co-authors (15) that important savings can be made by stopping measurement of (free-) $T_{4}$ and $T_{3}$ in primary hypothyroidism. However, we do not follow their recommendations to rely only upon clinical features. As long as there is no better index for dysthyroidism we believe that measurement of TSH by a sensitive IRMA-like method and subsequent adaption of the replacement dose can be of great benefit to patients. We suggest to extend the conclusion of Toft et al. (16) that in 'cases of discrepancy of biochemical test and clinical judgement, the biochemical tests must decide upon the clinical action' in such a way that minor deviations from normal of the TSH concentration should result in therapeutical consequences by increasing or decreasing l-thyroxine doses. This is in accordance with the conclusion of Utiger (18), who stated in a recent review article that 'the TSH-first strategy is clearly better in the assessment of patients receiving thyroxine therapy'.

\subsubsection{Conclusion}

Although the PEP/LVET ratio has proven to be useful in longitudinal studies during the initial treatment of dysthyroidism, this parameter is of little value in the assessment of a transversal sample of the population with primary hypothyroidism. The correction of subclinical states of hypo-or hyperthyroidism as can be the result of under-or overdosage of I-thyroxine preferentially should be done according to the results of the estimation of TSH with a sensitive IRMA or IRMA-like method. 


\section{References}

1. G.M.Cell, W.S.A.Sawers, J.C.Fortar, A.Doig and A. D Tott: The eftect of mino increments in plasma

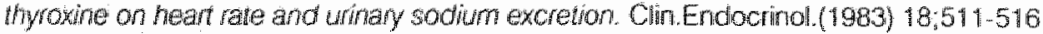

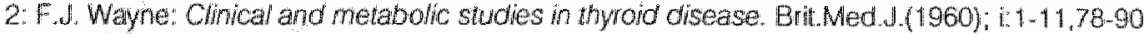

3: W. F.Crowley Ir, E. C. Ridgway, E.W.Bough, G.S.Francis, G.H.Danuls, I.A.Kourides, G.S.Myers, F.Malool Non inwaswe evaluation of cardlac function in hypothyoidism. New Eng J.Med (1977) 296;1\%6

4. K. Yamauchi, N. Takasul, K.Ichikawa, T.Yamada, T.Aizawa: Effects of long term treatment with thyroxine on pitultary TSH secretion and heart action in patients with hypenthyroidism. Acta Endocrinologica (1984) $107,218 \cdot 224$

5: J Vora, B.P.OMalley, S.Petersen, A.MoCullough, F.D.Rosenthal, D.B. Barnett: Reversible abnomalifes of imyocardia/ relaxation in hypothyroidism. J.Clin Endocrinot.Metab. (1985) 61:269-272

6: A.Nicolini, G.Cini, G.lenval, A.Carpi: Cardiac pertomance in clinical and subchinical thyrowd dystunctions: prelinhitury study. IN: "Thyroid Function and Dystunction: Modern Concepts" Dy A.Carpi and A. Nicolini, editors: ETS editrice, PISA (1986) P221-231

7: J.Seth, H. A. Kellett, G.Caldwell, V.M.Sweeting, G.J.Beckell, S.M.Gow, A.D.Toft: A sensitve

immunoradiometric assay for serum thyroid stimulating formone: a replacement for the thyrotropin reteasing hormone tes? Brit.Med.d.(1984) 289;1334-1336

8: E.Mantino, G.Bamibini, L.Bartalena, C.Mammoli, F.AghiniLombandi, L.Baschien, A.Pinchera: Human serum thyrotrophin measurements by ultasensitive mmunoradiametric as say as a first line vest in the evaluation of thyroid function. Clin. Endocrinot.(1986) 24;141-148

9: J.S.Malter, S.E.Manott, G.P.Knee, D.B.P.Goodman. J.F.Strauss: Identification of hypenthyroid patiens by means of a sensitive assay for thyrotopin, Chin. Chem.(1985) 31,642.644

10: G.Caldwell, S.M.Gow, V.W.Sweeting, H.A.Kellet, G. .Beckett, J.Seth, A.D. Tott: A new stralegy for thyroid function testing. The Lancet (1985) $1117-1119$

11: S.M.Gow, G.Caldwell, A.D.Tolt, J.Seth. A.J.Hussey, V.M.Sweeting and G.J.Beckett: Relationship between Pitutary and other Target Organ Responsiveness in Hypothyroid Patvents Receving Thyroxine Replacement. J.Clin Endocrinol.Metab.(1987) 64;364-370

12: W.M.Wiersinga: The value of sensitwe TSH measurements in clinical practice. Endocrinollinvest. (1986) $9 ; 67-76$

13: E.C.Ridgeway, D.S.Cooper: Subclinical Hypothyroidism. IN: "Thyroid Function and Dysfunction: Modern Concepts" by A. Carpi and A. Nicolini, editors; ETS editrice, PISA (1986) p297-307

14: J.C.Forfor, G.C.Caldwell: Hyperthyroid heart disease. IN: "Clinics in Endocrinology and Metabolism". Volume 14: Hyperthyroidism by A.D. Toft, editor; Saunders London (1985) p 491-508

15: W.D.Fraser, E.M.Biggari, D.St.J. O'Reilley, H.W.Gray. J.H.McKillop, J.A. Thomson: Are biochenical tests of thyroid function of any value in monitoring patients receiving thysoxine replacement therapy?

Brit.Med.J.(1986) 293;808-810

16: A. D. Tott: Thyroxhe replacement treatment: clinical judgment or biochemical control ? Brit. Med.J.(1985) $291,233-234$

17:Evers J.L.H.: The cardac pre-ejection-period during prenatal We Thesis Katholieke Universiteit Nijmegen $(1978)$

18:A.D. Utigen:Thyrotropin Measurements: Past, Present, and Future. Editorial, Mayo Cin. Proc. (1978) $03: 1053-1056$ 


\section{Chapter 4:}

\section{Introduction to Pulsatility, Pulse Detection and Changes in Pulsatility due to Exercise.}

\subsection{Endocrine Rhythms}

Living organisms (animals but probably also most plants) are subject to periodic changes. Such changes may be induced by climatologic conditions or by the day-to-night variation. In animals these variations are mainly influenced by the nervous system $(1-4)$.

Periodic variations can be of the 'free-running' type; i.e. independent from the environment an intrinsic mechanism within the organism is responsible for the changes. Some of these free-running regulatory mechanisms are synchronized by external sicnals, such as changes from day to night, or temperature variations in seasons.

Many endogenous rhythms have a intrinsic period of a little more than 24 hours. They are synchronized by external sources to exactly 24 hours. Rhythms with a periodicity of about 24 hours are called circadian. If the periodicity occurs more than once within a day the thythm is said to be ultradian.

Other periodic changes are known to have clearly lower frequencies: the human menstrual cycle is approximately 28 days $(21-35)$, while some plants and animals have cycles of 1 or even 3 years.

Most endocrine functions are characterized by circadian rhythms, whereas the secretion pattern of at least some of the pituitary hormones show an ultradian rhythm as well. Knowledge of the exact character of endocrine rhythms is necessary in order to determine the most appropriate moment for blood sampling and to interpret correcitly the results of various hormone measurements. Furthermore, such knowledge is indispensable in the development and the performance of dynamic function tests of the endocrine axes, that enables clear differentiation of any lesion of one of the axes.

In humans, the pituitary is an important endocrine organ, secreting various hormones all having crucial biological functions. The secretions are regulated by releasing hormones coming from the hypothalamus, inhibiting hormones like dopamine and inlibin and by the hormones that are produced by the target organs themselves. The secretion pattern of most pituitary hormones shows a circadian thythm, whereas most of them are also secreted in brief, fairly regular pulses.

The short time fluctuations are suggested to be the driving forces of biological processes in target organs (25), although for some pituitary hormones, like $\mathrm{LH}$ and $\mathrm{GH}$, it is now well established that continuous application of exogenous hormone leads to downregulation of the receptors and consequently target cell insensitivity $(5,6)$. Episadic secretion of the hormones might avoid such down regulation of receptor response and is even supposed to be necessary in order to maintain the normal blood concentration of the hormone produced by the target cell (55). This may be explained by stimulation of the receptor synthesis during periods of low concentration in stimulating hormone. 
Subsequent increase in the concentration of the stimulating hormone leads to an increased receptor response.

One of the merits of modern medicine is the possibility to deal with problems that cause discomfort rather than being the source of morbidity or even mortality. A characteristic example is intertility, the treatment of which became possible by profound knowledge of physiology and anatomy of the reproductive system. Especially here, it is evident that measurement of the plasma concentrations of the hormones produced by the gonads does not procure a profound insight into the mechanism of the dysfunction of the gonads Such concentrations can only be compared to established reference concentrations. However, it must be realized that the reference values rellect inter-individual variances, that are much larger than the intra-individual variances (7). If available, the measured homone concentration can be compared to previously determined results, but in that case. knowledge must be present about the behaviour of the hormone concentration in time, especially for the given clinical condition. Comparison of the plasma hormone concentration with the patients own 'set.point' might lead to a correct conclusion about rypo- or hyperstimulation.

Since a particular hormone concentration is the result of periodic stimulation of the target-tissue responsibie for the hormone production, the setpoint of the target organ can be wisualized by the pulse frequency and the pulse amplitude of the stimulating hormone As is generally the case in cybernetics, the numerical differences in puise amplitude and frequency of the concentrations of the stimulating hormones between individuals will be smaller than to the interindividual changes in the concentrations of the target cell hormones. Sometimes, the presence or the absence of episodic release of stimulating hormones (pulses) would already be important in certain climical conditions like delayed on precocious puberty (56).

However, the techniques of pulse detection and pulse characterization in terms of amplitude and frequency on one hand and target cel response on the other hand are in an early state of development (8). Hence, it is not yet possible to state anything with reasonable certainty about the relation between $L H$ and FSH peak amplitude and peak frequency and a normal response of the gonads hereupon. In addition, we are not even informed about normal responses of hormone pulsatility on normal physiological events. for example physical exercise.

It has been shown, that physical exercise and training profoundly influence the endocrine system (57). The hormonal changes resulting from exercise and training may be uselul as treatment for existing diseases (10), but can be harmful as well.

For example, in young women it has been demonstrated (Bonen and Keizer, 1984. Loucks and Horvath, 1985 for recent reviews), that endurance training is related to a considerably higher incidence of menstrual cycle disturbances, including secondary amenornea. In the latter case this may lead to hypo estrogenaemia and consequently to diminished bone density and osteoporosis $(60,61)$.

On the other hand, plysical exercise is possibly a valuable lool for studying the effects of stress on the hypothalamic-pituitany axis. 


\subsection{Exercise and training induced changes in plasma hormone concentrations.}

The hormonal changes induced by exercise depend on the duration and intensity of the delivered effort. It is known that acute exercise may be responsible for the immediate increase in secretion (59) and/or concentration of several hormones (58). Chronic exercise modulates the pattern of basal hormone secretion as well as the normal response to acute exercise $(9,58,63)$.

It is difficult to compare the results of various investigations, since the response of the endocrine system is dependent on:

- the exercise intensity, especially the relative (\% $\mathrm{VO}_{2}$ max) intensity. It has been shown

(64) that the intensity has to exceed $70 \%$ VOmax in order to increase the circulating levels of sex hormones, whereas an intensity of at least $80 \% \mathrm{VO}_{2}$ max is required to increase the plasma levels of prolactin and adrenocorticotropic hormone.

- the duration of the exercise bout. It seems that a minimum of 15 min of continuous exercise is required to change the circulating levels of plasma hormones $(64,69,70)$.

- the frequency of the training. In the physiology of training it is well accepted, that an optimal physical performance is only achieved after an adequate recovery (10). This means, that in athletes, the duration of the recovery phase must be taken into account in order to obtain reproducible results. This is clearly indicated by a recent study of our group in which it appears that, if training occurs too often, or in other words recovery after exercise remains incomplete, the endocrine system deteriorates (71).

Hence, it is necessary to define unambiguously the intensity and the duration of the exercise in order to compare the results of the studies dealing with this subject matter. Up to now, there has been little or no standardization in experimental design. The provided exercise is being defined as $x x$ minutes of vigorous exercise (65), exercise to a subjective maximum (66) or a certain percentage of the maximum pre-determined work capacity or maximal oxygen uptake ( $\mathrm{VO}_{2}$ max) of an individual $(64,67)$.

Generally, the maximum work capacity may increase as direct consequence of chronic exercise (training). Logically, hormonal responses to exercise may change during training as well. In consequence, it is necessary to standardize the nomenclature of the exercise intensity by expressing it as relative increase in the VOamax. Another important fact influencing the hormonal response is the duration of the exercise. It is easily understandable that differences will be present between the hormonal responses to short time and to long time exercise. It is well accepted, but poorly investigated, that the hypothalamic-pituitary axis responds to exercise and training as well. Although all the pituitary hormones are influenced, we concentrate on thyrotrophin and the gonadotrophins.

TSH and thyroid hormones:

The concentrations of the thyroid hormones $T_{3}$ and $T_{4}$ and the concentration of the free thyroid hormones rise after intensive acute exercise. Incidentally, a fall in concentration or no response to a marathon running has been reported (58). Generally, no difference is found in the plasma TSH concentration, but it must be realized that the reports have been 
made in a period that sensitive methods of TSH estimation were not avallable in a routine laboratory. Theretore, it is possible that slight changes in TSH have not been recognized. In chapter 3 of this thesis, it has been shown that slight decreases in TSH may be the result of (sight) increased thyroid hormone concentrations. Two papers mention a slight fall in plasma TSH concentiation in response to exercise $(62,68)$, but both are dated in 1984. Since sensitive methods for the estimation of TSH have been introduced in daily routine only from the midde of 1984, is is not likely that a sensitwe and specific method for TSH has been used. In consequence, a possible fall in plasma TSH concentration has to be verilted, using modern methods.

LH and FSH: in the study of the responses of the gonadotrophins to exercise, a difference must be made between the response in men and women. In females again the papers do not report congruent results: increased $(19)$, decreased $(20)$ and no responses $(21,22)$ have been described. In 1985, Cumming et al.(23), found a reduction of the pulse frequency 6 th affer acute exercise of nearly $50 \%$ in comparison to the observed frequency during exercise. In their study, the pulse amplitude did not change in a significant way.

Hence, there are little investigations dealing with the acute effects of exercise on $\mathrm{LH}$ and/or FSH pulsatility in females.

In physiology it is well accepted that an optimal physical performance is only achieved after an adequate recovery $(63,64)$. This means that in athletes, the duration of the recovery phase must be taken into account, in order to obtain reproducible results. This is clearly indicated by a recent study of our group, in which we found (14), thal after contests of 15,25 and $42 \mathrm{~km}$ gradually increasing periods of rest are needed to re-establish normal metabolism of testosterone.

\subsection{Pulse Detection.}

Mast of the pituitary hormones are supposed to be secreted episodically. The plasma concentrations are not subject to gradual smoothed changes. On the contrary, sharp increases followed by more prolonged decreases can be demonstrated Such changes reflect an episodic pituitary paltern in the hormone secretion rate. Mixing of the pituitary venous output with the systemic circulation and peripheral clearance, however, smoothes the efflusion of the hormone in the peripheral blood vessels. Due to the accompanying reduction of the differences between baseline and topvalues, the recognition of this pulsatle (or episadic) release of the hormone is difficult. Since it is believed that the episodic release of the pituitary hormones is the result of episodic stimulation by the hypothalamus, the study of the pulsatility of the pitutitary hormone concentration offers the possibility to assess hypothalamic activity.

The pulsatile behaviour of the harmone concentrations, however, must be determined in mathematical terms: pulse frequency and pulse amplitude. Furthermore, it imust be realized that due to the large smoothing effect of the mixing of the pituitary efflusion with the systemic circulation, there is a bad signal to noise ratio. Obviously, visual methods of pulse detection do not satisfy. For this reason a number of computer programs has been developed to quantify frequency and amplitude of pulses. 
Before a 'pulse' can be considered to be a pulse, some conditions must be satisfied. Assumptions about the peak form, peak height and peak frequency may be helpful in defining the algorithm of the computer program (24):

- the peak should be significantly different from the background level;

- the peaks should last for more than one measuring point;

- the contours of the peak should be smooth;

- the peaks occur at more or less regular intervals;

- the intervals between peaks should be essentially free of activity;

- the peaks should show sharp rises and should tail exponentially;

- there should be some correlation between the occurrence of peaks and the occurrences of clinical signs showing the response of the target-organ.

The use of computer algorithms permits consistency in the results. The peaks so detected satisfy unambiguously the conditions of the program and repeated analysis of the same data set will give exactly the same results. Visual methods will greatly depend on the subjectivity of the explorer. Furthermore, the scale of the plots will influence the sensitivity of peak detection. Therefore, visual methods lack in reproducibility. However, they will still be necessary in order to avoid grossly over- or under-estimation of the number of peaks. Such false positive or false negative findings occur since no algorithm will incorporate all the normal physiologic control mechanism of peaking and secondly, not all the criteria that have been incorporated, are based on truly proven physiology.

An important advantage of the use of computer algorithms is the possibility lo incorporate the analytical reproducibility avoiding false positive recognition of peaks.

\subsubsection{Description of the most important programs of pulse detection.}

The algorithm by Santen and Barden (25) was one of the first methods of objective peak detection. The method can be described as a threshold technique in which a peak is defined as a rise of at least three times the intra-assay standard deviation above the previous nadir. Only the increase is considered, but this rise must be uniform since meantime dips may hide peaks.

This widely used and adapted method has been refined by Baird (26) and Van Cauter (27). In the method of Baird the rise of the peak must exceed the baseline for at least 4 assay s.d.'s and the width must be at least two consecutive points. The extension made by Van Cauter, consists of the necessity of similarity between the increase and the decrease of a peak of at least 3 times the intra-assay s.d.

The peak detector program of Clifton and Steiner (28) is based on the interference of regular peaks with random noise. Peaks are supposed to have essentially the same form at regular intervals and uniform amplitude.

Pulsar is another important computer algorithm for the detection of peaks and has been extensively used in our study. The program has been developed by Merriam and Wachter (29). A smoothed baseline is constructed using the Lowess algorithm of Cleveland. A baseline value is calculated for each point using a fixed number of observations (window of observation). Depending on their distances to the supposed baseline, a weight is attributed to each point. Next , by weighted linear regression of the hormone 
concentrations versus time, an estimate is calculated for the baseline. This is repeated for each point in the time series. Iteration is continued by the assignment of new weights to each individual point until the influence of outliers is reduced to a minimum. This smoothing process is a kind of moving average digital filter. The width of the observation window is kept constant for all points which gives a characteristic time constant that may have a value up to 12 hours. The baseline typically can follow circadian variations but is insensitive to ultradian rhythms. The calculated baseline values for each point are subtracted from the original data to produce a residual time series that can be analyzed for the presence of peaks. The peak analysis takes both the peak height and peak width into account. A number of five cutoff criteria $G(1)-G(5)$ is used, defined in such a way, that narrow peaks must be higher than broader peaks belore they can be recognized as peaks. Correct values for these constants can be determined both by empirical methods and by theoretical approach.

Another pulse detection program that has been used, was developed by J.D. Veldhuis and coworkers $(30,8)$. In contrast to the method used in the Pulsar program, which is essentialy based on mathematics, this method is based on the normal physiology of secretion and metabolic clearance of the studied hormone. The multi parameter deconvolution will allow the estimation of amplitudes, locations and half-durations of all significant underlying secretory impulses, that give rise to the increase of plasma hormone concentrations. In figure 4.1 the concept of the convolution integral is schematically represented.

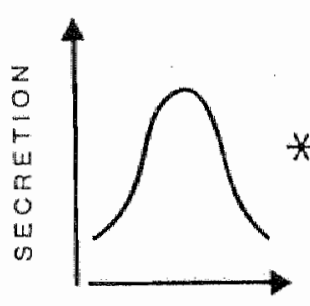

THE

$S(z)$

secretion (cumulation) irnoulse

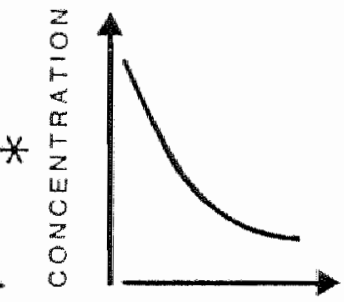

TIME

* $\quad E(t-z)$ elimination (claarance) luntion

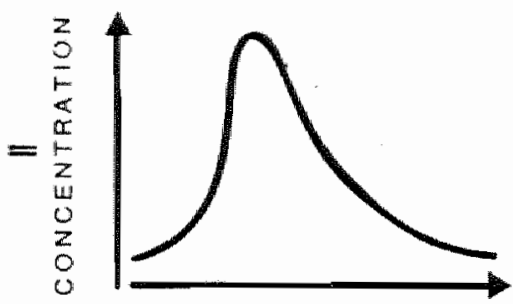

TAME

$=\quad \int_{0}^{t} S(z) E(t-z) \cdot d z$

- convalution inlegral

Figure 4.1: Convolution Integral as a superposition of a secretion and an elimination component.

The plasma hormone concentrations over a defined period are assumed to reflect the combined influence of a secretion function acted upon by mono- or bi- exponential clearance kinetics. The secretion function can have a Gaussian, Poisson or other distribution. The elimination of the hormone can be expressed depending on the available data, as a mono-, bi- or multi- exponential function. The method has proven to be very reliable, since calculated 'reconvolution fit' (a curve that is created using the best parameter estimation procedures) corresponds very closely to the plasma hormone concentrations in the time series. 


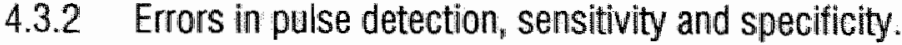

Statistical methods play a dominant role in peak detection. In time series of hormone concentrations, generally, there is a bad signal to noise ratio. Advanced algorithms are needed to recognize peaks that are aimost drowned in the background signal. Therefore, the investigation of possible errors forms an integral part of peak detection studies

\section{Urban and coauthors (8) report three types of errors:}

- false positive errors: noise is incorrectly classified as pulses, resulting in decreased specificity. This kind of error may result from sample handling (withdrawal, processing. freezing, thawing, assay) and data handling (pulse detection programs). Several methods are available to verify for the possibility that a particular procedure is subject to the detection of false positive errors;

- false negative errors: true signals are not recognized as peaks, resulting in decreased sensitivity. To determine the extent of false negative errors, exact knowledge of the true pulse frequency is necessary. However, this frequency can only be determined by indirect methods like computer simulations, concordance with different methods of estimation of hormone secretion (bioassays), increase of the sample frequency, etc: - errors due the signal being mixed with noise: in endocrinology, the true signal always will be mixed with noise. This noise can be described as noise due to the immunoassay and noise due to the sampling handling and processing before the performance of the assay. The noise from the immunoassay can be estimated using repetitive analysis of the same sample. It will show a Gaussian distribution, while the magnitude of the imprecision at a given hormone concentration can be derived from precision profiles of the assay. The noise from sampling handling and processing may be studied by the measurement in the same samples of a highly stable constituent by a method with a very low variation, e.g. total protein. Since the variation of the total protein assay is less than $1 \%$, total variation of this constituent in time series is determined merely by errors in sample withdrawal and subsequent processing. Next, the observed hormone concentration is corrected for sampling handling and processing errors by multiplying with the reverse of the concentration of total protein. Exact knowledge of the noise signal and the true hormone signal provides a basis for simulation studies, necessary in order to develop algorithms for peak detection.

Ideally, methods should be preferred having neither false positive nor false negative errors. In practice, however, less false positive errors can be obtained by more stringent treshold values, but this will result in a large number of false negative errors. Improvement of the overall methodology by the appropriate sampling frequency, low noise inducing processing of samples and immunoassays with little variance, together with peak detection algorithms based on pure physiology, rather than mathematics will be needed in further development of pulse analysis.

The measurements for the purposes of this thesis all have been done by immunoradiometric assays (IRMA) instead of radioimmunoassays (RIA). Instead of coefficients of variations (C.V.) of about $15-25 \%$ as usually found with RIA-methods, thus, 
the C.V.'s have been reduced to values of about 3-6\% . Furthermore, sampling trequencies of 1 sample per 4 minutes during rest and 1 per 8 minutes during exercise have been practiced. No efforts could be done to improve peak detection algorithms, but the methods that have been chosen are of proven rigidity.

\subsection{Pulsatility of Thyroid Stimulating Hormone.}

Nowadays, there is no doubt anymore about circadian variation of the plasma TSH concentration in normal subjects. There is a clear cut diurnal thythm with low plasma concentrations during the day and higher concentrations at evening and night. TSH concentrations rise during the evening and reach peak values before the onsel of sleep $(36,38)$. The nadir is reached about 12 hours later (around $11.00-12.00 \mathrm{~h} \mathrm{a.m.)}$.

For a long time, this knowledge of circadian variation was without clinical consequence, since the widely used routine methods for the estimation of TSH concentrations lacked in sensitivity and specificity. With the introduction of the new immunoradiometric assays for $\mathrm{TSH}_{1}$ it is possible to attain sensitivities in daily routine better than $0.04 \mathrm{mU} \mathrm{TSH} / \mathrm{I}$. Especially in pulsatility studies, sensitive and specific assays are necessary in order to increase the signal/noise ratio. The problem of pulsatility of $\mathrm{TSH}$, until now, received only moderate attention. Klingler and Ball (37) report the results of their studies in 4 healthy persons, 2 men and 2 women. Their sampling frequency was 1 sample every 2 min during $24 \mathrm{~h}$. The circadian rhythmicity had a mean frequency of $2 / 24 \mathrm{~h}$ with a maximum amplitude of $1.4 \mathrm{~mL} / \mathrm{l}$. The observed circhoral rhythmicity had a frequency of $10 / 24 \mathrm{~h}$ with an amplitude of $0.9 \mathrm{mU} / \mathrm{l}$. Moreover, they described a 'pulsatile' thythm with the mean frequency being $54 / 24 \mathrm{~h}$ and a maximum amplitude of $0.4 \mathrm{mU} / \mathrm{l}$. Greenspan et al. (32) sampled 6 normal subjects every $10 \mathrm{~min}$ and 10 euthyroid patients with underlying thyroid disease every 15 mirn. In normal subjects the mean frequency was $13 \pm 3$ pulses/24 $\mathrm{h}$ with a mean pulse amplitude of $0.6 \pm 0.2 \mathrm{mU} / \mathrm{l}$.

Clark et al.(33) sampled 10 healthy subjects in the evening only at 10 min intervals. Their mean periodicities were tound to be 60 to $100 \mathrm{~min}$. If conversion to pulses/24 $\mathrm{h}$ is allowed, the pulse frequency would be 14 to 24 pulses/24 $\mathrm{h}$. This would be in good agreement with the above studies. The authors do not mention values for mean pulse amplitude. Brabant et al. (34) uses various methods for peak detection but the results are similar. 5 men have been sampled every 10 minutes for 24 hours. Depending on the used algorithm (Pulsar or Cluster), they found a peak frequency of $5.8 \pm 1.3 \mathrm{resp} 6.8 \pm 1.9$ peaks/24 $\mathrm{h}$. The mean peak amplitude was $0.5 \pm 0.0 \mathrm{resp} 0.6 \pm 0.1 \mathrm{mU} / \mathrm{l}$.

Our study is described in detail in chapter 5 of this thesis. We sampled at intervals of 4 minutes during rest and 8 minutes during exercise from about $8.00 \mathrm{a} . \mathrm{m}$. to about 17.00 $\mathrm{pm}$. Time series have been collected of 15 women at day 7 of their menstrual cycle. In the samples both the TSH and $\mathrm{LH}$ concentrations have been measured. The mean pulse frequency as calculated with Pulsar is $16.1 \pm 1.42 / 24 \mathrm{~h}$ with a mean pulse amplitude of $0.69 \pm 0.45 \mathrm{mU} / \mathrm{l}$. In table 4.1 the results of the various studies have been compiled. In the comparison of results between the cited studies it must be realized, that clinical conditions and methodologies vary enormously. Nevertheless, similarity between the various studies seems to be present: pulse frequency has a range of \pm 6 to \pm 16 pulse/24 h and pulse amplitude goes from \pm 0.4 to $\pm 0.7 \mathrm{mU} / \mathrm{l}$. 
Table 4.1.: Pulsatility of Thyroid Stimulating Hormone in various studies.

$\begin{array}{llll}\text { Refierence } & \text { number } & \text { pulse frequency } & \text { pulse amplitude } \\ \text { Klingler et al.(37) } & 4 & 10 / 24 \mathrm{~h} & 0.4 \mathrm{mu} / \mathrm{ml} \\ \text { Greenspan et al.(32) } & 6 & 13.0 \pm 3.0 / 24 \mathrm{~h} & 0.6 \pm 0.2 \mathrm{mU} / \mathrm{l} \\ \text { Clark et al.(33) } & 10 & 14-24 / 24 \mathrm{~h} & \\ \text { Brabant et al.(34) } & 5 & 5.8 \pm 1.3 / 24 \mathrm{~h} & 0.5 \pm 0.0 \mathrm{mu} / \mathrm{l} \\ \text { this study } & 15 & 16.1 \pm 1.424 \mathrm{~h} & 0.7 \pm 0.5 \mathrm{mu} / \mathrm{l}\end{array}$

\subsection{Pulsatility of Luteinizing Hormone.}

The pulsatility of luteinizing hormone (LH) has been the object of much more study than that of TSH. Most probably "this is due to certain clinical abnormalities that can be supposed to be the consequence of abnormal pulsatility (menstrual irregularity, infertility, osteoporosis). Next, the pulsatility of $L H$ was better accessible than that of TSH since the physiologic changes of plasma hormone levels are larger and could be verified by bioassays. Although most papers deal with pulsatility of $\mathrm{LH}$, little information is given on the circadian rhythm of this hormone. However, since all pituitary hormones are controlled by central dopaminergic pathways (54) and furthermore, LH secretion both in rat and men is subject to dopamine inhibition $(48,51)$, there is no reason for an absence of a LH circadian thythm. Especially in children (for example in pubertas precox or pubertas tarda) the study of circadian rhythm might be sufficient to make a diagnosis. although the study of overall pulsatility will be more informative.

The results of some of the pulsatility studies are compiled in table 4.2 .

Table 4.2: Pulsatility of Luteinizing Hormone in various studies.

\begin{tabular}{|c|c|c|c|c|}
\hline Reference & phase of cycle & number & interpulse interval & pulse amplitude \\
\hline Veldhuis (43) & $\begin{array}{l}\text { early foll. } \\
\text { men }\end{array}$ & $\begin{array}{l}8 \\
8\end{array}$ & $\begin{array}{l}70 \pm 10 \mathrm{~min} / \mathrm{pulse} \\
73 \pm 6 \mathrm{~min} / \mathrm{pulse}\end{array}$ & $\begin{array}{l}3.6 \mathrm{mU} / \mathrm{ml} \\
4.1 \mathrm{mU} / \mathrm{ml}\end{array}$ \\
\hline Veidhuis (44) & $\begin{array}{l}\text { eary foll. } \\
\text { late foll. } \\
\text { luteral }\end{array}$ & $\begin{array}{l}6 \\
6 \\
6\end{array}$ & $\begin{array}{l}88 \pm 34 \text { minpulse } \\
65 \pm 18 \text { min/pulse } \\
198 \pm 28 \text { min/pulse }\end{array}$ & $\begin{array}{r}4.2 \pm 1.4 \mathrm{mu} / 1 \\
7.1 \pm 4 \mathrm{mu} / 1 \\
13.1 \pm 6 \mathrm{mu} /\end{array}$ \\
\hline Filicori (72) & $\begin{array}{l}\text { early loill, } \\
\text { late foll. } \\
\text { early luteal } \\
\text { late luteal }\end{array}$ & $\begin{array}{l}8 \\
8 \\
8 \\
8\end{array}$ & $\begin{array}{l}94 \pm 4 \text { min/pulse } \\
71 \pm 4 \text { minpulse } \\
103 \pm 8 \text { min/pulse } \\
216 \pm 39 \text { minpulse }\end{array}$ & $\begin{array}{r}6.5 \pm 0.4 \mathrm{mU} / \mathrm{l} \\
7.2 \pm 1.2 \mathrm{mU} / \mathrm{l} \\
1.4 .9 \pm 1.7 \mathrm{mU} / \mathrm{l} \\
7.6 \pm 1.1 \mathrm{mU} / \mathrm{l}\end{array}$ \\
\hline
\end{tabular}




\section{References:}

1. Moore Ede M.C.: The chcadian thing system in mammals: wo pacemakers preside over many secondary ascillators. Fed.Proc.(1983) 42:2802-2808

2. Kneger D.T. (edilor): Endocrine Phythms. New York: Raven Press, (1979).

3. Weitsman E.D: Biologic rhythms and homone secretion patterns. IN: Martini L, Ganong W.F. editers: Frontiers in Neurdendocrinology. Vol 5, New York: Raven Press, (1978):185-206

4. Moore R.Y.: Organization and lunction of a central nervous system circadian oscillator. Fed. Proc.(1983) $42.2783-2789$

5. Mathews D.R. Naylor B.A., Jones A.G., Ward G.M., Tumer R.C.: Pulsatile insulin has greater hypoglycemic effect than continuous delvery. Diabetes (1983) 32;617-621

6. Naftolin F. Yen S.S.C. Tsai C.C.: Rapid cycling of plasma gonadotropins in normal men as demonstrated by frequent sampling. Nalure (1972) 236;92

7. Costongs G.M.P.J., wanson P.C.W., Brombacher P.J.: Effects of biological and anatytical wariations on the appropriate use of 'reference intervals' in clinical chemistry. Proposal of a scheme for longitudinal assessment of laboratory values. J.Clin. Chiem.Clin.Biochem.(1984) 22;613-621

8. Uiban F.J., Evans W.S., Rogol A.D., Kaiser D.L., Johnson M.L. and Veldhuis J.D.: Contemporary Aspects Or Discrete Peak-Detection Aigorithms. The Paradigm of the Luteinizing Hormone Pulse Signal in Men. Endocrine Reviews (1988) 9;3-37

9. Bonen A., Ling W.Y. Macintyre K.P., Neill R., MoGrail J.C..Belcastro A.N. Effects of exercise on the serum concentrations of FSH, $\mathrm{LH}_{\text {, }}$ progesterone and estradiol. Eur.J.Appl.Physioll.(1979) 42;15-23

10. Hollman W., Hettinger Th.: Sportmedizin. Arbeits und Trainingsgrundiagen. Schattaner Verlag, Stuttgart (1976)

11. Drinkwater B.L. Nilson K., Chesnut C.H.: Bone mineral content of amenorrheic and eumenorheic athletes. N.Engl.J.Med.(1984) 311;277-281

12. Keizer H.A.: Hormonal reponses in women as a function of physical exercise and training. thesis Rijksuniversiteit Limburg, Maastricht (1983)

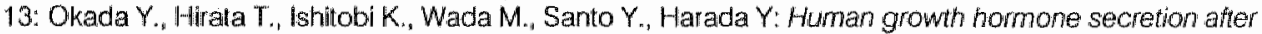
exercise and oral glucose administration in patients with short stature. J.Clin. Endocrinol.Metab.(1972) 34; $11055-1058$

14. Keizer H.A. Janssen G.M.E. Menheere P.P.C.A.: Changes in basal plasma testosterone, contisol and dehydroepiandrosterone suphate in previously untrained males and females preparing for a mavathon. Int J.Sports.Med.: In press

15. Davies C.T.M., Few J.D. Effects of exercise on adrenocontical function. J.Appl. Physiol.(1973) 35;887-889 16. Noel G.L., Suh H.K., Stone J.G., Frantz A.G.: Human prolactin and growth hormone release during surgery and other conditions of Stress. J.Clin. Endocrinal. Metab.(1972) 35;840-851

17. Cumming D.C. Brunsting L.A., Strich G. Ries A.L., Rebar R.W.: Peproductive hormone increases in response to acuke exercise in men. Medicine and Sctence in Sports and Exercise (1986) 18,369-373 18. De Meirleir K.L.,Baeyens L., L“Hermite-Baleriaux M. LLHermite M. Hollmann W.: Exercise induced prolachin velease is related to anaerobiosis. J.Clin. Endocrinol Metab.(1985) 60;1250-1252

19. Baker E.R. Mathur R.S., Klik P.F., Landgrebe S.C., Moody L.O., Williamson H.O.: Plasma gonadotropins, prolactin and steroid hormone concentrations in female runers immediatly after a longdistance run. Fentil. Steril.(1982) 38;38-41

20. Hale R.W. Kosasa T., Krieger J. Pepper S.: A marathon: The immediale effect on female runners" lutainzing homone, follicle stimukating hormone, prolactin, testosterone and contisol levels. J. Obstefrics and Gynecology (1983) $146,550-556$

21. Sutton J.R. "Coleman M.J. Casey J., Lazarus L.: Androgen responses duning physicaf exercise. Brit.Med.J. (1973) i:520-522

22. Bullen B.A. Skrinar G.S. Beitins I.Z. Carr D.B., Reppert S.M. Dotson C.O., Fend M.M., Gervino E.V. MicArthur J.W.: Endurance training effects on plasma hormonal responsiveness and sex hormone excretion. J.Appl.Physiol.(1984) 56, 1.453-1463

23. Cumming D.C., Vickovic M.M., Wall S.R., Fluker M.P., Belcastro A.N.: The effect of acute exercise on pulsatile release of hteinizing nomone in women runners. Am.d of Obstet. Gyn. (1985) 153;482.485 
24. Merriam G.P. Wachter K.W. Measurement and analysis of episodic homone secreton. IN: Rodbard D. Forti G.(editors): Computers in Endocrinology, Serono Symposia Publications, Volume 14. (1984), Paven Press, New York.

25. Santen R.ul, Bardin C.W.: Episodic luteinizhng homone secretion in men. Pulse analysis, clinical interpretation, physiologic mechanisms. J.Clin. Imest.(1973) 52;2617-2628

26. Baird D.T.: Pulsatile secretion of $L H$ and ovanan estradiof durng the follicular phase of the sheep estrous cycle. Biol.Reprod.(1978) 18;359-364

27. Cauter E. van, L'Hermite M. Copinschi G., Refetofi S, Desir D., Robyn G.: Quantitative anaysis of spontaneous variations of plasma prolactin in nomal men. Am.J.Physiol (1981) 241:E255-264

28. Clitton D.K. Steiner R.A. Cycle detection: A technique for estimating the frequency and amplitude of episodic fluctuations in blood hormone and substrate concentrations. Endocrinology (1983) 112:1057-1064

29. Merriam G.R. Wachter K.W.: Algorithms for the study of episodic homone secretion.

Am. . Physiol.(1982) 243;E310-318

30. Veldhuis J.D. "Car Ison M., Johnson M.: The pitutany gland secretes in bursts: appraising the nature of glandular secretory impulses by simultaneous multiple parameter deconwolution of plasma homone concentrations. Proc. Natn.Acad.Sci. USA (1987) 84;7686-7690

31. Hugues J.N., Reinberg A., Lagoguey M., Modigliani E.: Les yithmes biologiques de la secretion thyreotrope. Ann.Med.Irit.(1983) 134;84-94

32. Greenspan S.L., Kibanski A., Schoenleld D., Chester Ridgway E.: Pulsatile secretion of Thyrotropin in Man. J.Clin.Erudocrinol.Metab.(1986) 63;661-668

33. Clark P.M.S., Clark L.D.A., Wolder R. Raggat P.R. Wheatley T.: Pulsatile secretion of TSH in healthy subjects. Ann.Clin.Biochem.(1987) 24:470-476

34. Brabant G., Rantt $U_{\text {. Ocran K. }}$, Hesch R.D., Muhlen A. won zur: Pulsatile pattern of thyrotropin-retease in nomal men. Clin. Chim. Acta (1986) 155; 159-162

35. Evans P.J., Weeks I. Jones M.K., Woodhead J.S., Scanlon M.F.: The circadian variation of thyrotrophin in patients with primany thyroidal disease. Clin. Endocrinology (1986) 24:343-348

36: Chan V., Jones A. Liendo CH P., McNeilly A., Landon J., Besser G.M.: The relationship between circadian variations in circulating thyrotrophin, thyroid hormones and provactin. Clin. Endocrinology (1978) $9,337-349$

37. Klingler W., Ball P.: Pulsatile, circhorale und circadiane Rhythmik der TSH-Secretion beim Menschen. Nuc. Compact (1985) 16:433-440

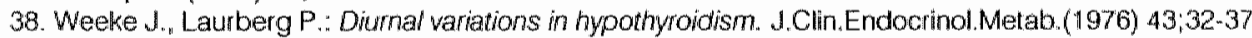
39. Barreca T., Franceschini R. Messina V., Bottaro L., Roland E.: 24-hour thyroid stimulating hormone secretory pattern in elderly men. Gerontology (1985) 31;119-123

40. Brabant G., Brabant A., Ranft U., Ocran K., Kohrle J., Hesch R.D., Muhlen A, von zur: Circadien and pulsatile thyrotropin secretion in euthyroid man under the intluence of thyroid hormone and glucoconticoid administration. لl.Clin.Endocrinol.Metab.(1987) 65;83-88

41: Murdoch A.P., Diggle P.J., Dunlop W. Kendall- Taylor P.: Determination of the frequency af pulsatile lutenizing hormone secretion by time series analysis. Clin. Endocinology (1985) 22;341-346

42: Perrild $\mathrm{H}_{\text {. }}$ Moller A. Pederson H., Siersbaek-Nielsen K.: Undetectable thyrotropin levels using immunoradiometric assay in I-dopa treated patients. J. Neurol.(1988) 235;255

43: Veldhuis J.D. Evans W.S., Johnson M.L. Wills R., Rogol A.D.: Physiological properties of the wuteinizing Hormone pulse signal: Impact of intensive and extended venous sampling paradigms on its characterization in healthy men and women. J.Clin. Endocrinol Metab.(1986) 62,881-891

44: Veldhuis J.D. Beitins I.Z., Johnson M.L., Serabian M.A., Dulau M.L.: Biologically active Luteinizing hormone is secreted in episodic pulsations that vary in relation to stage of the menstrual cycle.

J.Clin. Endocrinol.Metab.(1984) 58;1050-1058

45: Veldhuis J.D., King J.C. Urban R.J., Rogol A.D. Evans W.S., Kolp L.A., Johnson M.L.: Operating characteristics of the male hypothalamo-pitutary-gonadal axis: Pulsatile release of testosterone and follicle stimulating hormone and their temporal coupling with luteinizing hormone. J. Clin. Endocrinol. Metab.(1967) $65 ; 929-941$

46: Kazer R.R., Kessel B., Yen S.S.C.: Circulating lutenizing hormone pulse frequency in wamen with polycystic ovary syndrome. J.Clin.Endocrinol.Metab.(1987) 65:233-236 
47: Hamilton C. Heineman M.J., Mentheere P.P.C.A. Evers H. Comparison of the hormonal responses in patients with polycystic ovarian disease and normal owulating wonen following administration of a $L H A H$ agonist. IN. Roiland F. Heineman M.J. Naakigeboren N., Schoemaker J., Vemer H. Willemsen

W.N.P.(editors): Neuro-endocrinology of reproduction. Excerpta Medica, International Congress Series 751. Amsterdam (1987)

48: Steele P.A., Judd S.J.: Role of endogenous opioids in reducing the frequency of pulsatile luteinzing homone secretion induced by progesterone in normal women. Clin. Endocrinology (1986) 25:669-674 49: Chapman A.J. Wilson M.D. Obhrai M., Sawers R.S., Lynch S.S., Royston U.P. Clayton R.N.: Effect of bromocriptine on LH pulsatility in the pohycystic ovary syndrome.Clin. Endocrinology (1987) 27;571.580 50: Clitton D.K., Aksel S., Bremner W.J., Steiner R.A. Soules M.R.. Staistical evaluation of coincident prolactin and luteinizing hormone pulses duning the normal menstrual cycle. J.Clin. Endocrinol.Metab.(1988) $67 ; 832-838$

51: Orstead K.M. Hess D.L. Spies H.G.: Oplatergic inhibition of pulsatile luteinizing hormone release during the menstrual cycle of thesus macaques. Proc.Soc. Exp. Biol. Med.(1987) 184:312-319

52: Pohl C.R., Weiner R.I., Smith M.S.: Relation between luteinizing hormone and prolactin pulses in ovariectomized rats with or without dopamine inhibition. Endocrinology (1988) 123:1591-1597

53: Terasawa E., Krook C., Hei D.L., Gearing M., Schultz N.J. Davis G.A.: Norepinephrine is a possible neurotransmitter stimulating pulsatile release of luteinizing hormone veleasing hormone in the rhesus monkey. Endocrinology (1988) 123;1808-1816

54: Weiner A.L., Ganong W.F.: Pole of brain monoamines and histamine in regulation of anterior pitutary secretion. Physiol.Riev.(1978) 58;905-976

55: Llu C.M., Hedge G.A.: Factors involved in the attenuation of the TSH response to a second injection of TRH in the rat. Neuroendocrinology (1983) 36:95-105

56: Odink R.J.H.: Precocious puberty. In: Rolland R., Heineman M.J., Naaktgeboren N., Schoemaker J. Vemer H., Willemsen W.N.P.(editors): Neuro endocrinalogy of reproduction. Excerpta Medica, Internalional Congress Series 751, Amsterdam (1987)

57: Viru A.: Hormones in muscular activity. Worume l: Hormonal ensemble in exercise. CRC Press, Boca Ration (1985)

58: Bonen A. Keizer H.A. Athletic menstrual cycle irregularity: endacrine response to exercise and training Physician Sports Med.(1984) 12;78-94

59: Loucks A.N., Horvath S.M.: Athletic amenorhea : a review. Med.Sci.Sports. Exer.(1985) 17;56.72 60: Drinkwater B.L. Nilson K., Chesnut C.H.: Bone mineral content of amenorneic and eumenorrheic athlates. N. Engl.J.Med, (1984) 311;277-281

61: Lloyd T., Triantalyllow S.J., Baker E.R., Kalenak A.,Stumpi P.G., Houts P.S., Whiteside J.A.: Women athletes with menstrual cycle irregularity have increased musculoskeletal injuries.

Med.Sci.Sports.Exer.(1986) 18:374-379

62: Grossman A., Bouloux P., Price P., Drury P.L., Lam K.S.L., Turner T., Thomas J., Besser G.M., Sutton J.: The role of opioid peptides in the hormonal responses to acute exercise in man. Clin. Science (1984) $67: 483-491$

63. Boyden T.W. Pamenter A.W., Grosso D. Stantorth P., Rotkis T., Wimore J.H.: Impaired gonadotropin responses to gonadotropin releasing hormone stimulation in endurance trained women. Fertil. Sterill. (1982) 64: Keizer H.A., Kuipers H. Haan J,de, Beckers E. Habets L.: Multiple hormonal responses to physical exercise in eumenortherc trained and untrahed women. Int.J.Sports Med (1987) 8;139-150 65: Kirkeby K., Stromme S.B. Bjerkedal I. Hertzenberg L., Relsum H.E.: Effects of prolonged, strenuous exercise on lipids and thyoxine in serum. Acta Med.Scand.(1977) 202;463-467

66: Schmid P. Woll W., Pilger E., Schwaberger G., Pressenhater H.,Pristautz H., Leb G.: TSH, T3, rT3, and IT in maximal and sub-maximal physical exercise. Europ.J.Appl. Physiol.(1982) 48;31-39

67: Metvier G. Gauthier R.: The effects of acute exercise on the level of serum $T_{3}, T_{4}$ and free thyroxine incex in the aging male. J.Sportsmed.(1981) 21;371-376

68. Opstad P.K. Falch D., Oktedalen O., Fonnum F., Wergeland R.: The thyroid function in young men during prolonged exercise and the effect of energy and sleep deprivation. Clin. Endocrinology (1984) $20 ; 657-669$

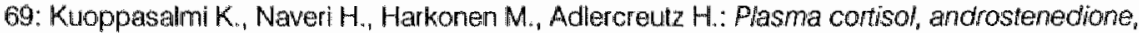
testosterone and luteinizing hormone in running exercise of different intensities. Scand.J.Clin. Invest.(1980) $40 ; 411,418$ 
70: Webo M.L., Wallace J.P., Hamill C., Hodgson J.L.Mashaly M.M.: Serum tesiosterone concenuations during two hours of moderate intensity treadmit runing in trained men and women. Endocrinol. Res. (1984) $10,27-38$

71: Kuipers H., Keizer H.A.: Overtraining in ehte athetes. Spormed (1988) 6;79.92

72. Filicori M. Santoro N. Merriam G.R. Crowley W.F.J.: Characterization of the physiological pattem of episodic gonadotropin secretion throughout the human menstrual cycle. J Clin. Endocrinol Matab. (1986) $62: 1136-1144$ 


\section{Chapter 5}

\section{Pulsatility of pituitary glycoproteins. Influence of physical exercise and two different modes of training.}

\subsection{Pulsatility of luteinizing hormone.}

\section{H.A.Keizer and P.P.C.A.Menheere}

\subsubsection{Introduction}

The prevalence of menstrual cycle irregularities in women athletes is reported to be higher than in sedentary controls (1). This observation has been attributed to several factors, among them weight loss (2), leanness (3) and age at which intensive training started (4). Although training volume has also been related to menstrual cycle irregularities, the results of the very few prospective studies $(5,6)$ carried out so far", are inconsistent. For example. Bullen et al. (7) did not observe any changes in the menstrual cycle of young eumenorheic women, who undenwent a moderately intensive endurance training program for two months. Contrastingly, the same investigators found a derangement of the menstrual cycle of eumenormeic women when training was extremely intensive and voluminous. This observation seems to be discordant with data from Shangold et al.(8), Who found an inverse relationship between training volume in the follicular phase of the menstrual cycle and the duration of the luteal phase. However, her data were collected from one subject, whereas the amount of training kilometers that the subject ran, has 10 be considered as fairly low.

Keizer et al.(9) investigated the effects of a 3-month endurance training program on plasma hormone responses during standardized bicycle ergometer work. Only minor changes in basal ovarian hormone concentrations (estradiol and progesterone) were observed. The response of both $E_{2}$ and $P$ concentrations to exercise did not change significantly with the corresponding pre-training values of follicular and luteal phases of menstrual cycle.

In marathon runners, normal menstrual cycles with progesterone levels, corresponding to normal luteal phase, were found despite their beanness and training volume (10). From the above mentioned studies, it is difficult to conclude that menstrual cycle disturbances are necessarily associated with physical exercise.

Menstrual cycle irregularities may originate at various levels.

Firstly, disturbance of the normal function of the hypothalamus may be responsible. Indeed, it has been demonstrated that the hypothalamus is influenced by psychological and cerebral conditions. In the study of Fries et al.(11), a higher incidence of secondary amenorrhea in women with a more responsible job in comparison to age-matched controls is reported. However, it remains to be proven that such influences can exent an action upon the endocrine system that is important enough to provoke menstrual irregularities. Galle et al.(12) found, in an attempt to separate the effects of running on 
physiologic and psychological factors, a difference in the mean weight and the height/weight ratio between amenorrheic and eumenorrheic runners. The psychological data showed no difference between runners and fertle controls. However, the perceived emotional distress increased with increasing mileage in all groups, indicating that other factors may be involved.

The second and probably most important level at which menstrual cycle irregularities may occur is the hypothalamic-pituitary level. To date it is generally accepted that pituitary hormones are secreted in a episodic pattern rather than being released tonically (15). The discontinuity in the production and secretion of $\mathrm{LH}$ and FSH is considered to be a prerequisite for the normal development of the follicle (16). The patterns may be described in terms of pulse frequency, pulse amplitude, pulse duration and basal hormone levels. All these characteristics of the pulsatile pattern are mainly defined at the

hypothalamic-pituitary axis. This axis is sensitive to psychological stress and perhaps also to physical stress. Boyden et al.(17) found a decreased $L H$ response to exogenous Gn-RH in adult, formerly untrained women preparing for a marathon. However, they did not observe any menstrual cycle irregularity. Several investigators reported an altered LH peak trequency (18) and/or peak amplitude (19) in eumenorrheic runners as compared to sedentary controls. Although these data suggest that regular endurance exercise might change these parameters, the immediate effects of exercise on gonadotrophin pulsatility are hardly investigated. In the only investigation dealing with this subject matter (Keizer and Bonen, (20)) no clear changes in LH and FSH peak frequency and peak amplitude were found after an acute bout ( $45 \mathrm{~min}$ ) of exhaustive exercise in untrained eumenorheic women, although there were considerable interindividual differences.

The last level at which menstrual cycle disturbances may occur is the ovary itself. In rats, it has been demonstrated that the aromatization of androgens is diminished (13). It is not known, if this will occur in women too. The increased concentration of plasma prolactin as found by Keizer et al.(9) may prevent aromatization, although the absolute levels were in the majority of cases beyond the pathological level as indicated by McNatty et al.(14). Another reason opposite to this hypothesis is the fact that lower basal levels of androgens have been found in highly trained women.

Altogether, these data suggest that regular exercise may influence the hypothalamicpituitary axis. This seems especially true, whenever a particular training is too voluminous or too intensive for a given individual. Since the effects of training are evoked by the product of training volume and -intensity and no investigations are available in which these parameters have been strictly standardized, one cannot discern between their possible effects on the hypothalamic-pituitary axis. Especially, the effects of the exercise intensity on gonadotrophin secretion pattern have not been investigated.

In order to unravel the effects of training and especially the effect of changes in relative training intensity (at a fixed training volume of $10 \mathrm{~km} / \mathrm{day}$ ) on the hypothalamicpituitary-gonadal axis and the hypothalamic-pituitary-thyroid axis at rest and during acute exercise, we investigated the effects of strictly standardized aerobic and anaerobic endurance training in 15 eumenorrheic women in the mid-follicular phase of their menstrual cycle. 


\subsubsection{Subjects, experimental design and methods.}

\section{Subjects.}

Sixteen physical education students volunteered in this study and gave their written informed consent. Their mean ( $\pm s$.d.) age, height and weight were respectively, $24.6 \pm$ $2.0 \mathrm{yr}, 172.1 \pm 5.8 \mathrm{~cm}$ and $63.9 \pm 4.9 \mathrm{~kg}$. All the volunteers met the following criteria: - a menstrual cycle length of $28 \pm 4$ days for at least 1 year prior to the study as revealed from the menses data. The normality of a control menstrual cycle was established by serial measurements of serum progesterone levels in the luteal phase in all cases P levels exceeded $15 \mathrm{nmol} / \mathrm{l}$ :

- no history of gynaecological disorders;

- normal liver and renal functions;

- no use of drugs that could interfere with menstrual cycle for at least 6 months prior to the study.

The volunteers were investigated on the $7^{\text {th }}$ day of two consecutive menstrual cycles. Prior to the start of the first experimental cycle they underwent an incremental maximal treadmill run in which at each speed stage blood from a hyperaeminized earlobe was collected for the determination of lactate (23). From this the speed at 4 mmol lactate $/ 1$ blood was determined.

\section{Experimental design.}

In the experimental period, the subjects began to train aerabically or anaerobically (5 times/week, $10 \mathrm{~km} /$ day) for the first 6 days of their menstrual cycle. The study had a cross-ower design in which the subjects were divided at random between the groups. After the first six training days, the subjects reported to the laboratory on the seventh day of two consecutive menstrual cycles. From the $8^{\text {th }}$ day to the first day of the next menstrual cycle, they trained on a low level of intensity (approximately $50 \% \mathrm{VO}$ 2max, 10 $\mathrm{km} / \mathrm{day}, 5$ times/week).

To control the intensity of the training during the first six days of the menstrual cycle, each subject ran at a predetermined lactate level as obtained from the maximall treadmill run. For this purpose blood for the determination of lactate was obtained each 10 min from a hyperaeminized earlobe. If necessary, the training intensity was tuned the next day. The aerobic training consisted of a continuous run at a speed which required about 85-90 $\%$ of the speed at which the blood lactate level reached $4 \mathrm{mmol} / \mathrm{l}$. In this case blood lactate concentration is about $1.5 \mathrm{mmol} /$ and training intensity was about $50 \% \mathrm{VO} 2 \mathrm{max}$. The anaerobic training consisted of five $800 \mathrm{~m}$ intervals at a speed of $115 \%$ of the $4 \mathrm{mmol}$ lactate threshold (this equals to about $6 \mathrm{mmol} / \|$ lactate at training intensity of $80-90 \%$ VOzmax), alternated with $800 \mathrm{~m}$ at the intensity of the aerobic runs.

The experiments on the seventh day started all between 8.00 and 10.00 am. Care was taken that the subjects were reinwestigated the second time at precisely the same time as in the lirst experiment. After insertion of a catheter in one of the antecubital veins, blood fior the determination of LH, TSH and total protein was collected every 4 min before and after 
exercise and every 8 min during exercise.

After a period of about 3 hours followed a period of about 1.5 hours during which the subject exercised. After the exercise, blood sampling continued for another 3 hours.

Exercise started on $50 \% \mathrm{VO}$ max for 8 min, thereafter the speed was increased to $65 \%$ VO2max. If there were signs that the volunteer was able to run substantially longer than 1.5 hours, the speed was gradually increased. Half of the volunteers have been stimulated three times with a combined solution of $1.25 \mu \mathrm{g} \mathrm{Gn}-\mathrm{RH}$ (Wyeth) and $1 \mu \mathrm{g} \mathrm{TRH}$ (Hoechst) at about 1 hour after the start of the experiment, about half an hour after the start of exercise and about 1 hour after cessation of exercise.

\section{Sample preparation.}

After withdrawal each sample was immediately transferred into a heparinized polyethylene tube, which was placed in an ice bath. After the experiment, the blood was centrifuged at $2000 \mathrm{~g}$ during 10 minutes at $4^{\circ} \mathrm{C}$. The plasma was collected and stored at $-20^{\circ} \mathrm{C}$ for subsequent analysis

\section{Methods.}

Total protein was determined by the biuret method, the intra-assay and inter-assay coefficients of variations were calculated to be $0.9 \%$ and $1.5 \%$, respectively. Plasma LH concentrations have been determined with the IRMA of Serono (Geneva, Switzerland as has been described in chapter 2

\subsubsection{Data analyses.}

All plasma $\mathrm{LH}$ concentrations have been corrected for changes in plasma volume due to exercise and/or dilution with saline as described by Keizer and Bonen, (20). Peak analysis of the time series of LH have been performed by visual inspection and by the PULSAR program (21), setting the $G$ values at $3.80,2.60,1.90,1.50$ and 1.20. The precision profiles were described as polynomials in order to provide correct information to the program. However, for this assay the cubic term appeared to be zero so that standard deviation was described as a linear function of hormone concentration. For LH it was found that the assay s.d. was given by: assay s.d. $=0.03$ * $[\mathrm{LH}]+0.10$. The assay sensitivity was set to $0.4 \mathrm{U} / /$ for $\mathrm{LH}$. Significance of differences in peak frequency, peak amplitude, basal concentrations and peak intervals has been tested with the Wilcoxon signed rank test, the Mann-Whitney-U test and the test of Friedman. A p level equal to or less than 0.05 was accepted as significant.

\subsubsection{Results}

The results of the time series of $\mathrm{LH}$ are graphically presented in figures 5.1.1 through 5.1.7. All figures consist of four parts, $A, B, C$ and $D$. The results of two subjects are shown beyond each other, while both the results after the aerobic (parts $A$ and $C$ ) and after the anaerobic (parts B and D) training are presented. 
Time axis is kept constant in all figures, while the hormone axis is kept constant in both the figures of one subject, but may vary from one subject to another in order to allow visual inspection in more detail.

The period of exercise is indicated by a drawn box. If applicable, the moments of stimulation with the combined solution of Gn-PH and TRH are marked. Since it is known that at normal dosages of these releasing hormones, the resulting peak in concentration of the stimulating hormone can be expected about 20 min later and that the concentration of the stimulating hormone is returned to base line about 60 min later, both these points are marked also. The peaks detected with the PULSAR program are marked by an arrow at the bottom of the figures, while a line below the arrow indicates the beginning and the end of the peaks.

Visual inspection of the figures with the time series of the LH concentration shows a possible superposition of two pulse rhythms: one with a frequency of about $1 /$ hour and another having a much higher frequency of about $1 / 20$ minutes. None of these superposed peaks have been detected by PULSAR. Since PULSAR is only based on mathematical assumptions, this is not surprising.

Unfortunately, it was not possible to apply more elaborated peak detection programs like the deconvolution model of Veldhuis et al.(26).

From various figures $(5.1: 1 a+b, 3 a+b, 3 c+d, 4 c+d, 5 b, 5 c+d, a+b, 6 c+d, 7 b, 7 c+d)$ it is obvious, that the pattern of LH pulsatility is hardly disturbed by exercise. Most of the LH time series do not even show a distinct immediate response of the hormone concentration upon exercise, while it does not make any difference if there has been an aerobic or an anaerobic training preparation.

Only some pictures (fig $5.1: 1 \mathrm{~b}, 2 \mathrm{c}, 4 \mathrm{a}, 4 \mathrm{~d}$ ) show circadian rhythm of the LH concentration. In table 5.1.1 the length of menstrual cycles of the participating subjects as function of training intensity are compiled. The menstrual cycles of the subjects were significantly longer after anaerobic training as compared to the control cycle. The length of the aerobic menstrual cycle was statistically not different from the control cycle, whereas the differences between both experimental cycles just missed significance $(p=0.08)$. In table 5.1.2 the observed pulse frequency (pulses/3 h), the pulse amplitude in [IU/l], the relative increment of the peak value above the by PULSAR established baseline and the peak areas per minute of the time series of TSH before and after exercise after 6 days of aerobic training in unstimulated subjects are compiled. Comparing the values before and after exercise, none of these pulsatility defining characteristics show significant differences. The corresponding results obtained in stimulated subjects figure in table 5.1.3. Equally, in table 5.1.4 and 5.1.5 the appropriate results after a period of six days anaerobic training are tabulated. None of the comparisons of values betore and after exercise results in a statistically significant difference. Obviously, the pulsatility pattern of $\mathrm{LH}$ does not change due to heavy exercise.

In table 5.1.6, the total area under the curve of the time series, expressed as area/min. before exercise, during and after exercise are presented for the four groups, i.e. stimulated and unstimulated both after aerobic and anaerobic training. By means of the test of Friedman, it was tested whether or not during the exercise period the mean $\mathrm{LH}$ concentration was higher than before or after exercise. In all groups the differences lacked statistical significance.

Finally, in table 5.11.7 the pulsatility characteristics in unstimulated subjects during rest 
(i.e. during the first 3 hours before exercise) after aerobic and anaerobic training are compared. Again, none of the differences was significant.

\section{Table 5.1.1:}

Length of menstrual cycles as function of training intensity

$\begin{array}{cccc}\text { Subject } & \text { Control Cycle } & \text { Aerobic Cycle } & \text { Anaerobic Cycle } \\ 1 & 21 & 25 & \\ 2 & 25 & 29 & 29 \\ 3 & 28 & 20 & 38 \\ 4 & 26 & 26 & 24 \\ 5 & 29 & 24 & 22 \\ 6 & 32 & 25 & 28 \\ 7 & 27 & 29 & 34 \\ 8 & 32 & 32 & 27 \\ 9 & 20 & 28 & 33 \\ 10 & 24 & 30 & 25 \\ 11 & 25 & 25 & 26 \\ 12 & 23 & 25 & 24 \\ 13 & 24 & 38 & 32 \\ 14 & 25 & 32 & 44 \\ 15 & 30 & 28 & 30 \\ 16 & 24 & 22 & 30 \\ & & & 25\end{array}$

mean $25.94 \quad 27.38$

29.44

$\begin{array}{llll}\text { s.d. } & 3.53 & 4.38 & 5.77\end{array}$

Wilcoxon signed rank test:

control versus aerobic: n.s.; $=0.3015$

control versus anaerobic: significant, $p=0.0362$

aerobic versus anaerobic: $n$. S., $p=0.0939$ 
Table 5.1.2.

Pulse frequency (pulses/3 hrs), pulse-amplitude [lu//], relative peak increment and peak-area per minute of time series of $\mathrm{LH}$ before and after exercise after 6 days of aerobic training in unstimulated subjects.

\begin{tabular}{|c|c|c|c|c|c|c|c|c|}
\hline & & lore exarcis & & & & After ex & & \\
\hline Subject & Freq/3 hrs & Amplitude & Rel.Iner & k Area/min & Freq/3 hrs & Amplituo & Rel.Incr. & Peak Area/min \\
\hline 1 & 3 & 1.47 & 47.6 & 6.870 & 3 & 5.28 & 167.6 & 8.429 \\
\hline & & 1.83 & 57.0 & 7.228 & & 1.58 & 51.5 & 6.020 \\
\hline & & 1.46 & 44.5 & 6.808 & & 90 & 30.1 & 3.890 \\
\hline 5 & 4 & 1.16 & 269.8 & 2.182 & 2 & 1.58 & 254.8 & 2.371 \\
\hline & & 1.01 & 229.6 & 1.600 & & 1.21 & 180.6 & 1.882 \\
\hline & & .80 & 177.8 & 1.196 & & & & \\
\hline & & 2.26 & 434.6 & 2.555 & & & & \\
\hline 6 & 1 & 1.65 & 80.5 & 6.208 & 2 & .83 & 52.2 & 5.241 \\
\hline & & & & & & 6.88 & 458.7 & 8.555 \\
\hline 7 & 1 & 1.36 & 136.0 & 3.125 & 1 & 3.41 & 294.0 & 5.794 \\
\hline 8 & 3 & .68 & 54.4. & 1.930 & 1 & 1.16 & 117.2 & 2.519 \\
\hline & & 1.28 & 104.9 & 3.755 & & & & \\
\hline & & .92 & 82.1 & 2.228 & & & & \\
\hline 16 & 3 & .88 & 36.5 & 4.201 & 3 & .70 & 35.0 & 4.168 \\
\hline & & 2.15 & 92.7 & 6.246 & & 1.84 & 96.3 & 5.621 \\
\hline & & 1.09 & 50.0 & 4.650 & & 2.14 & 115.7 & 6.306 \\
\hline mean & 2.50 & 1.33 & 126.53 & 4.052 & 2.00 & 2.29 & 154.5 & 5.066 \\
\hline s.d. & 1.22 & .48 & $110: 66$ & 2.141 & .89 & 1.94 & 127.93 & 2.193 \\
\hline Freq/3 hrs & & n.S.;p & & & & & & \\
\hline Amplitude & & A.S.; $p=$ & & & & & & \\
\hline Relincr: & & n.s.; $p=c$ & & & & & & \\
\hline Peak area & $\min :$ & n.s.; $p=0$ & 331 & & & & & \\
\hline
\end{tabular}


Table 5.1.3:

Pulse frequency (pulses/3 hrs), pulse-amplitude [UU/l], relative peak increment and peak-area per minute of time series of $\mathrm{LH}$ betore and after exercise after 6 days of aerobic training in stimulated subjects.

\section{Before exercise}

Subject Fred3 hrs Amplitude Rellincr. Peak Area/min

$\begin{array}{|lrrrr|}2 & 2 & 1.13 & 102.7 & 2.842 \\ 3 & 4 & 1.47 & 132.4 & 2.677 \\ & & 1.36 & 116.9 & 3.359 \\ & & .95 & 77.9 & 1.839 \\ & & .68 & 55.7 & 1.895 \\ 4 & 1 & 1.03 & 124.1 & 2.446 \\ 9 & 1 & 2.12 & 86.5 & 7.159 \\ & & & & \\ 10 & 1 & 1.30 & 101.6 & 3.831 \\ 11 & 3 & 1.17 & 32.3 & 6.901 \\ & & 3.79 & 106.5 & 10.034 \\ & & 1.37 & 39.4 & 7.274 \\ 12 & 2 & .38 & 21.3 & 3.128 \\ & & 1.07 & 168.2 & 3.919 \\ & & & & \\ \text { mean } & 2.00 & 1.35 & 82.62 & 4.331 \\ \text { s.d. } & 1.15 & .81 & 34.94 & 2.484\end{array}$

Fred/3 hrs Amplitude Relincr. Peak.Arearmin

$\begin{array}{lll}1.65 & 144.7 \quad 3.520\end{array}$

$\begin{array}{rrrr}2 & 1.03 & 73.1 & 3.905 \\ & .69 & 47.3 & 2.145\end{array}$

$\begin{array}{rrrr}2 & 1.03 & 73.1 & 3.905 \\ & .69 & 47.3 & 2.145\end{array}$

$.48 \quad 57.1 \quad 1.961$

$\begin{array}{lll}1.45 & 59.9 & 5.961\end{array}$

$\begin{array}{lll}1.89 & 78.1 \quad 6.518\end{array}$

$.96 \quad 70.1 \quad 3.606$

$2.39 \quad 91.2 \quad B .154$

$\begin{array}{llll}2 & 2.24 & 183.6 & 4.870\end{array}$

$1.44 \quad 121.0 \quad 3.896$

$\begin{array}{llll}1.43 & 1.42 \quad 92.6 & 4.454\end{array}$

$\begin{array}{llll}.53 & 64 & 43.83 & 1.948\end{array}$

Freq/3 hrs:

Amplitude:

n.s.j $\rho=0.104$

Belincr:

n.s.; $p=0.477$

Peak area/min:

ก...,$p=0.801$

7. 5 ." $\rho=0.527$ 
Table 5.1.4:

Pulse frequency (pulses/3 hrs), pulse-amplitude [IU/I], relative peak increment and peak-area per minute of time series of $\mathrm{LH}$ before and after exercise after 6 days of anaerobic training in unstimulated subjects.

\begin{tabular}{|c|c|c|c|c|c|c|c|c|}
\hline \multicolumn{5}{|c|}{ Before exercise } & \multicolumn{4}{|c|}{ Altar exercise } \\
\hline Subject & Fred $/ 3$ hrs & Amplitude & Rell Incr. & Pealk Area/min & Freo/3 his & Amplitude & Relincr. & Peak Area/min \\
\hline 1 & 2 & $\begin{array}{l}1.02 \\
1.15\end{array}$ & $\begin{array}{l}52.0 \\
60.8\end{array}$ & $\begin{array}{l}4.706 \\
4.289\end{array}$ & 3 & $\begin{array}{r}.87 \\
.97 \\
1.53\end{array}$ & $\begin{array}{r}50.3 \\
57.7 \\
100.7\end{array}$ & $\begin{array}{l}4.065 \\
3.150 \\
4.714\end{array}$ \\
\hline 5 & 2 & $\begin{array}{r}.37 \\
2.16\end{array}$ & $\begin{array}{r}44.0 \\
266.7\end{array}$ & $\begin{array}{l}1.775 \\
2.600\end{array}$ & 2 & $\begin{array}{l}1.68 \\
1.42\end{array}$ & $\begin{array}{l}247.1 \\
244.8\end{array}$ & $\begin{array}{l}2.875 \\
1.734\end{array}$ \\
\hline 6 & 2 & $\begin{array}{l}2.05 \\
2.02\end{array}$ & $\begin{array}{l}74.5 \\
76.8\end{array}$ & $\begin{array}{l}5.668 \\
4.891\end{array}$ & 1 & 3.24 & 144.0 & 5.881 \\
\hline 7 & 2 & $\begin{array}{r}.87 \\
2.21\end{array}$ & $\begin{array}{r}38.8 \\
130.0\end{array}$ & $\begin{array}{l}4.433 \\
5.706\end{array}$ & 0 & & & \\
\hline 8 & 3 & $\begin{array}{l}1.42 \\
1.44 \\
1.22\end{array}$ & $\begin{array}{r}99.3 \\
109.9 \\
97.6\end{array}$ & $\begin{array}{l}4.041 \\
3.879 \\
2.870\end{array}$ & 3 & $\begin{array}{r}.57 \\
.65 \\
1.00\end{array}$ & $\begin{array}{r}51.4 \\
66.3 \\
114.9\end{array}$ & $\begin{array}{l}2.336 \\
2.370 \\
2.328\end{array}$ \\
\hline 16 & 2 & $\begin{array}{r}.85 \\
2.81\end{array}$ & $\begin{array}{r}80.2 \\
246.5\end{array}$ & $\begin{array}{l}2.633 \\
4.656\end{array}$ & 3 & $\begin{array}{l}1.21 \\
1.19 \\
1.45\end{array}$ & $\begin{array}{l}62.7 \\
55.1 \\
66.5\end{array}$ & $\begin{array}{l}5.221 \\
3.348 \\
5.664\end{array}$ \\
\hline $\begin{array}{l}\text { mean } \\
\text { s.d. }\end{array}$ & $\begin{array}{r}2.17 \\
41\end{array}$ & $\begin{array}{r}1.51 \\
.69\end{array}$ & $\begin{array}{r}105.95 \\
71.92\end{array}$ & $\begin{array}{l}4.011 \\
1.247\end{array}$ & $\begin{array}{l}2.00 \\
1.26\end{array}$ & $\begin{array}{r}1.32 \\
.70\end{array}$ & $\begin{array}{c}105.1 \\
71.82\end{array}$ & $\begin{array}{l}3.641 \\
1.431\end{array}$ \\
\hline
\end{tabular}

Freq/3 hrs:

n.s.; $p=0.607$

Amplitude:

n.s.; $p=0.453$

Relincr:

n.s.; $p=0.845$

Peak area/min:

$n$.s.; $p=0.521$ 
Tabje 5.1.5:

Pulse frequency (pulses/3 hrs), pulse-amplitude [U/1], relative peak increment and peak-area per minute of time series of $\mathrm{LH}$ before and after exercise after 6 days of anaerobic training in stimulated subjects.

Belore exercise

Subject Freq/3 his Amplitude Relincr. Peak Areatroin

2

$3-1 \quad 1.59$

9

10

$$
3
$$

2.59

1.49

121.0

67.4

6.541

5.381

.72

1.03

.88

\section{$37.3 \quad 2.005$}

54.5

3.233

48.6

2.750

11

$$
2
$$

\subsection{5}

1.74

33.4

57.2

6.820

6.810

12

$1 \quad 3.25$

331.6

5.366

13

13.68

149.0

8.470

mean $\quad 1.86 \quad 1.72$

$\begin{array}{lll}\text { s.d. } & 90 & .92\end{array}$
99.00
81.77
Alter exercise

Freq/3 hrs. Amplitude Rellnor. Peak Area/min

2

$1.69 \quad 95.5$

4.395

$.67 \quad 35.6$

4.017

3

$2.13 \quad 160.2 \quad 4.993$

$\begin{array}{lll}1.42 & 97.9 & 4.313\end{array}$

$1.89 \quad 111.8 \quad 3.193$

1

$1.43 \quad 70.8 \quad 4.991$

2

$.92 \quad 52.6$

3.419

$1.37 \quad 78.3$

3.341

4

2.01

72.0

6.739

1.39

50.5

5.734

1.62

59.6

6.772

1.43

54.0

6.423

2

$1.77 \quad 100.0$

4.986

$1.68 \quad 68.9$

2.762

3.21

86.8

10.934

2.14

1.07
1.64

.58
79.6

30.94 
Table 5.1.6:

The area under the curve of time series of LH before,during and after exercise.

a: Unstimulated subjects after previous AEROBIC training.

\begin{tabular}{|c|c|c|}
\hline Subject & $\begin{array}{l}\text { Area/min before } \\
\text { exercise }\end{array}$ & $\begin{array}{l}\text { Area/min during } \\
\text { exercise }\end{array}$ \\
\hline
\end{tabular}

$\begin{array}{cccc}1 & 3.804 & 3.423 & 3.392 \\ 5 & 0.904 & 1.207 & 0.935 \\ 6 & 3.467 & 3.172 & 3.273 \\ 7 & 1.147 & 2.208 & 2.159 \\ 8 & 1.516 & 1.321 & 1.102 \\ 16 & 2.752 & 2.109 & 2.715 \\ \text { n.s.; } p>0.10 & & & \end{array}$

b: Unstimulated subjects after previous AEROBIC training.

\begin{tabular}{|c|c|c|}
\hline Subject & $\begin{array}{l}\text { Area/min before } \\
\text { exercise }\end{array}$ & $\begin{array}{l}\text { Area/min during } \\
\text { exercise }\end{array}$ \\
\hline
\end{tabular}

$\begin{array}{llll}2 & 1.548 & 1.614 & 1.582 \\ 3 & 1.513 & 1.701 & 1.575 \\ 4 & 0.913 & 0.936 & 0.879 \\ 9 & 3.068 & 2.895 & 2.947 \\ 10 & 1.542 & 1.798 & 1.578 \\ 11 & 4.491 & 3.959 & 4.104 \\ 12 & 1.657 & 1.538 & 1.880\end{array}$

n.s.; $p>0.10$

C: Stimulated subjects after previous ANAEROBIC training.

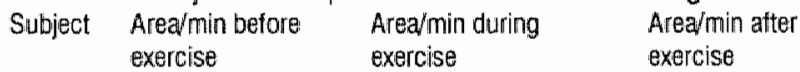

$\begin{array}{llll}1 & 2.153 & 1.920 & 1.985 \\ 5 & 1.180 & 1.256 & 1.237 \\ 6 & 2.570 & 2.432 & 2.385 \\ 7 & 2.242 & 1.923 & 0.000 \\ 8 & 1.822 & 1.556 & 1.085 \\ 16 & 1.792 & 2.128 & 2.492\end{array}$

n.S. $p>0.10$

d: Stimulated subjects after previous ANAEROBIC training.

\begin{tabular}{|c|c|c|}
\hline fect & $\begin{array}{l}\text { Area/min before } \\
\text { exercise }\end{array}$ & $\begin{array}{l}\text { Area/min during } \\
\text { exercise }\end{array}$ \\
\hline
\end{tabular}

$\begin{array}{llll}2 & 2.366 & 2.282 & 1.988 \\ 3 & 1.332 & 1.051 & 2.189 \\ 9 & 2.815 & 2.578 & 2.318 \\ 10 & 1.537 & 1.742 & 1.655 \\ 11 & 3.390 & 3.136 & 3.228 \\ 12 & 2.094 & 2.264 & 2.119 \\ 13 & 3.888 & 4.338 & 4.790\end{array}$

m.s.: $p>0.10$ 
Table 5.1.7:

Pulse frequency (pulses/3 hrs), pulse-amplitude [IU/l], relative peak increment and peak-area per minute of time series of $\mathrm{LH}$ before exercise as function of intensity of previous training in unstimulated subjects.

\section{Aller aerobic training}

Subject Frea/3 hrs Amplitude Rel.ner.

\begin{tabular}{|c|c|c|c|c|}
\hline 1 & 3 & $\begin{array}{l}1.47 \\
1.83 \\
1.45\end{array}$ & $\begin{array}{l}47.6 \\
57.0 \\
44.5\end{array}$ & $\begin{array}{l}6.870 \\
7.228 \\
6.808\end{array}$ \\
\hline 5 & 4 & $\begin{array}{r}1.16 \\
1.01 \\
.80 \\
2.26\end{array}$ & $\begin{array}{l}269.8 \\
229.6 \\
177.8 \\
434.6\end{array}$ & $\begin{array}{l}2.182 \\
1.600 \\
1.196 \\
2.555\end{array}$ \\
\hline 6 & 1 & 1.65 & 80.5 & 6.208 \\
\hline 7 & 1 & 1.36 & 136.0 & 3.125 \\
\hline 8 & 3 & $\begin{array}{r}.68 \\
1.28 \\
.92\end{array}$ & $\begin{array}{r}54.4 \\
104.9 \\
82.1\end{array}$ & $\begin{array}{l}1.930 \\
3.755 \\
2.228\end{array}$ \\
\hline 16 & 3 & $\begin{array}{r}.88 \\
2.15 \\
1.09\end{array}$ & $\begin{array}{l}36.5 \\
92.7 \\
50.0\end{array}$ & $\begin{array}{l}4.201 \\
6.246 \\
4.650\end{array}$ \\
\hline $\begin{array}{l}\text { mean } \\
\text { s.d. }\end{array}$ & $\begin{array}{l}2.50 \\
1.22\end{array}$ & $\begin{array}{r}1.33 \\
.48\end{array}$ & $\begin{array}{l}126.53 \\
110.66\end{array}$ & $\begin{array}{l}4.052 \\
2.141\end{array}$ \\
\hline
\end{tabular}

\section{After anaerobic training}

Freq/3 hrs Amplitude Rel.nncr. Area us curve

2

$1.02 \quad 52.0 \quad 4.706$

$1.15 \quad 60.8 \quad 4.289$

2

$\begin{array}{rrr}.37 & 44.0 & 1.775 \\ 2.16 & 266.7 & 2.600\end{array}$

2

$2.05 \quad 74.5 \quad 5668$

$2.02 \quad 76.8 \quad 4.891$

2

$\begin{array}{rrr}.87 & 38.8 & 4.433\end{array}$

3

$1.42 \quad 99.3 \quad 4,041$

$\begin{array}{lll}1.44 & 109.9 & 3.879\end{array}$

$\begin{array}{lll}1.22 & 97.6 & 2.870\end{array}$

2

$\begin{array}{lll}85 & 80.2 \quad 2.633\end{array}$

$2.81 \quad 246.5 \quad 4.656$

$\begin{array}{rrrr}2.17 & 1.51 & 105.9 & 4.011 \\ .41 & .69 & 71.92 & 1.217\end{array}$

freg/3 hrs:

Amplitude:

Belincr.:

Peak area/min: n. $s ; p=0.470$

n.s. $\rho=0.603$

n.s.; $p=0.846$

n.s: $p=0.758$ 

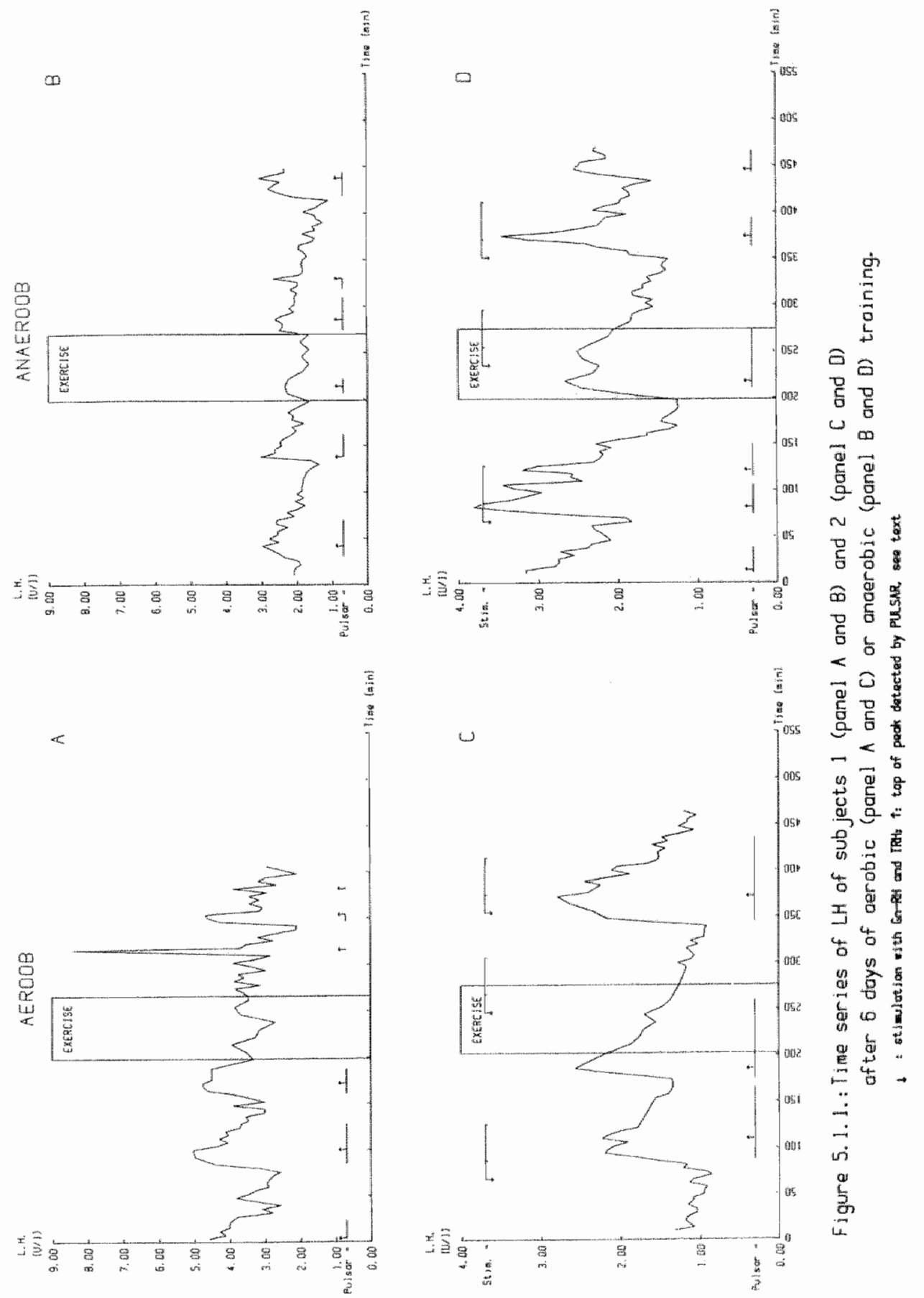


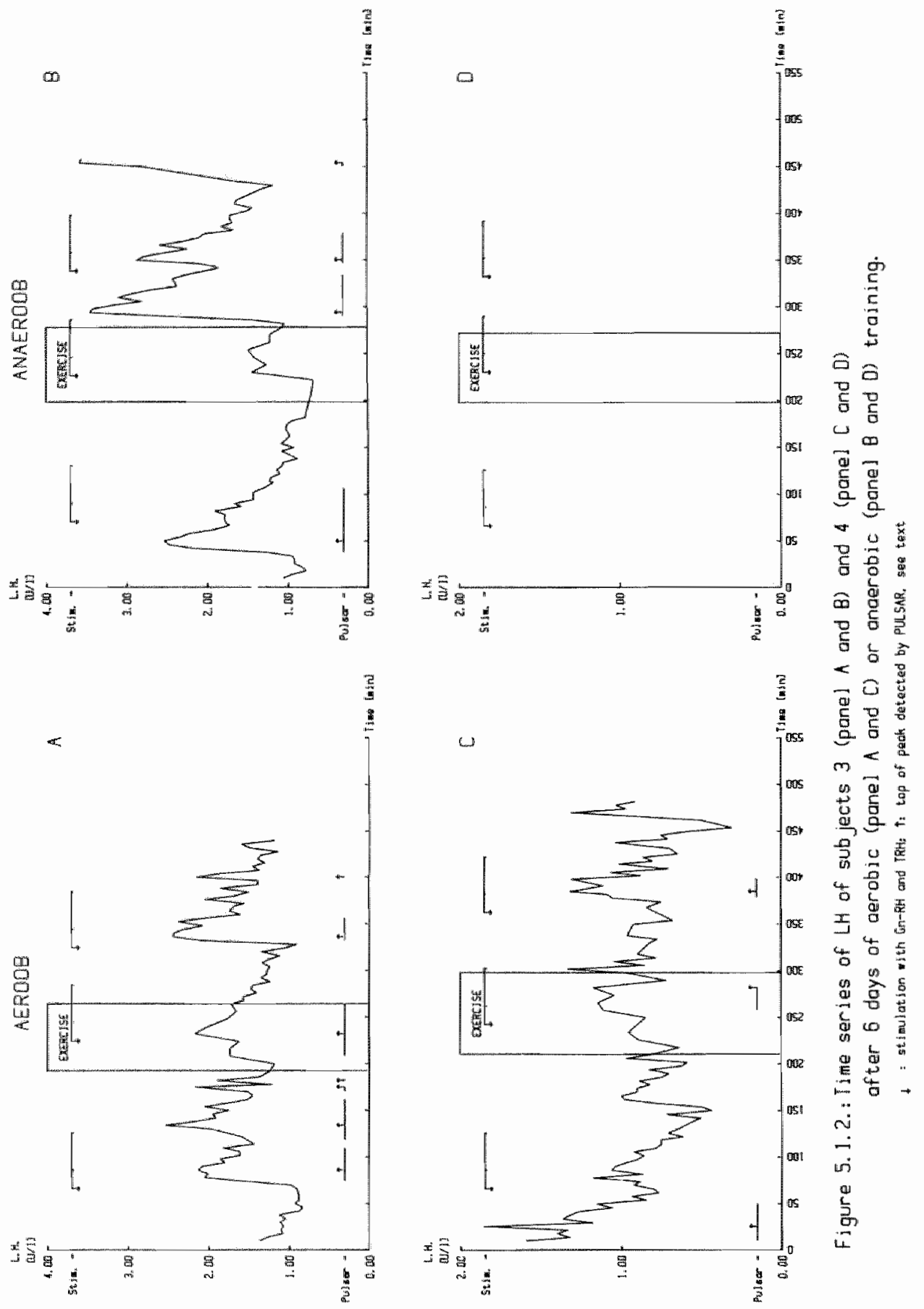



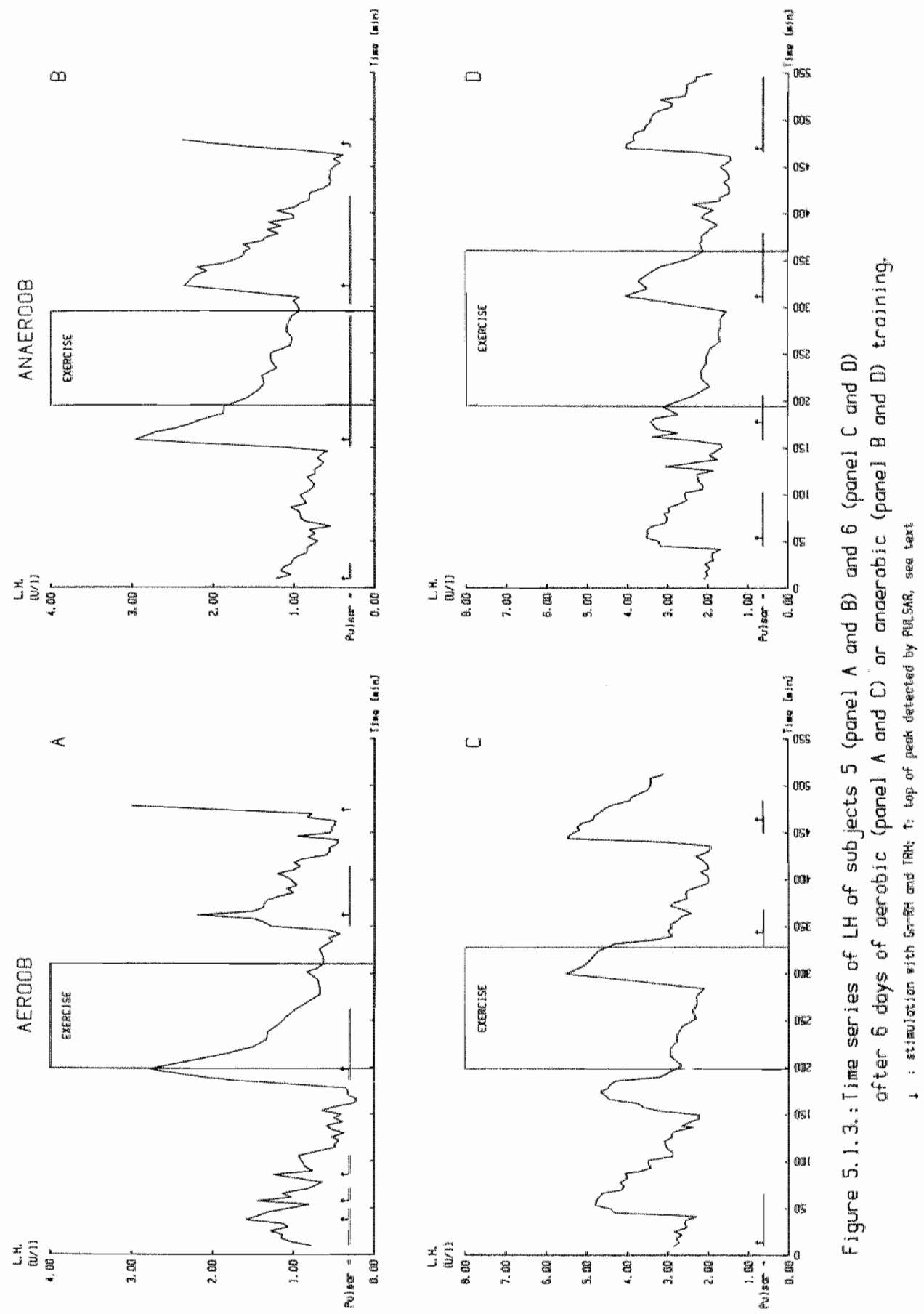

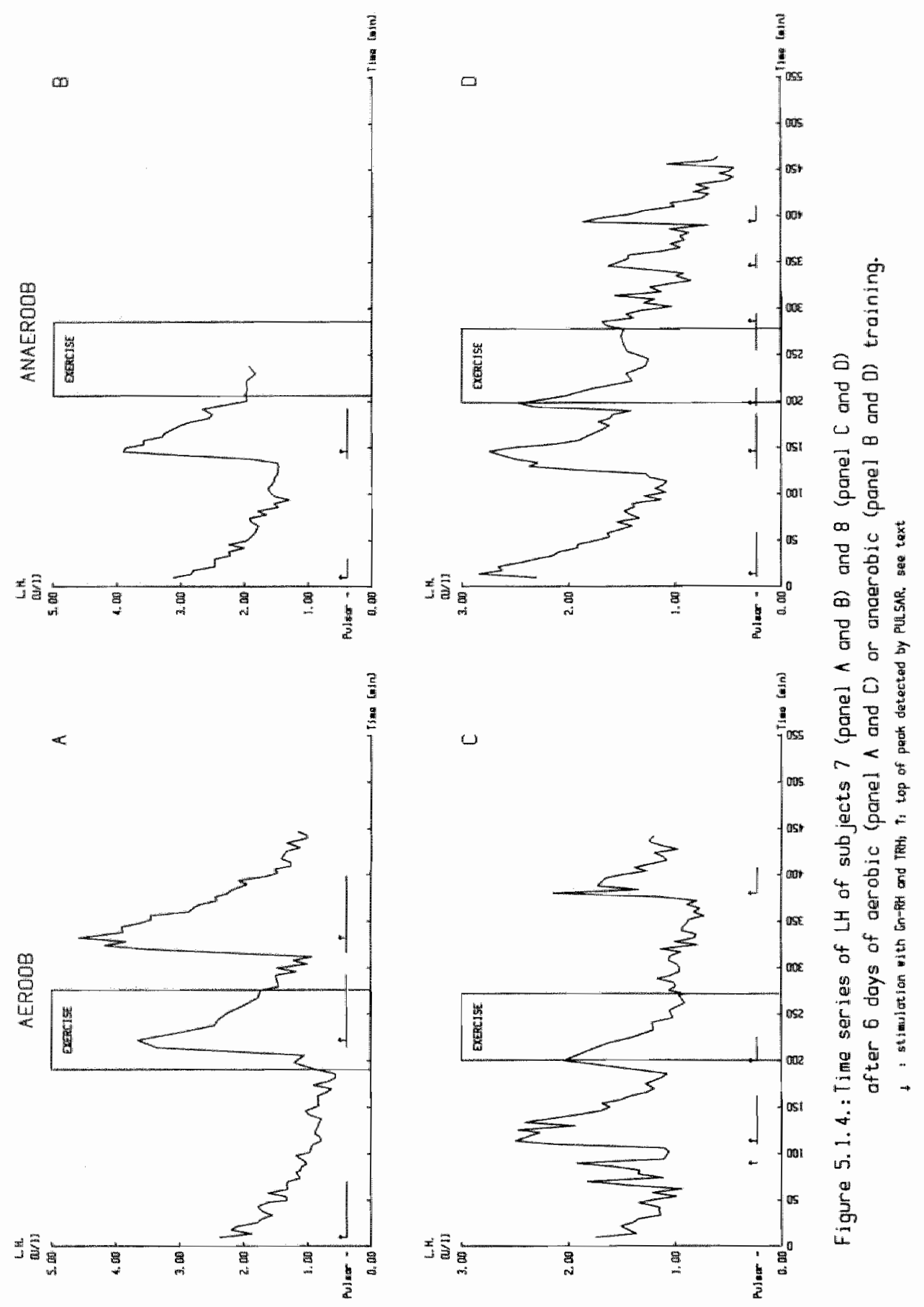


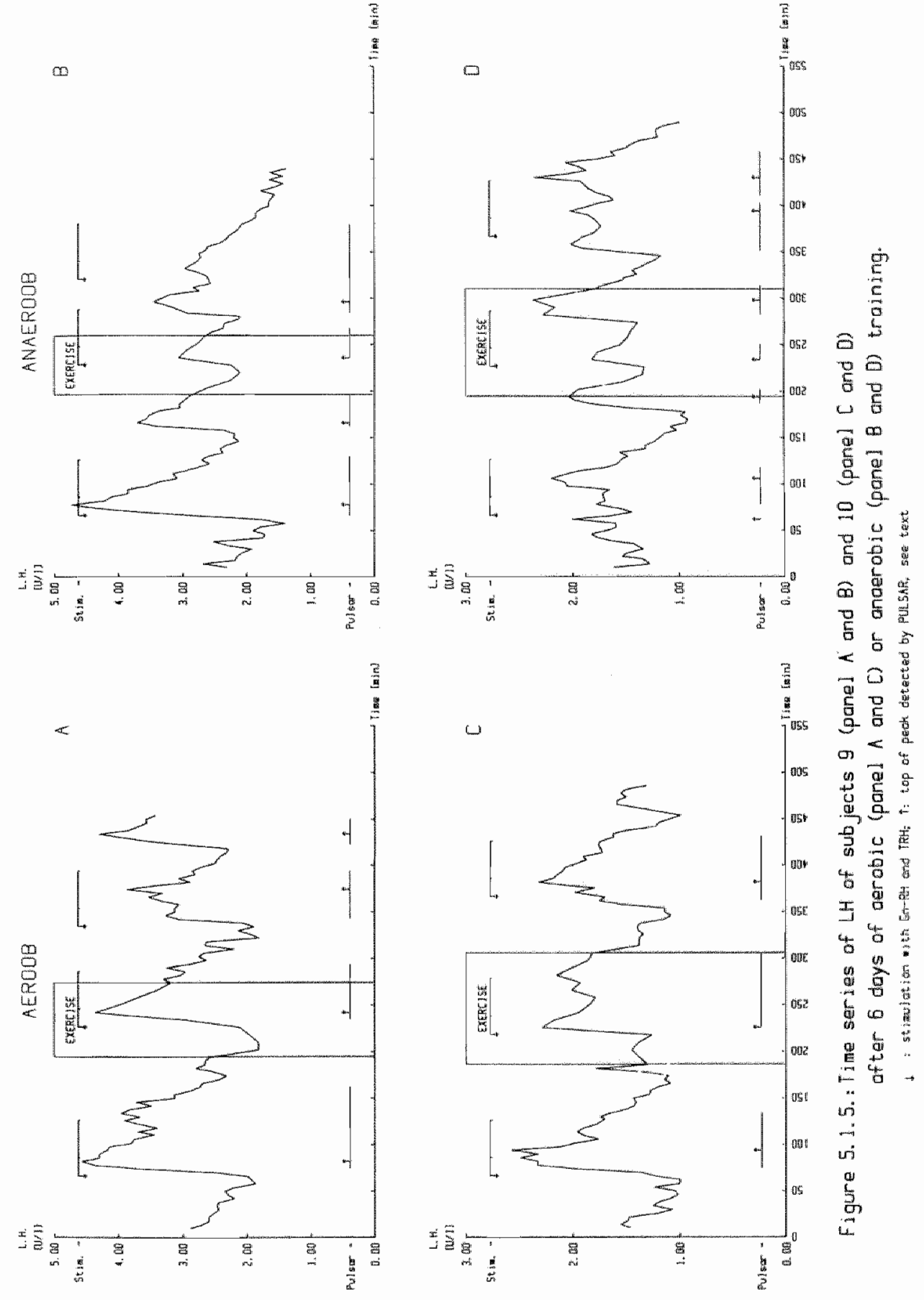



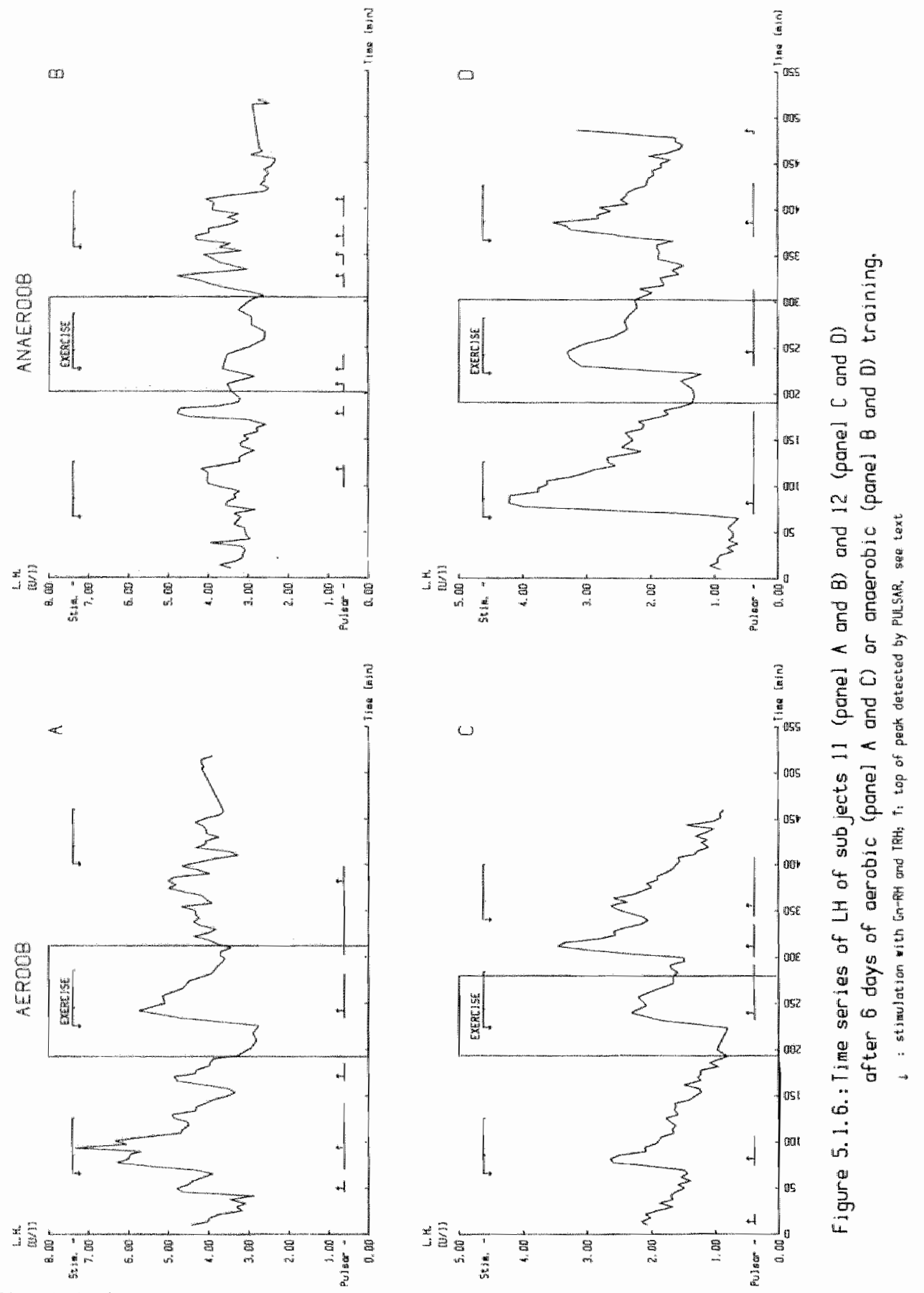

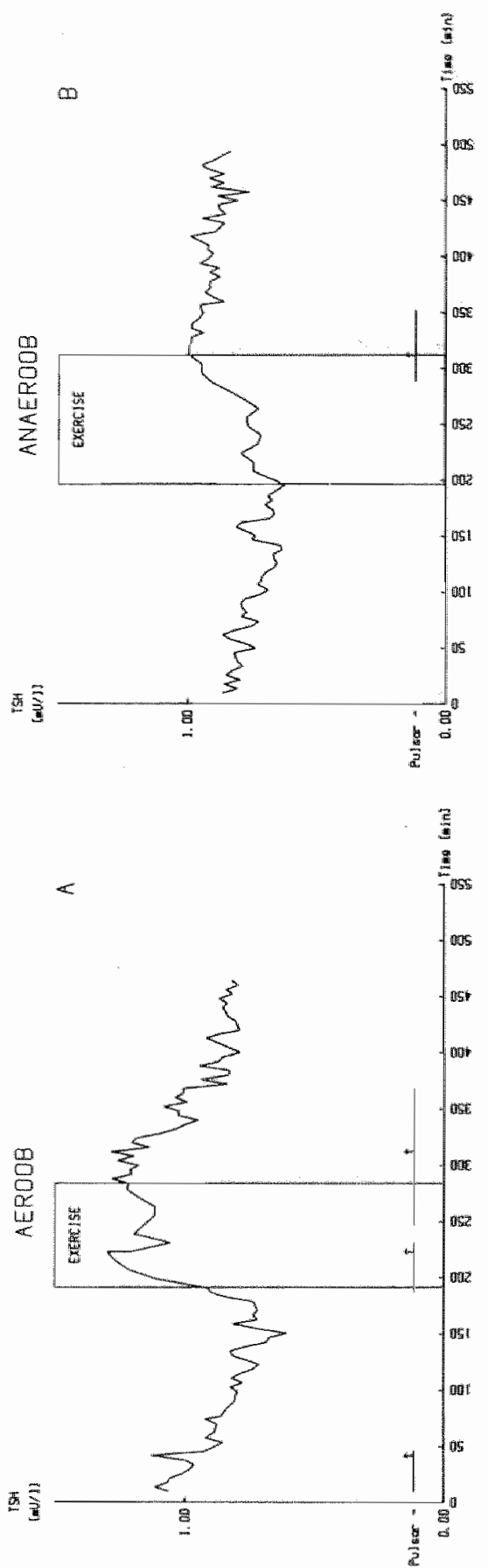


\subsubsection{Discussion}

In our study most of the subjects did not show any influence of an acute bout of exercise on LH pulsatility, neither after light nor after intensive training. In contrast, considering the whole group, a significantly lengthening of the menstrual cycle was observed. This is remarkable since the training protocol was more or less "normal'; the total volume of training ( $50 \mathrm{~km} /$ week, strictly standardized) was at a recreational level. Hence, the only variable which could change the response of the hypothalamic-pituitary axis was the exercise intensity during training, which was standardized and individualized as well. It was striking, that there were very marked interindividual differences with some quite pathological LH secretion patterns, whereas others did not show any change at all regardless the type of training. On the other hand, the menstrual cycles lengthened significantly with anaerobic training, despite the fact that the LH secretion properties did not quite reflect this. We have no explanation for these discrepancies but it might be that the exercise was not intensive enough for all subjects (i.e. a higher lactate level should have been chosen for some subjects) in order to alter the properties of the LH secretion for the whole group, or some subjects were (despite the strict standardization of the intensity) less fatigued than others.

Currently, only a very limited amount of studies on gonadotrophin pulsatility in trained versus untrained women is avallable. In general, the results of cross-sectional studies with women runners (Cumming et al. 1985; Veldhuis et al. 1985) have revealed changed LH secretion characteristics as compared to sedentary controls. For example, Cumming and co-workers (1985) showed an almost $50 \%$ lower LH pulse frequency and about $70 \%$ lower pulse amplitude in eumenorrheic runners compared to sedentary controls. The data of Veldhuis and co-workers (1985) corroborate those of the afore mentioned investigators. They found in 6 out of 9 runners with severe oligomenorrhea or secondary amenorrhea significantly lower pulse frequencies. Striking was, however, that some amenorrheic runners appeared to have quite normal LH secretion properties. This corresponds to our findings of apparently normal LH pulsatility and yet lengthening of the menstrual cycle. In summary, when the amount of training is more or less 'normal' for an individual, no clear changes could be observed in the group as a whole for the properties of pulsatility pattern of the LH secretion. We suggest training intensity is only of minor importance, if any, provided the training volume was kept constant. Unlike the unchanged LH secretion characteristics, the menstrual cycle was lengthened as a function of exercise intensity. 


\title{
5.2 Pulsatility of thyroid stimulating hormone.
}

\author{
P.P.C.A. Menheere and H.A. Keizer
}

\subsubsection{Introduction}

Physical exercise represents a complex stress situation necessitating multiple metabolic adaptations. Within the endocrine system the hypothalamic-pituitary-axis is involved in control of metabolic rate and the maintenanice of homeostasis (23). This axis is known to be a two step negative feedback mechanism (this thesis, chapter 1). It is easilly influenced at all levels by numerous external signals, like hormonal status, altered dietary state, psychological stress, etc. Exercise and training have proven to be some of the factors that may modulate the release and secretion of most of the hormones, but often the exact mechanisms behind it are unknown.

Only a limited number of investigators studied the effect of exercise and training on the hypothalamic-pituitary-thyroid axis. Recently, Boyden and Pamenter (24) published a review article about exercise and the thyroid.

Most papers agree on an increase of plasma thyroxine concentration in response to acule exercise $(25,26)$ in untrained persons. However, in trained subjects the results of the studies of the responses of thyroid hormone concentrations on acute exercise are conflicting. In two studies, increased plasma thyroxine concentrations have been reported $(27,28)$, but Theilade et al. (29) reported no significant changes of plasma thyroid hormone concentrations in a group of nine men after a $37 \mathrm{~km}$ march. Furthermore, they found no significant differences in plasma TSH concentrations. The plasma TSH concentrations have been reported to increase $(30,31)$ or to decrease $(32,33)$ in response to acute exercise. However, all these studies have been done before the era of sensitive TSH concentrations. For this reason, some scepticism with regard to the conclusions is allowed, since results might have been influenced by aspecific reactions and by the low sensitivity of the assays.

Comparison of the results of studies of the effects of chronic exercise (training) on the: thyroid hormones and TSH is very difficult, since training protocols, subject selections and methodology differ extensively. However, most studies agree on significant increased metabolic clearance rates of thyroxine, both in animals and humans $(34,35,36,37)$. De Nayer et al. (37) found increased TSH concentrations in elght athetes after four months of physical training, but they already questioned the significance of this observation because of the sensitivity of the assay.

Boyden and Pamenter (24) investigated the effects of endurance running on the thyroid axis of 17 women. During a training program with increasing intensity (from 01050 miles/week) they observed an increasing basal TSH concentration (untrained: $2.8 \pm 0.3$; at 30 miles/ week: $3.2 \pm 0.4$ and at 50 miles/week: $5.9 \pm 0.5[\mathrm{mU} / \mathrm{ll}])$. The responses of the subjects to TRH at the same training states, expressed as the area under the TSH curve above baseline TSH for 120 min after injection of $500 \mu \mathrm{g} \mathrm{TRH}$, were normal in the untrained subjects, exaggerated when training reached the level of 30 miles/week and diminished at the level of 50 miles/week. During the transient period of biochemical 
hypothyroidism, the plasma $T_{3}$ levels appeared to be significantly lower compared to both the period before training started and the end of the training program. Plasma $T_{4}$ levels behaved in the same manner.

In the literature only very limited information is available on the pulsatility of TSH and changes in pulsatility as function of different conditions. Klingler and Ball measured serum TSH with a sensitive method at 2 minutes intervals during 24 hours in 4 healthy volunteers (two men and two women, one in the peri-ovulatory period of normal menstrual cycle and one taking oral contraceptives) (38). They found three distinct patterns of TSH-rhythmicity with regard to peak frequency and peak amplitude: a circadian rhythmicity (mean frequency $2 / 24 \mathrm{~h}$; max amplitude $1.4 \mathrm{mU} / \mathrm{l}$ ), a circhoral rhythmicity (mean frequency $10 / 24$ $h_{\text {; }}$ max amplitude $0.9 \mathrm{mU} / \mathrm{l}$ ) and a 'pulsatile' rhythmicity (mean frequency 54/24h; max amplitude $0.4 \mathrm{mU} / \mathrm{i}$ ). They did not study possible influences of training status or acute exercise. Brabant et al (39) reported the existence of a pulsatile secretion pattern of TSH after investigation of the 24 hours release profile of the hormone in 7 healthy men. Blood was sampled every 10 minutes and TSH was assayed with the same sensitive assay. The time series of the hormone revealed at visual and computer assisted inspection an episodic release pattern with a low amplitude of pulses. The frequency varied depending on methodology from 7.7 peaks/24 $\mathrm{h}$ to 10.4 peaks/24 $\mathrm{h}$. Greenspan et al (40) concluded in a study of 6 normal subjects and 10 euthyroid patients that the elevations in serum TSH concentrations correlate with an increase in pulse amplitude and a decrease in pulse frequency. The nocturnal rise in TSH is associated with increases in both peak amplitude and frequency. Plasma thyroid hormone concentrations are not closely associated with pulsatile TSH secretion. Clark et al.(41) report peak frequencies of 14.4 to $24.0 / 24 \mathrm{~h}$. However, they investigated their volunteers only from $1800 \mathrm{~h}$ to $0100 \mathrm{~h}$. These peak frequencies are much higher than the above mentioned figures, but it must be realized that plasma TSH levels normally rise in the evening with an acrophase just before the onset of sleeping (38).

These experiments have been designed in order to sturdy the effects of training on the pulsatility of TSH and to study the effects of acute exercise on the episodic release of this hormone by the pituitary.

\subsubsection{Subjects, Experimental design and methods.}

This study has been performed in the same time as the study that has been described in the first part of this chapter. Therefore, the properties of the volunteers that participated as well as the experimental design are identical to that of the $\mathrm{LH}$-study, as it has been described in $5.1,2$

\section{Methods.}

Plasma TSH concentrations have been determined with the sucrosep ${ }^{\text {th }}$ - TSH IRMA of Boots-Celltech (Slough, U.K.) as has been described in chapter 2.

Total protein was determined by the biuret method. 


\subsubsection{Data analyses}

Plasma TSH concentrations have been corrected for changes in plasma volume due to exercise and/or dilution with saline as described by Keizer and Bonen, 1984. The corrections are proportional to the changes in total protein content of the sample.

Peak analysis of the time series of TSH have been performed by visual inspection and by the PULSAR program of Merriam and Wachter 1982 , setting the $G$ values at $3.80,2.60$. $1.90,1.50$ and 1.20 . The precision profile was described as a polynomial in order to provide correct information about imprecision to the program. However, for this assay the cubic term appeared to be zero so that the standard deviation could be described as a linear function of hormone concentration. For $\mathrm{TSH}_{4}$ the standard deviation was given by: assay s.d. $=0.02 *[\mathrm{TSH}]+0.04$. The assay sensitivity was set to $0.04 \mathrm{mU} / \mathrm{l}$, the mean sensitivity of 10 assays being $0.034 \mathrm{mU} / \mathrm{l}$.

Non parametrical statistic tests have been used to test the different characteristics of the pulsatility pattern (Wilcoxon signed rank test, Mann-Whitney-U test). A p level equal to or less than 0.05 was accepted as significant.

\subsubsection{Results}

The results of the time series of TSH are graphically presented in figures 5.2.1 through 5.2.7. All figures are composed of four parts, $A, B, C$ and $D$. The results of two subjects are shown beyond each other, while both the results after the aerobic (parts $A$ and $C$ ) and after the anaerobic (parts $B$ and D) training are presented. Due to blood sampling problems, we were not able to perform the analyses of both the hormones LH and TSH in all subjects. Nevertheless, in order to lacilltate the comparison of both hormones within one subject, the code number of the subjects are indicated in the legends to the figures. The time axis is kept constant in all figures. The vertical axis is kept constant in both the figures of one subject, but may vary from one subject to another in order to allow visual inspection in more detail. The period of exercise is indicated by a drawn box. If applicable the moments of stimulation with the combined solution of Gn-RH and TRH are marked. Since it is known that at normal dosages of these releasing hormones, the resulting peak in concentration of the stimulating hormone can be expected about 20 min later and that the concentration of the stimulating hormone is returned to base line about 60 min later, both these points are marked also. The peaks detected with the PULSAR program are marked by an arrow at the bottom of the figures, while a line below the arrow indicates the beginning and the end of the peaks.

Comparing the pulsatility pattern of TSH with that of LH (chapter 5.1.4) it is obvious that the pulsatility of LH is much more pronounced than that of TSH. Nevertheless, visual inspection of the figures shows as well the possibility of a superposition of two clearly distinct pulsatility rhythms, one having a frequency of about $1 /$ hour and the second being characterized by a much higher frequency of about 3-4 peaks/hour. As in the case of LH. it was not possible to recognize these intermediate peaks by means of PULSAR, using the above mentioned values for the G parameters.

In most of the plots of the TSH time series an immediate response of the TSH concentration to exercise may be observed (figures 5.2 : $1 a+b, 2 c+d, 3 a+b, 3 c, 4 c$, 
$5 a+b, 6 a+b, 6 c+d, 7 a+b)$. In stimulated subjects, the speed of increase of the TSH concentration is accelerated by the injection of TRH.

The circadian rhythm of the TSH concentration is visible in figures $5.2: 1 a+b, 2 c+d$. $3 a+b, 3 c+d, 4 a+b, 5 a+b, 5 c+d$ and $6 c$. The differences between the first values in the morning and the last values in the evening are more pronounced as basal TSH concentrations are more elevated. The intensity of previous training has no obvious influence. In table 5.2.1 the observed pulse frequency (pulses/3 h), the pulse amplitude in $[\mathrm{mU} / \mathrm{l}]$, the relative increment of the peak value above the baseline as established by PULSAR and the peak area per minute of the time series of TSH before and after exercise after 6 days of aerobic training in unstimulated subjects are compiled. Comparing the values before and after exercise, none of these pulsatility defining characteristics show significant differences. The corresponding results obtained in stimulated subjects figure in table 5.2.2. Equally, in table 5.2.3 and 5.2.4 the appropriate results after a period of six days anaerobic training are tabulated. None of the comparisons of values before and after exercise results in a statistically significant difference. Obviously, the pulsatility pattern of TSH does not change due to heavy exercise.

In table 5.2.5, the total area under the curve of the time series, expressed as area/min, before exercise, during and atter exercise are presented for the four groups, i.e. stimulated and unstimulated both after aerobic and anaerobic training. By means of the test of Friedman, it was tested whether or not during the exercise period the mean TSH concentration was higher than before or after exercise. In the groups $b, c$ and $d$ the test statistic just missed significance ( $p=0.051, p=0.052$ and $p=0.052$ resp.).

Finally, in table 5.2.6 the pulsatility characteristics in unstimulated subjects during rest (i.e. during the first 3 hours before exercise) after aerobic and anaerobic training are compiled. None of the differences was significant. 
Table 5.2.1:

Pulse frequency (pulses/3 hrs), pulse-amplitude [mU/], relative peak increment and peak-area per minute of time series of TSH before and after exercise after 6 days of aerobic training in unstimulated subjects.

\section{Before exercise}

Subject Freq/3 hrs Amplitude Rel.Incr. Peak Area/min

\begin{tabular}{|c|c|c|c|c|}
\hline 1 & 1 & .38 & 29.7 & 1.358 \\
\hline \multirow[t]{2}{*}{6} & 2 & .33 & 31.7 & .523 \\
\hline & & .22 & 21.4 & .649 \\
\hline 7 & 1 & .23 & 11.9 & 3.162 \\
\hline \multirow[t]{3}{*}{8} & 3 & .62 & 63.3 & 1.674 \\
\hline & & .61 & 63.5 & 1.566 \\
\hline & & .26 & 36.6 & .683 \\
\hline \multirow[t]{2}{*}{15} & 2 & .79 & 38.7 & 4.417 \\
\hline & & .68 & 39.8 & 2.972 \\
\hline 16 & 1 & .31 & 37.8 & 1.858 \\
\hline mean & 1.67 & .44 & 37.44 & 1.886 \\
\hline s.d. & .82 & .21 & 16.19 & 1.268 \\
\hline
\end{tabular}

After exercise

Freq/3 his Amplitude Rel.incr. Peak Arealinin

7

$\begin{array}{rrr}.87 & 107.4 & 1.783 \\ .60 & 77.9 & 1.402 \\ .44 & 62.9 & .935 \\ .53 & 77.9 & 1.147 \\ .72 & 110.8 & 1.068 \\ .51 & 81.0 & .880 \\ .36 & 61.0 & .781\end{array}$

4

$52 \quad 53.1$

.759

$\begin{array}{ll}.33 & 33.7\end{array}$

.744

.449

.365

$.29 \quad 31.2$

3.693

7

$1.19 \quad 77.8$

$\begin{array}{rrr}.40 & 65.6 & .738 \\ .31 & 51.7 & .667 \\ .50 & 84.7 & .795 \\ .47 & 83.9 & .977 \\ .54 & 100.0 & .735 \\ .40 & 75.5 & .769 \\ .40 & 78.4 & .725\end{array}$

1

$2.27 \quad 147.4$

4.853

1

47

57.3

2.054

3.50

2.95 n.s.; $p=0.112$

n.s.; $p=0.253$

significant; $p=0.001$

n.s.; $p=0.235$

$$
\text { n.s., } p=0.235
$$

Freq/3 hrs:

Amplitude:

Relincr::

Peak area/min: 
Table 5.2.2:

Pulse frequency (pulses/3 hrs), pulse-amplitude [mU/], relative peak increment and peak-area per minute of time series of TSH before and after exercise after 6 days of aerobic training in stimulated subjects.

\begin{tabular}{|c|c|c|c|c|c|c|c|c|}
\hline \multicolumn{5}{|c|}{ Before exercise } & \multicolumn{4}{|c|}{ After exercilse } \\
\hline Stubject & Frea/3 hrs & Amplitude & Relilncer. & Peak Areaimin & Freo/3 hes & Amplitude & Rellincr. & Peak Area/min \\
\hline 2 & 1 & .40 & 690 & 1.544 & 3 & $\begin{array}{l}.35 \\
.52 \\
.31\end{array}$ & $\begin{array}{l}53.0 \\
77.6 \\
46.3\end{array}$ & $\begin{array}{l}1.505 \\
1.439 \\
1.316\end{array}$ \\
\hline 3 & 2 & $\begin{array}{l}37 \\
29\end{array}$ & $\begin{array}{l}29.4 \\
23.0\end{array}$ & $\begin{array}{l}2.076 \\
1.847\end{array}$ & 1 & 1.47 & 96.1 & 3.489 \\
\hline 4 & 4 & $\begin{array}{l}.59 \\
.58 \\
.30 \\
.52\end{array}$ & $\begin{array}{l}45.4 \\
45.7 \\
23.6 \\
41.3\end{array}$ & $\begin{array}{l}2.453 \\
1.279 \\
1.587 \\
1.068\end{array}$ & 0 & & & \\
\hline 9 & 1 & .65 & 425 & 3.507 & 3 & $\begin{array}{r}1.38 \\
.56 \\
2.78\end{array}$ & $\begin{array}{r}78.4 \\
31.5 \\
155.3\end{array}$ & $\begin{array}{l}4.040 \\
3.557 \\
2.631\end{array}$ \\
\hline 10 & 2 & $\begin{array}{r}1.55 \\
.74\end{array}$ & $\begin{array}{l}74.5 \\
38.3\end{array}$ & $\begin{array}{l}3.266 \\
2.838\end{array}$ & 1 & 3.28 & 157.7 & 2.778 \\
\hline 11 & 1 & .18 & 13.2 & 2.278 & 1 & .38 & 34.5 & 2.119 \\
\hline 12 & 1 & .95 & 41.5 & 4.740 & 1 & 1.55 & 70.1 & 5.447 \\
\hline $\begin{array}{l}\text { mean } \\
s, d\end{array}$ & 1.71 & .59 & 40.61 & 2.373 & 1.43 & 1.26 & 80.1 & 2.832 \\
\hline s.d. & 1.11 & .37 & 17.80 & 1.066 & 1.13 & 1.06 & 45.16 & 1.325 \\
\hline
\end{tabular}

Froq/3 hrs.

Amplitude:

Rielincer.

Poak area/min: n.s: $p=0.618$

$n . s_{\text {. }} p=0.208$

significant; $p=0.010$

n. $S_{2 ;} p=0.435$ 
Table 5.2.3:

Pulse frequency (pulses/3 hrs), pulse-amplitude [mU/l], relative peak increment and peak-area per minute of time series of TSH before and after exercise after 6 days of anaerobic training in unstimulated subjects.

Belore exercise Subject Freq/3 hrs Amplitude Rel.lncr. PeakArea/min

1

5

.36

34

23

46

42

$\begin{array}{rrr}.49 & 53.3 & 1.851 \\ 1.54 & 169.2 & 1.460 \\ 28 & 30.8 & 1.009 \\ .32 & 36.0 & .651\end{array}$

24.8

23.8

16.3

32.6

30.0

.833

.650

.871

.774

.495
$7 \quad 0$

B

4

.30
.38
.41
.40

29.7

37.6

41.4

44.0
After exercise

Freq/3 hrs Amplitude Rellnor. Paak Area/min

4

$\begin{array}{rrr}1.21 & 145.8 & 2.149 \\ .54 & 66.7 & 1.465 \\ .43 & 54.4 & 1.446 \\ .40 & 50.0 & 1.069\end{array}$

3

$\begin{array}{lll}.59 & 43.1 & .751\end{array}$

$\begin{array}{lll}.22 & 16.1 & .843\end{array}$

$\begin{array}{lll}.32 & 23.5 & .667\end{array}$

4

$\begin{array}{lll}.97 & 63.0 & 3.698\end{array}$

$\begin{array}{lll}.85 & 58.2 & 2.729\end{array}$

$\begin{array}{lll}.39 & 28.3 & 2.753\end{array}$

$\begin{array}{lll}.64 & 58.2 & 1.743\end{array}$

$\begin{array}{lll}.19 & 23.5 & 1.153\end{array}$

$.28 \quad 36.4 \quad .866$

$\begin{array}{lllll}15 & 1 & 64 & 55.7 & 2.730\end{array}$

$16 \quad 0$

$\begin{array}{lrrrr}\text { mean } & 2.33 & .47 & 44.65 & 1.152 \\ \text { s.d. } & 2.25 & .32 & 37.48 & .591\end{array}$
2

1.081

1.080

1.571

2.730

1

1

2.50
$1.38 \quad 116.9$

$.18 \quad 22.0$

1.651

$.57 \quad 53.7$

$.37 \quad 35.95$
1.757

976
Freq/3 hrs:

Amplitude:

Relincr:

Peak area/min

$$
\begin{aligned}
& \text { n.s.; } p=0.743 \\
& \text { n.s.; } p=0.578 \\
& \text { n.s.; } p=0.387 \\
& \text { n.s.: } p=0.070
\end{aligned}
$$


Table 5.2.4:

Pulse frequency (pulses/3 hrs), pulse-amplitude [mU/7], relative peak increment and peak-area per minute of time series of TSH before and after exercise after 6 days of anaerobic training in stimulated subjects.

Betore exercise

Subject Freq/3 hrs Amplitude Reliner. Peak Area/min

\begin{tabular}{|c|c|c|c|c|}
\hline 2 & 1 & 57 & 71.3 & 1.893 \\
\hline 3 & 1 & .80 & 54.4 & 2.811 \\
\hline 4 & 3 & $\begin{array}{l}.46 \\
.38 \\
.37\end{array}$ & $\begin{array}{l}26.0 \\
23.9 \\
23.7\end{array}$ & $\begin{array}{l}2.390 \\
1.448 \\
2.238\end{array}$ \\
\hline 9 & 2 & $\begin{array}{l}.30 \\
.96\end{array}$ & $\begin{array}{l}16.9 \\
53.9\end{array}$ & $\begin{array}{l}2.007 \\
3.000\end{array}$ \\
\hline 10 & 2 & $\begin{array}{r}1.22 \\
.47\end{array}$ & $\begin{array}{l}80.3 \\
34.3\end{array}$ & $\begin{array}{l}4.250 \\
3.256\end{array}$ \\
\hline 11 & 2 & $\begin{array}{l}.24 \\
.67\end{array}$ & $\begin{array}{l}2.2 .0 \\
73.6\end{array}$ & $\begin{array}{l}1.730 \\
0.000\end{array}$ \\
\hline 12 & 3 & $\begin{array}{r}.62 \\
1.08 \\
.42\end{array}$ & $\begin{array}{l}16.0 \\
29.8 \\
12.1\end{array}$ & $\begin{array}{l}5.285 \\
5.609 \\
2.969\end{array}$ \\
\hline $\begin{array}{l}\text { mean } \\
\text { s.d. }\end{array}$ & $\begin{array}{r}2.00 \\
.82\end{array}$ & $\begin{array}{l}.61 \\
.30\end{array}$ & $\begin{array}{l}38.45 \\
23.47\end{array}$ & $\begin{array}{l}2.778 \\
1.498\end{array}$ \\
\hline
\end{tabular}

\section{After exercise}

Freq/3 hrs Amplitude Rel.ncr. Pleak Area/min$$
1
$$

$.22 \quad 33.8$ .865

2

$\begin{array}{lll}68 & 46.6 & 2.583\end{array}$

$.83 \quad 57.2 \quad 2.764$

3

$\begin{array}{lll}1.70 & 137.1 \quad 2.587\end{array}$

$1.02 \quad 85.0 \quad 2.436$

$\begin{array}{lll}.47 & 42.0 & 1.903\end{array}$

2

$1.31 \quad 64.5 \quad 3.136$

$1.27 \quad 58.3 \quad 3.594$

$\begin{array}{llll}1 & 65 & 53.3 & 5.096\end{array}$

0

0

1.29

1.11
$.94 \quad 64.2$

$.46 \quad 30.98$
2.774

1.161

\footnotetext{
Freq/3 hrs:

Amplitude:

N.S.; $p=0.704$

Piolincr:

r. $.5 ; p=0.079$

Peak arealmin:

significant; $p=0.035$

$n, s ; p=0.883$
} 
Table 5.2.5:

The area under the curve of time series of TSH before, during and after exercise.

a: Unstimulated subjects after previous AEROBIC training.

\begin{tabular}{|c|c|c|}
\hline bject & $\begin{array}{l}\text { Area/min before } \\
\text { exercise }\end{array}$ & $\begin{array}{l}\text { Area/min during } \\
\text { exercise }\end{array}$ \\
\hline
\end{tabular}

$\begin{array}{llll}1 & 0.908 & 1.219 & 0.776 \\ 6 & 0.357 & 0.458 & 0.404 \\ 7 & 1.925 & 2.155 & 1.730 \\ 8 & 0.698 & 0.527 & 0.538 \\ 15 & 1.999 & 3.210 & 2.097 \\ 16 & 0.843 & 1.159 & 0.974\end{array}$

n.s.: $p>0.10$

b: Unstimulated subjects after previous AEROBIC training.

$\begin{array}{llll}\text { Subject } & \begin{array}{l}\text { Area/min before } \\ \text { exercise }\end{array} & \begin{array}{l}\text { Area/min during } \\ \text { exercise }\end{array} & \begin{array}{l}\text { Area/min atter } \\ \text { exercise }\end{array} \\ 2 & 0.612 & 0.674 & 0.764 \\ 3 & 1.060 & 1.344 & 1.652 \\ 4 & 1.129 & 1.678 & 1.147 \\ 9 & 1.579 & 1.989 & 2.160 \\ 10 & 1.417 & 1.846 & 1.441 \\ 11 & 1.340 & 1.305 & 0.998 \\ 12 & 2.259 & 3.635 & 2.934\end{array}$

c: Stimulated subjects after previous ANAEROBIC training.

\begin{tabular}{|c|c|c|}
\hline Subject & $\begin{array}{l}\text { Area/min before } \\
\text { exercise }\end{array}$ & $\begin{array}{l}\text { Area/min during } \\
\text { exercise }\end{array}$ \\
\hline
\end{tabular}

$\begin{array}{llll}1 & 0.861 & 1.304 & 0.959 \\ 6 & 0.592 & 0.833 & 0.565 \\ 7 & 2.016 & 2.331 & 1.587 \\ 8 & 0.859 & 0.952 & 0.609 \\ 15 & 1.282 & 2.186 & 1.623 \\ 16 & 0.743 & 0.799 & 0.916\end{array}$

n.s.: $p=0.052$

d: Stimulated subjects after previous ANAEROBIC training.

$\begin{array}{llll}\text { Subject } & \begin{array}{l}\text { Area/min before } \\ \text { exercise }\end{array} & \begin{array}{l}\text { Area/min during } \\ \text { exercise }\end{array} & \begin{array}{l}\text { Area/min after } \\ \text { exercise }\end{array} \\ 2 & 0.875 & 0.999 & 0.695 \\ 3 & 1.309 & 1.396 & 1.388 \\ 4 & 1.185 & 1.676 & 1.156 \\ 9 & 1.370 & 1.847 & 1.891 \\ 10 & 1.793 & 2.816 & 2.475 \\ 11 & 1.049 & 1.325 & 0.936 \\ 12 & 2.825 & 3.799 & 3.015 \\ \text {. } p=0.052 & & & \end{array}$


Table 5.2.6:

Pulse frequency (pulses/3 hrs), pulse-amplitude [mU/]], relative peak increment and peak-area per minute of time series of TSH before exercise as function of intensity of previous training in unstimulated subjects.

\begin{tabular}{|c|c|c|c|c|c|c|c|c|}
\hline \multicolumn{5}{|c|}{ After aerobic training } & \multicolumn{4}{|c|}{ After anaetrobic training } \\
\hline Subject & Freq/3 hrs: & Amplitude & Relincr. & Area u curve & Freq/3 hrs & Amplinude & Rellnar. & Area u curve \\
\hline$\|$ & 1 & 38 & 29.7 & 1.358 & 4 & .49 & 53.3 & 1.851 \\
\hline & & & & & & 1.54 & 169.2 & 1. 460 \\
\hline & & & & & & .28 & 30.8 & 1.009 \\
\hline & & & & & & .32 & 36.0 & .651 \\
\hline 6 & 2 & .33 & 31.7 & .523 & 5 & 36 & 24.8 & .833 \\
\hline & & .22 & 21.4 & .649 & & 34 & 23.8 & .650 \\
\hline & & & & & & 23 & 16.3 & .871 \\
\hline & & & & & & .46 & 32.6 & .774 \\
\hline & & & & & & .42 & 30.0 & .495 \\
\hline 7 & 1 & .23 & 11.9 & 3.162 & 0 & & & \\
\hline 8 & 3 & .62 & 63.3 & 1.674 & 4 & .30 & 29.7 & 1.076 \\
\hline & & .61 & 63.5 & 1.566 & & .38 & 37.6 & 1.081 \\
\hline & & .26 & 36.6 & .683 & & .41 & 41.4 & 1.080 \\
\hline & & & & & & .40 & 440 & 1.571 \\
\hline 15 & 2 & .79 & 38.7 & 4.417 & 1 & .64 & 55.7 & 2.730 \\
\hline & & .68 & 39.8 & 2.972 & & & & \\
\hline 16 & 1 & 31 & 37.8 & 1.858 & D & & & \\
\hline mean & 1.67 & .44 & 37.44 & 1.886 & 2.33 & .47 & 44.7 & 1.152 \\
\hline s.d. & .82 & .21 & 16.19 & 1.268 & 2.25 & .32 & 37.48 & .591 \\
\hline
\end{tabular}

Freq/3 $\mathrm{hrs}:$

Amplitude:

n.s.; $p=0.537$

Rolinor:

Peak aroa/min: $n . s_{\text {, }} p=0.763$

n.s. $p=0.891$

$n_{i} s_{s,} ; 0=0.175$ 

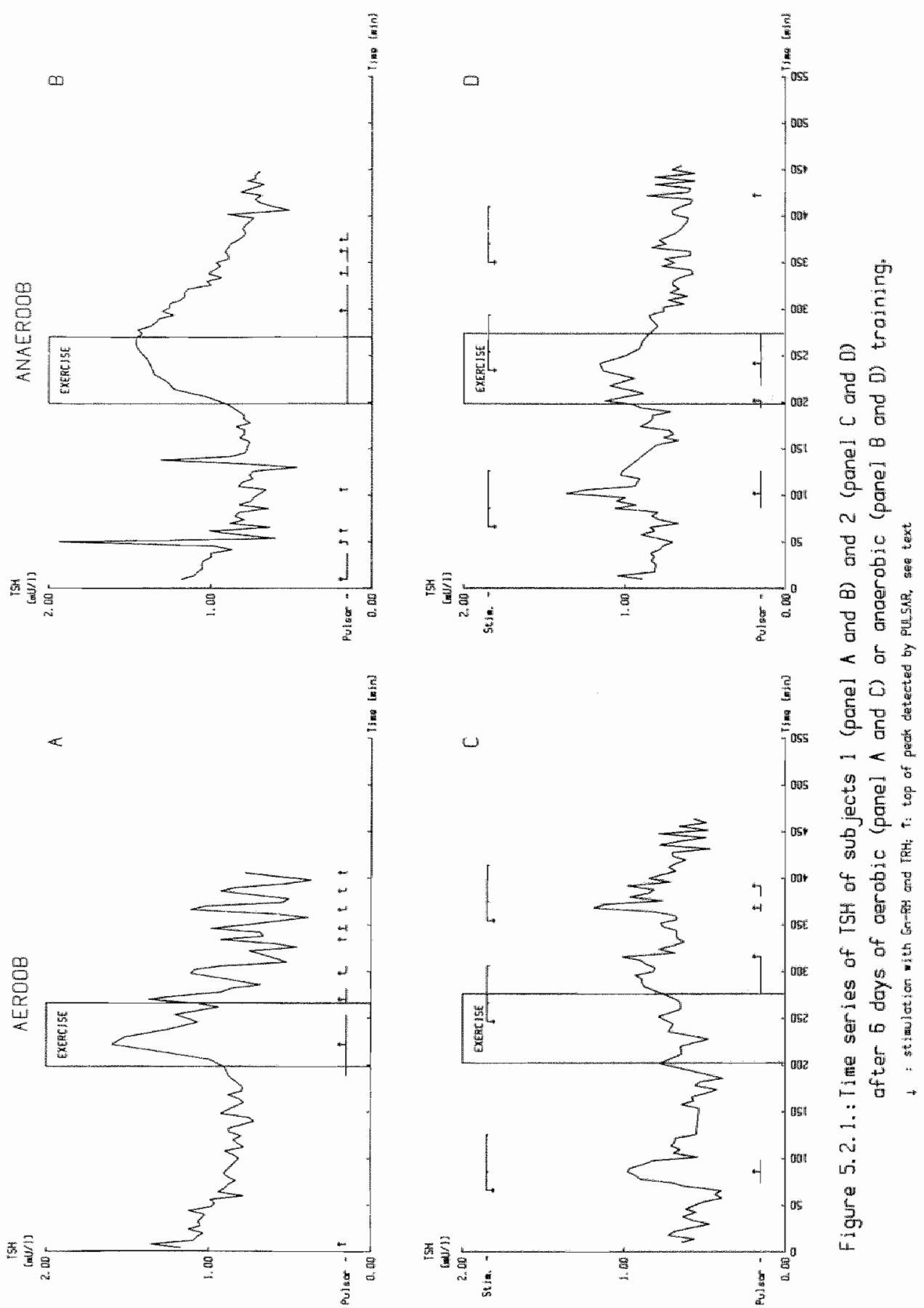

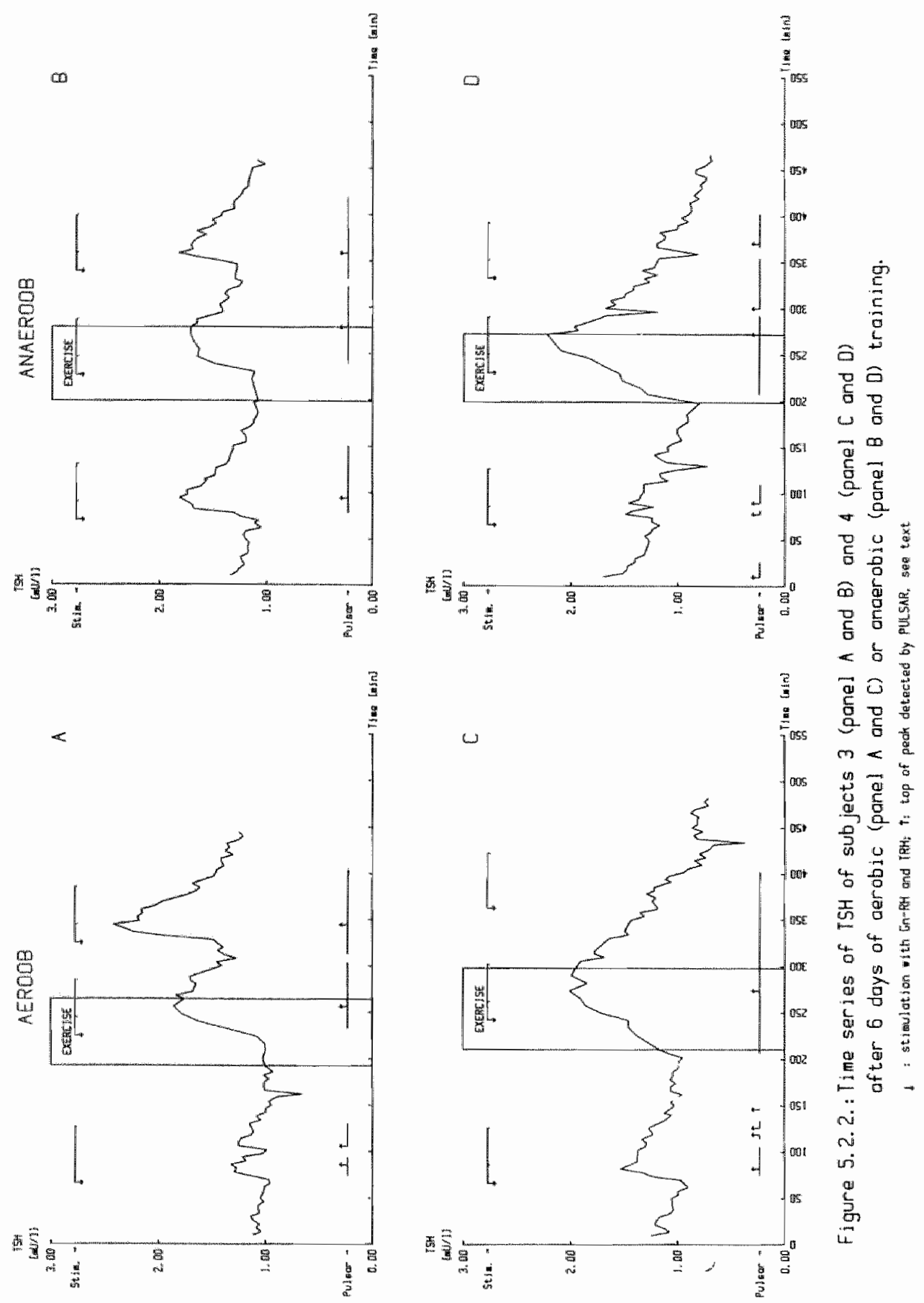

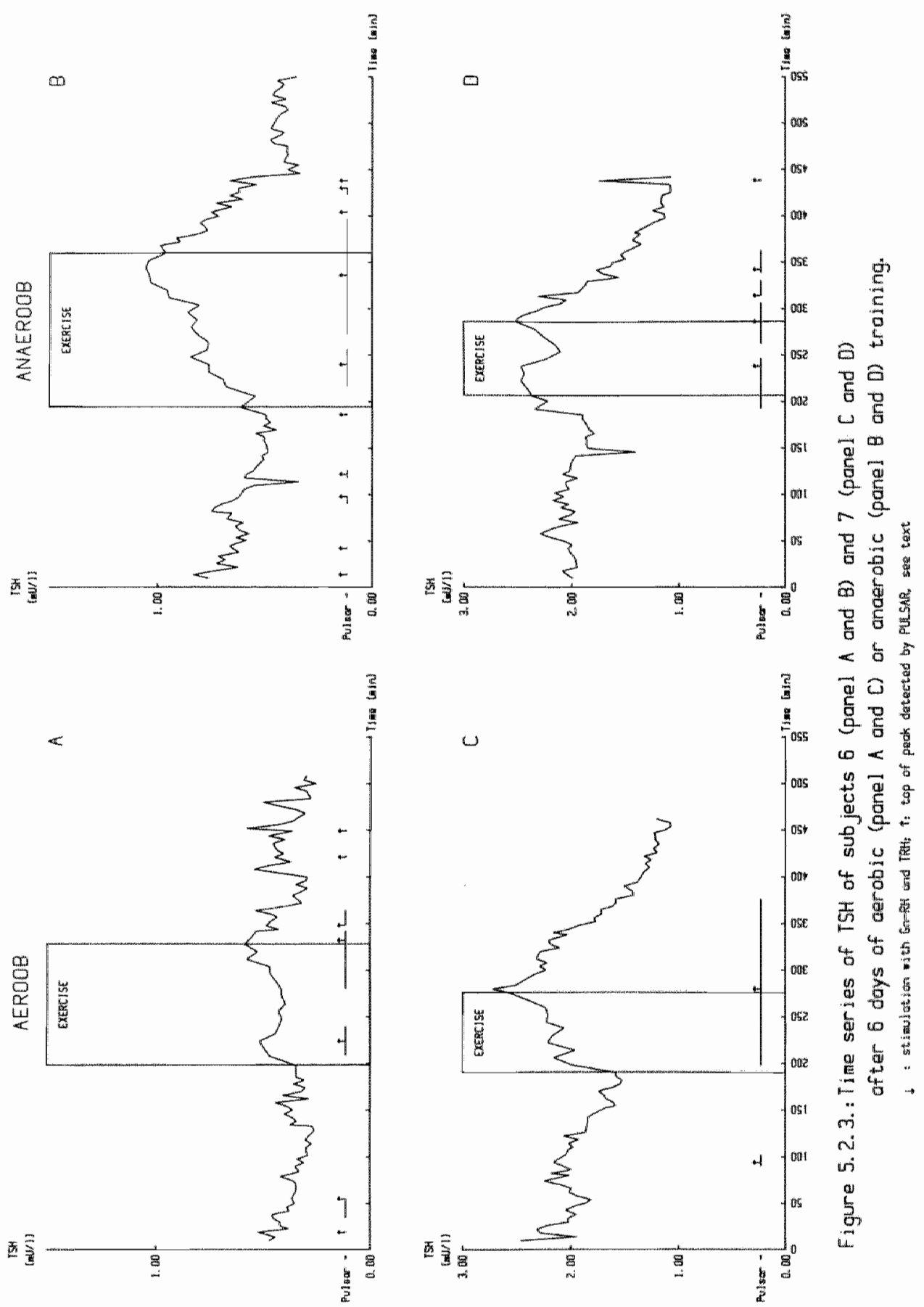

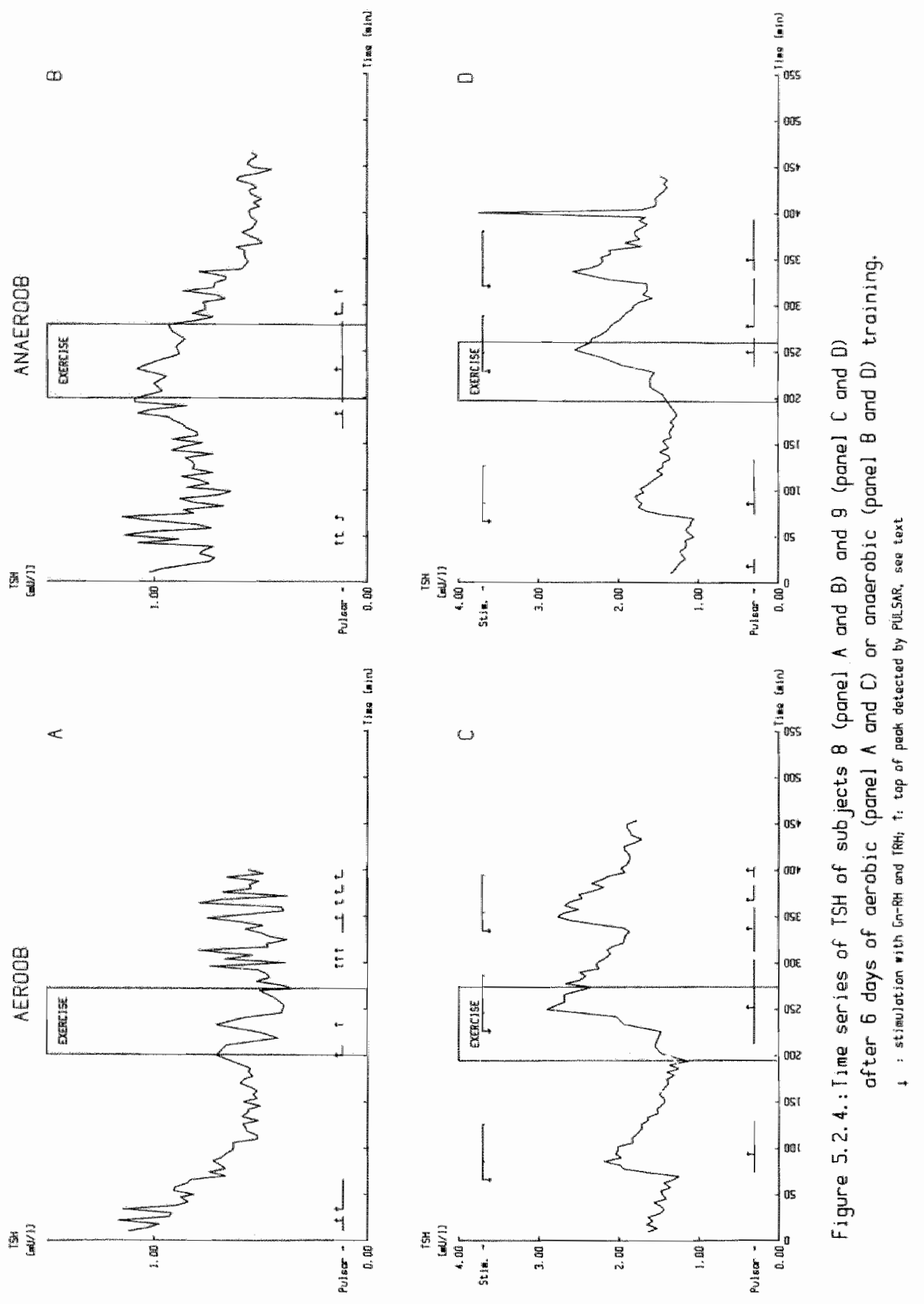

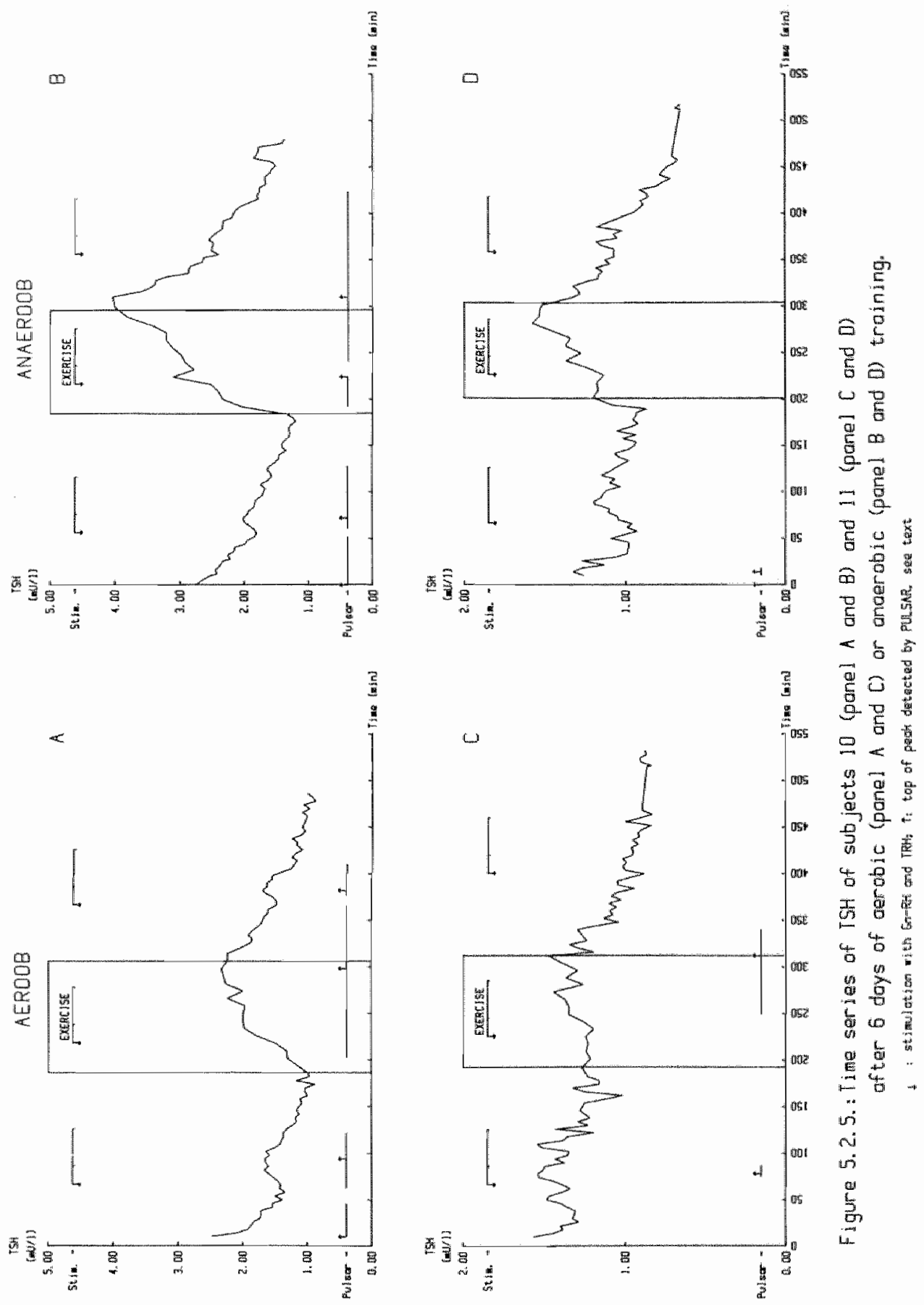

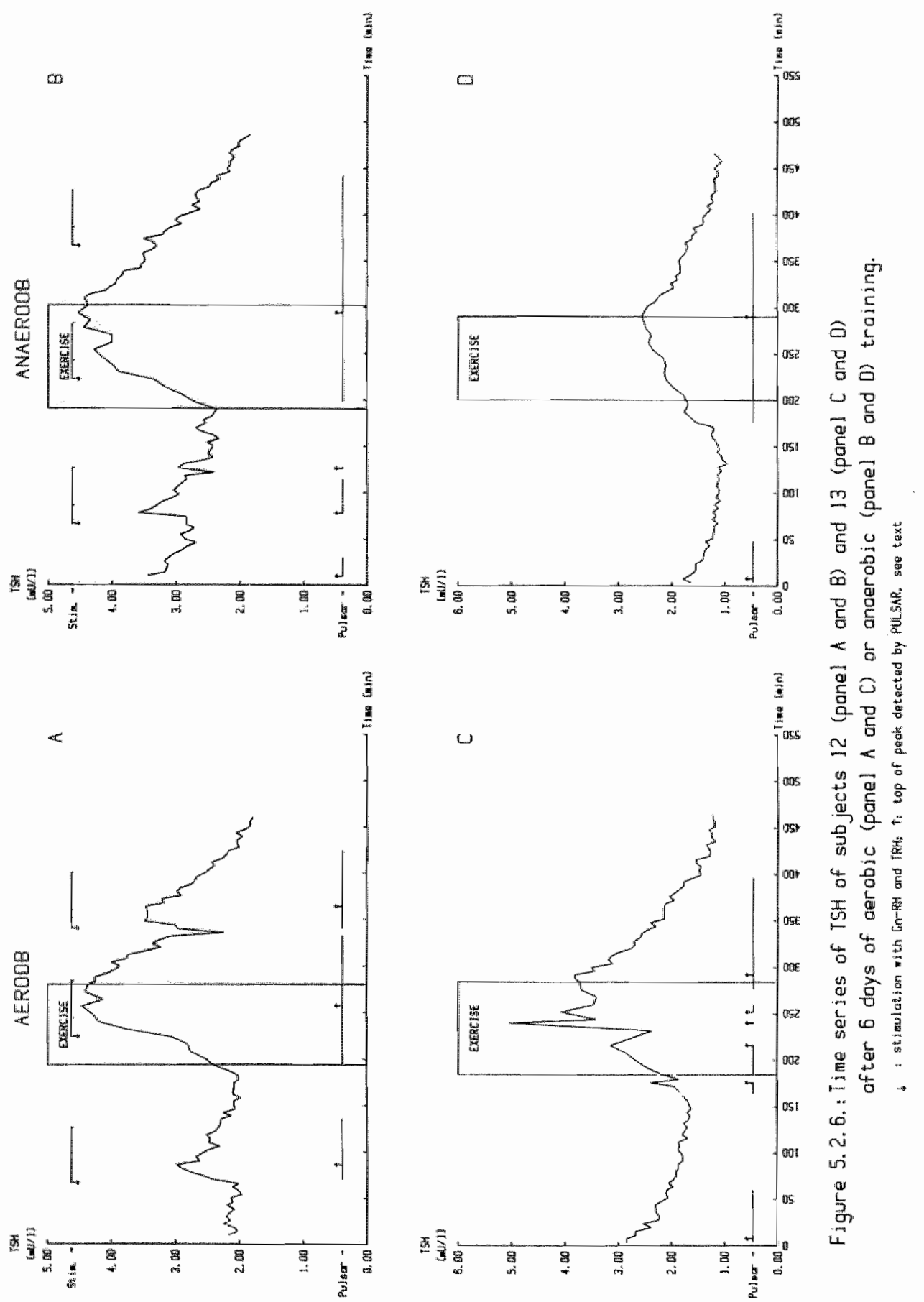

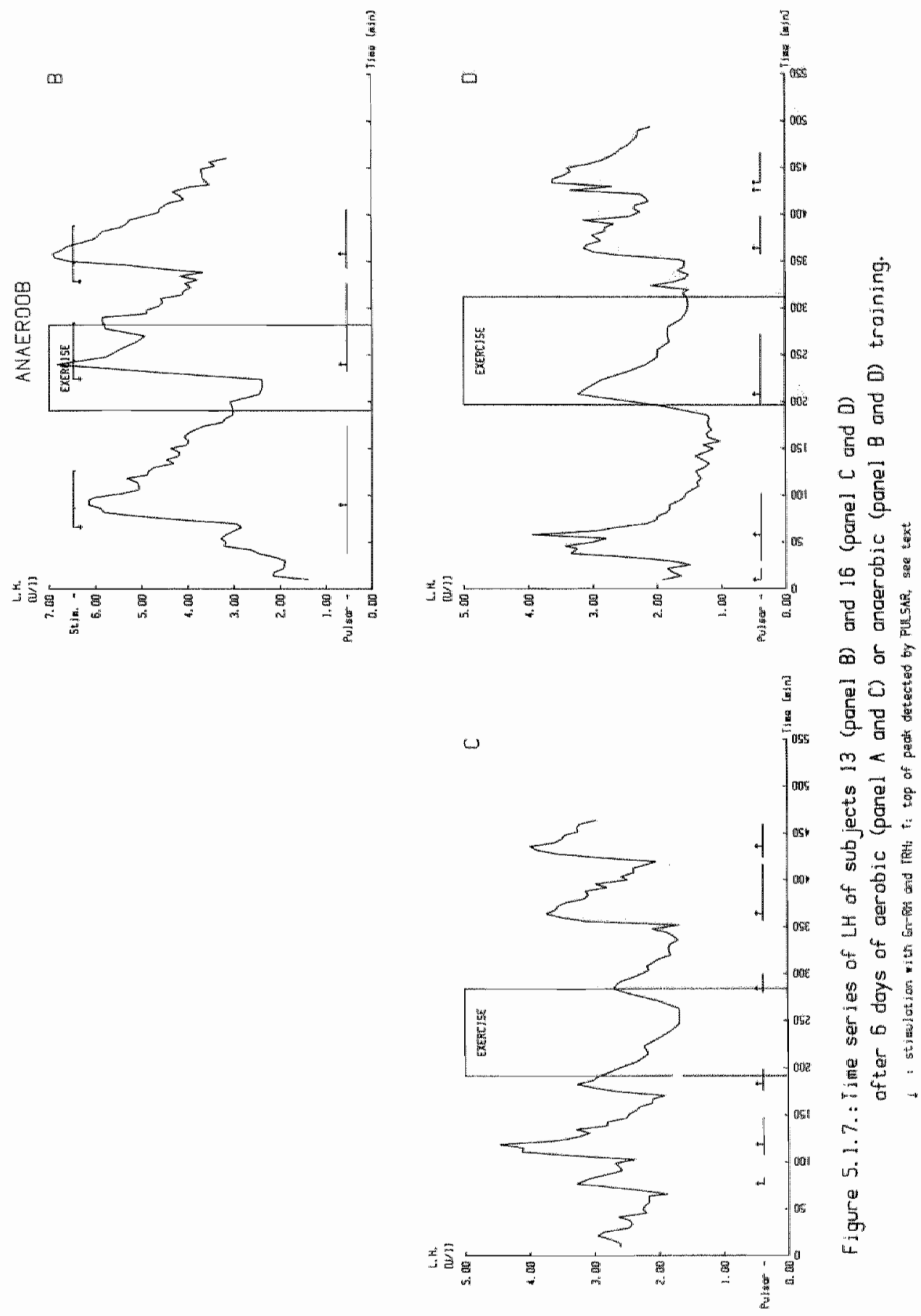


\subsubsection{Discussion}

The results of this investigation show a distinct response of the TSH concentration to acute exercise. This corresponds to the scarcely available literature on this subject (31,33). This reaction upon exercise was present independently from the previous training The intensity of training (aerobic or anaerobic) had no influence on the immediate response of the TSH concentration to exercise. This conflicts with the findings of Schmid et al. (32). They found increased TSH concentrations after submaximal physical exercise. but decreased levels after maximal exercise.

Visual inspection of the pulsatile patterns of the TSH concentrations revealed no changes after exercise in comparison to the pre-exercise period. So, heavy exercise will not influence the pulsatility pattern. This is confirmed by the statistic tests that have been performed on the properties of the PULSAR recognized peaks. Again there are no differences in relation to the character of previous training. This has been found also by Caron et al. (42) in a study of the effect of physical condition on thyroid hormones and TSH. However, Smallridge et al. (43) did not find significant effects of exercise and physical fitness on the plasma TSH concentrations. Due to differences in the experimental design. their results can not be compared to our results. The absence of effects of training on TSH concentrations has been found by more authors $(44,45,46,47,48)$. Again comparisons of these studies with our investigation is impossible due to different protocols. In average we have found 2.01 pulses $/ 3 \mathrm{~h}$. This corresponds to about 16.1 pulses/24h. The mean pulse amplitude was $0.7 \mathrm{mU} / \mathrm{l}$. These figures are in acceptable agreement with literature.

Nevertheless, as general conclusion, we might suggest that it is very unlikely that the intensity of training will influence the thyroid hormone status, which is in agreement with literakure. Studies on the influences of exercise and training on the pulsatility pattern of TSH are not available at all. During the periods of acute exercise, we found (table 5.2.5) that the increase of the TSH concentration expressed as the area under the curve/min just missed significance in stimulated and unstimulated subjects after anaerobic training and in unstimulated subjects after aerobic training in comparison to both the period before and the period after the acute exercise. However, we were unable to demonstrate significant changes in the pulsatility pattern outside the period of exercise. This confirms the absence of changes in TSH-concentration as found in the above studies. 


\section{References}

1: Feight C.B., Johnson T.S. Martin B.J., Sparks K.E. Wagner W.W. Secondary antenomea in athletes.

The Lancet (1978) 2;1145-1146

2: Calabrese L.H., Kirkendall D.T., Floyd M., Rapoport S., Willarns G.W., Weiker G.G. Bergleld J.A.:

Menstrual abnormalities, nutritional patterns and body composition in femate classical baller dancers.

Phys.Sportsmed. (1983) 11;86-98

3: Frisch R.E., McArthur J.W.: Menstrual cycles: Fatness as a determinant of minimum werght for height necessary for their maintenance or onset. Science (1974) 185;949-951

4: Frisch R.E., Wijhak G., Vincent L.: Delayed menarche and amenorhea in baller dancers. New Engl. . of Med. (1980) $303 ; 17-19$

5: Baker E.R., Mathur F.S. Kirk P.F., Landgrebe S.C. Moody L.O., Williamson H.O.: Plasma

gonadotropins, prolactin, and stercid homone concentrations in female runners immedately ahter a long distance run. Fertil.Steril.(1982) 38;38-41

6: Boyden T.W., Pamenter R.W., Grosso D., Stanforth P. Fotkis T., Wilmore J.H.: Protactin responses, mensinual cycles and bady composition of women runners. J.Clin. Endocrinol.Metab. (1982) $54: 711-714$

7: Bullen B.A., Skrinar G.S., Beitins I.Z., Von Mering Z. Tumbull B.A., McArthur J.W.: Induction of mensthat cycte disorders by strenuous exercise in untrained women. N. Engl.J.Med.(1985) 312;1349-1353

8: Shangold M. Freeman R., Thysen B., Galz M.L.: The relationship between long distance running. plasma progesterone and luteal phase tength. Ferti. Sterill,(1979) 31;130-133

9: Keizer H.A., Kuipers H. Haan J.de, Janssen G.M.E. Beckers E., Habets L., Kranenburg G. van, Geurten $P$.: Effect of a 3mmonth endurance training program on metabolic and multiole homonal responses to exercise. Int.J.Sports.Med.(1987) 8;154-160 suppl

10: Bonen A., Keizer H.A.: Pituitary, ovarian and adrenal hormone responses to marathon running.

Int.s.Sports.Med.(1987) 8;161-167 suppl

11: Fries H., Nillius S.J., Peftersan F.: Epidemiolagy of secondany amenornea. Am.J.Obstel.Gyneco. (1974) $118 ; 473-479$

12: Galle P.C., Freeman E.W., Galle M.G. Huggins G.IP., Sondheimer S.J.: Physiologic and psychologic profiles in a survey of women runners. Fertil.Steril.(1983) 39;633-639

13: Chandre A.M., Patra P.B., Chatterjee P., Deb C.: Effect of long term treadmill rurning on gonadal actmity in female rats. Endokrinologie (1978) 72;299-303

14: MCNatty K.P. Sawers R.S., McNeilly A.S.: A possible role for prolactin in control of steroid secretion by the human Graatian follicle. Nature (1974) 250:653-655

15: Santen R.J., Bardin C.W.: Episodic luteinizing hormone secretion in man. Pulse analyses, clinical interoretation, physiological mechanism. J.Clin Invest Metab (1973) 36;55-63

16: Knobil E.: The neuroendocrine control of the menstual cycle. Recent Prog. Horm. Res.(1980) 36;53-88:

17: Boyden T.W., Pamenter R.W., Stantorth P., Rolkis T. Wilmore J.H.: Impaired gonadotropin responses 10 gonadotropin releasing hormone stimulation in endurance trained women. Fertil.Strat (1984) 41:359-363

18: Cumming D.C., Vickovic MM. Wall S.R. Fluker M.P.: Defects in pulsatile LM release in normally menstruating runners. ل. Clin. Endocrinol. Metab.(1985) 60;810-812

19: Cumming D.C. Vickovic M.M. Wall S.F. Fluker M.P., Belcastro A.N.: The effects of acute exercise on pulsatile release of luteinizing hormone in women runners. Am. J Obstet.Gynecol. (1985) 153,482-485

20: Keizer H.A., Bonen A.: Exercise induced changes in gonadotopin secretion panerns.

Int.J.Sports.Med.(1984) 5 suppl;206-208

21: Merriam G.F., Wachter K.W.: Algorithms for the study of episodic hormone secretion.

Am.J.Physiol.(Endocrinol.Metab.6) (1982):E310-E318

22: Veldhuis J.D. Evans W.S. Demers L.M. Thomer M.O., Wakat D., Rogol A.D.: Altered neurcendocrine regulation of gonadorropin secretion in women distance runners. J.Clin. Endocrinol.Metab. (1985) 61:557-563 23: Tepperman J.: Metabolic and endocrine physiology. Year Book Medical Publishers, Inc, Chicago (1974) pp $1-6$

24: Boyden T.W. Pamenter R.W.: Exercise and the thyroid. IN: Fortherby K., Pal S.B.: Exercise

Endocrinology, Walter de Gruyter, Berlin (1985)

25: Metivier G., Gauthier $R$.: The effects of acute exercise on the level of $T_{3}, T_{4}$ and free thyroxine index in the aging male. J.Sports. Med (1981) 21;371-376 
26: Teriung RL. Tipton C.M.; Plasma thyroxine and thyroid stimulating hormone levels during submaxmal exercise in humand. Am. J.Physiol. (1971) 220;1840-1845

27: O'Connell M. Pobbins D.C., Horton E.S., Sirns E.A.H. Dantorth E.Jr.: Changes in serum concentrations of $3,3^{\prime}, 5^{\prime}$-thiodothyronine and $3,5,3^{\prime}$-tritodothyronine during prolonged moderate exercise.

J.Clin Endocrinol Metab.(1979) 49;242-246

28: Kirkeby K, Stromme S.B., Bjerkedal $I_{2}$ Hertzenberg L., Refsum H.E.: Effects of prolonged, strenuous exercise on lipids and thyroxine in serum. Acta Med.Scand.(1977) 202;463-467

29: Theilade P. Hansen J.M., Skovsted L., Kampmann J.P.: Effect of exercise on thyroid parameters and on metabolic clearance rate of antipyrine in man. Acta Endocr.(1979) 92;271-276

30: Retsum H.E, Stromme S.B.: Serum thyroxine, triodothyronine and thyroid stimulating hormone after prolonged heawy exercise. Scan.J.Clin.Lab Invest.(1979) 39;455-459

31: Galbo H. Hummer L., Peterson 1.B. Christensen N.J.H. Bie H.: Thyroid and testicular homone responses to graded and prolonged exercise in man. Europ.J.Appl.Physiol.(1977) 36:101-106

32: Schnic P. Wolf W. Pilger E., Schwaberger G., Pressenholer H., Pristautz H., Leb G.: TSH, T3, TT3 and

17. in meximal and submaximal physical exercise. Europ.J.Appl. Physiol.(1982) 48,31-39

33: Adler J., Brown K., Craig M., Price D.J. Wardlow J.M.: Thyroid function and physical activity.

Br.J.Sports.Med.(1981) $15,261-264$

34: Irvine C.H.G: Effect of exercise on thyroxine degradation in athietes and non-athetes.

J.Clin Endocrinol Metab.(1968) 28;942-948

35: Winder W.W., Heninger R.W.: Effect of exercise on degradation of thyroxine in the rat.

Am.J.Physiol.(1973) 224;572-575

36: Story J.A., Griffith D.R.: Effect of exercise on thyroid hormone secretion rate in aging rats.

Horm Metab.Res. (1974) 6;403-406

37: De Nayer P.H. Astijn M., Visscher M. de: Effet de Ventrainement sur le taux de la thyroxine libre chez l'athlete. Ann.Endocrinol.(1970) 31;721-723

38: Klingler W. Ball P.: Pulsatile, circhorale und circadiane Rhythmik der TSH-Sekretion beim Menschen. NucCompact (1985) 16;433-440

39: Brabant G., Rantt U., Ocran K., Hesch R.D., Muhlen A. von zur: Pulsatile pattern of thyrotropin-release in normal men. Clin.Chim.Acta (1986) 155; 159-162

40: Greenspan S.L., Klibanski A, Schoenteld D. Ridgway E.C.: Pulsatile secretion of thyrotropin in Man. J.Clin.Endocrinol.Metab.(1986) 63;661-668

41: Clark P.M.S. Clark J.D.A., Holder R., Raggatt P.R., Wheatley T.: Pulsatile secretion of TSH in healthy subjects. Ann.Clin.Biochem.(1987) 24:470-476

42: Caron P. . " Sopko G., Stolk J.M., Jacobs D.R. Nisula B.C.: Effect of physical conditioning on measures of thyroid hormone action. Horm. Metab. Res.(1986) 18;206-208

43: Smallidge R.C., Whorton N.E., Burman K.D., Ferguson E.W.: Effects of exercise and physical fitness on the pituitary thyroid axis and on prolactin secretion in male runners. Metabolism (1985) 34;949-954

44: Licala G., Scaglione R., Novo S., Dichiara M.A., Di Vincenzo D.: Behaviour of serum $T_{3}, r T_{3}, T_{4}, T_{4}$ and

TSH levels after exercise on a bicycle ergometer in healthy euthyroid male young subjects.

Baill.Soc.Ital.Biol.Sper.(1984) 60;753-759

45: Opstad P.K, Falch D. Oktedalen O., Fonnum F., Wergeland R.: The thyroid function in young men

during prolonged exercise and the effect of energy and sleep deprivation. Clin. Endocrinoll.(1984) 20;657-669 46: Siddiqui A.R., Hinneleld R.B. Dillon T., Judson W. E.: Immediate effects of heaw exercise on the circulating thyroja homones. Br.J.Sports. Med. (1983) 17;180-183

47: Rolandi E. Reggiani E. Franceschini R., Bavastro G. Messina V., Odaglia G., Barca T: Comparison of pituitary responses to physical exercise in athletes and sedentary subjects. Horm. Res.(1985) $21 ; 209.213$ 48: Sawhiney R.C., Malhotra A.S., Gupta R.B., Rai R.M.: A study of pituitary thyroid function during exercise in man. Indian J.Physiol.Pharmacol.(1984) 28;153158 


\section{Chapter 6:}

\section{General Discussion.}

The development of clinical endocrinology would never have been successful without the simultaneous development of biochemical endocrinology. The opposite is also true: actual knowedge of biochemical mechanisms within endocrine systems would have been composed of assumptions only, if there had been no validation in clinical situations. In this thesis we have attempted to measure small changes in blood glycoprotein concentrations under various (patho)physiological conditions. Until very recently, such small changes in hormone levels would have been obscured by assay noise. The assays we have used were immunoradiometric assays with high sensitivity and specificity.

The increased sensitivity of these new assays permits us to distinguish in much more cletail the differences in behaviour of the pituitary in various clinical conditions, otherwise obscured by a bad signal to noise ratio. The advantages of sensitive TSH assays in the differentiation between patients with hyperthyroidism and euthyroid patients have been largely demonstrated in the literature. The discriminating capacity of sensitive TSH assays is such that they have been proposed as first line tests in thyroid function testing. Even in more complicated cases, such as patients taking amiodarone, sensitive TSH assays have demonstrated that they provide more useful clinical information than all other thyroid function tests (chapter 2).

In chapter 3 of this thesis, the superiority of sensitive TSH assays is demonstrated in patients using ritodrine, in the follow-up of treatment of hypothyroidism and in the study of thyroid function testing in different stages of the menstrual cycle. These subjects have in common, that until now it has been impossible to verify that the clinical condition in these situations depended on changes in thyroid hormone status. The assay of thyroid hormones or of TSH by conventional RIA did not provide decisive information whether or not the thyroid was involved. The use of a sensitive method for the estimation of TSH concentrations appeared to be beneficial, because:

- the use of ritodrine (an agent used in the prevention of preterm labour) in pregnant patients was thought to be complicated in some patients by the development of hyperthyroidism. In fact, increased $T_{4}$ and $T_{3}$ concentrations, combined with increased heart frequencies suggested hyperthyroidism. In our study, we have shown that the change in $T_{3}$ concentrations, undoubtedly contributing to tachycardia, is only temporarily In only 1 out of 17 patients was the TSH concentration suppressed after initiating therapy The estimation of TSH with a sensitive method permitted the conclusion that tocolysis therapy is unlikely to contribute to the development of hyperthyroidism.

- homeostasis of thyroid hormones is regulated in a very precise manner. The intra-individual variation is much smaller than the variability between individuals. For this reason and also because of assay imprecision itself, it is difficult to interpret small changes in thyroid hormone concentrations. The concentration of TSH reflects the set-point of the pituitary regulation. Because of the feedback mechanism, changes in thyroid hormone concentrations are amplified in the accompanying changes in the concentration of TSH. However, slight variations in euthyroid subjects are still too small to 
be detected by insensitive TSH assays. Using a sensitive assay we measured variations in thyroid status belore and after ovulation. In all but one subject, TSH was found to decrease after ovulation. In the one subject who showed a (non significant) increase, no ovulation had occurred. We suggest that the decrease in TSH concentration reflects a slight increase of the free thyroxine concentration, possibly due to displacement of thyroxine from the binding proteins by progesterone.

- patients receiving 1-thyroxine replacement therapy, as in hypothyroidism, can be monitored in various ways. It seems logical to use end-organ responses such as PEP/LVET or SHBG in addition to the biochemical parameters $T_{4}, T_{3}$, free $T_{4}$ and $T S H$. For various reasons, over-or undertreatment with l-thyroxine needs to be detected and corrected. However, in our study none of the above parameters gave a specificity and sensitivity of $100 \%$ with regard to the detection of over-or undertreatment. The end-organ parameters showed little correlation with clinical status, just as with TSH. Nevertheless, since it is known that TSH is a sensitive marker of dysthyroidism, the latter parameter is preferred. If restoration of entirely normal TSH levels is not desired, for example in hypothyroid patients with non-thyroid dependent heart disease, monitoring of therapy by the assay of TSH can be combined with subjective clinical judgement in order to economize heart activity.

In conclusion, the use of sensitive assays for the estimation of TSH permits a refinement in the study of thyroid status in various clinical conditions

Periodic variations within the dynamic processes of life are believed to contribute to the adlaptive capacities of living beings to external influences. In chapter 4 , a brief survey of literature of biorhythms is presented. In humans, rhythms within the endocrine system can vary over minutes to months. Examples of this are TSH and LH (minutes), cortisol (daily), menstrual cycle (weeks) and thyroxine (seasonal). The periodicity of the rhythms may vary with age. It is evident that the apparent periodicity of the hormone concentrations in plasma greatly depends on the half-life: it is useless to search for pulsatile patterns in the plasma concentrations of those hormones that have a half-life of more than one hour. The properties of feedback systems necessitate a continuous increase and decrease of the 'key'-hormones within an endocrine regulatory system. Mathematically, pulsatile patterns can be expected for the stimulating hormones (e.g. TSH, LH and perhaps FSH) and for the releasing hormones (e.g. TRH and $\mathrm{Gn}-\mathrm{RH}$ ), i.e. those hormones that have short half-lives. Biologically, it appears that pulsatile behaviour is necessary: continuous suppletion of the hormones that normally show a pulsatile pattern leads to an antagonistic function. It is not surprising that the episodic paltern of secretion of pituitary hormones has been described only sparsely in the literature. The variations are so small that they are undetectable without sensitive methods of estimation. Moreover, pituitary hormones are structurally closely related. This lays particular emphasis on the specificity of the assays. In the past, pulsatility studies could only be performed with those hormones that show large pulse amplitude, e.g. LH. Only recently have a limited number of studies on TSH pulsatility been carried out.

Due to the absence of sensitive assays, few pulsatile studies have been performed in the past and in consequence, the methodology of pulsatility study is at the first stage of development. Much has to be done to improve, for example, the sampling procedures and the algorithms of peak detection. The assay methodology should be thoroughly evaluated whether or not sensitivity and specificity satisfy the needs of the special 
character of such studies. The specificity of the assays has to be verified to correlate with in-vivo activity of hormones. Sensitivity must be improved in such a way in order that the assessment of pulsatility of pituitary hormones really opens the possibility of judging hypothalamic activity. Since pulsatility is thought to diminish with age, future developments undoubtedly will be important in the study of aging and perhaps may contribute to a better understanding of the processes of life. In this respect, a better understanding of the hypothalamic mechanism for controlling pituitary activity might be beneficial. The study of pulsatility of pituitary hormones offers one of the rare possibilities of approaching the hypothalamus. However, it must be realized that at this moment the actual lack of knowledge will prevent definite conclusions with regard to hypothalamic function being drawn from results of pulsatility studies.

In chapter 5 the results of pulsatility studies of TSH and LH are presented. Since physical exercise is involved in many' endocrine functions, these studies have been performed using chronic and acute exercise as triggers of hypothalamic activity.

It was demonstrated that pulsatility of $\mathrm{LH}$ did nott change in response to acute exercise. Furthermore, training (chronic exercise) influenced neither pulsatility nor basal hormone concentrations. Although in most of the literature it is suggested that the disturbances observed in the pulsatility pattern of $\mathrm{LH}$ are responsible for the changes in menstrual cycle in response to training and exercise, we were unable to demonstrate such relation. The other hormone studied, TSH, showed a small increase in response to acute exercise. Again, previous training influenced neither the pulsatility pattern nor the basal hormone concentration. The function of the increase in TSH level in response to acute exercise has to be established. It is not likely that exercise results in a changed thyroid status, but our results are too preliminary to be decisive.

One of the merits of modern medicine is the possibility of dealing with problems that cause discomfort rather than being the source of morbidity. Infertility is such a problem, while the prevention of osteoporosis in cases of amenorrhea is another one. Chronic exercise may result in oligo- or amenorrhea with possibly an increased chance of the development of the above problems. This study has shown that physical training, although at a recreational level, may lead to a significantly lengthened menstrual cycle. However, we were unable to link this observation to altered LH pulsatility. Partly this may be due to the absence of large number of subject data with proven menstrual cycle normality, In addition, the study of the behaviour of prolactin in response to exercise may be more conclusive. This can be expected, since there is a good correlation between the secretion of TSH and prolactin by the thyrotrophic cell. Another possibility to explain the menstrual disturbances can be a changed behaviour of the ovarian inhibin production in response to exercise. Finally, attention must be directed to the most appropriate moment of sampling in pulsatility studies: at other stages (non-mid folicular) of the menstrual cycle the results may have been completely different.

In conclusion, the sensitive and specific estimation of pituitary glycoproteins proves to be the most important condition in studies of pituitary and hypothalamic activity. Further developments are needed in order to delineate their place in daily routine. Undoubtedly, such methods will be of value in applications that satisfy the human desire for a better quality of life. 



\section{Summary}

In this thesis the features of sensitive estimations of pituitary glycoprotein hormones are described, together with some clinical and scientific applications.

After a general introduction, the first chapter deals with the important role of hormones in the regulation of many metabolic processes. The central role of the pituitary as a controiler in many endocrine processes is discussed. Influenced by higher brain centres such as the hypothalamus, pituitary hormones are secreted into the bloodstream through which they are transported to the specific target organs where the hormone activity is displayed Thyroid stimulating hormone (TSH) is one of these pituitary glycoproteins. It stimulates the thyroid into production and secretion of thyroid hormones: thyroxine $\left(T_{4}\right)$ and triiodothyronine ( $\left.T_{3}\right)$. Another pituitary glycoprotein is the luteinizing hormone $(\mathrm{LH})$. It is responsible for the process of ovulation in women and for the stimulation of Leydig cells leading to the production of testasterone in men. A negative feedback system exists, meaning that an increased concentration of target organ hormones (such as hormones of the thyroid, the ovaries and the testes) inhibits the production and secretion of the stimulating hormones such as TSH and LH. Even very small quantities of glycoprotein hormones are sufficient to stimulate target organs. In order to measure the pituitary glycoprotein concentrations in the plasma, ultra sensitive methods for determination are required. The radioimmunoassays generally used (RIA techniques, until 1984) were quite adequate for measuring normal or elevated concentrations of stimulating hormones but decreased values could not be measured with any accuracy. However, measurement of decreased values now appears to be quite important in the study of endocrine pathophysiology.

In chapter 2, a description is given of ultrasensitive immunoradiometric assays (IRMA techniques, available since 1984). By application of these IRMA methods, detection limits 10 to 50 times lower than those for RIA methods can be reached. In this chapter the theoretical background of these improved methods and their inherent pitfalls are discussed. It has also been pointed out that perhaps even lower detection limits could be reached using other labels than the generally available ${ }^{125}$. The studies in this thesis, however, have been performed using ${ }^{125}$ lodine-labelled tracers. Commercially available reagents have been compared both for TSH and LH estimations, hitherto the only avalable ultrasensitive procedures, and the analytical accuracy and precision have been determined.

Some clinical applications of the ultrasensitive TSH determinations have been described in chapter 3. A number of publications have appeared indicating the possibilities of IRMA-TSH for the measurement of decreased concentrations such as found in hypothyroidism. The IRMA-TSH determination is now very important in the diagriosis of thyroid hormone abnormalities. Moreover, there is now the possibility of following even very small concentration changes, which could not be distinguished using the earlier FilA techniques but were lost in the bias of the analyses. The importance of such small changes is elucidated in chapter 3.1 studying the effects of a B-mimetic (ritodrine) on the thyroid hormone status. This drug is used in suppression of early labour during pregnancy. It has been suggested that hyperthyroidism could be induced as a 
complication. In our investigations an elevation of the $T_{3}$ concentration of short duration has been found. Most probably this is a reason for the tachycardia which is often observed as a side effect of administration of ritodrine. During this type of therapy only one of seventeen patients showed suppression of TSH levels in the blood, compatible with hyperthyroidism. In 16 other patients, TSH concentrations correlated well with reference values for euthyroidism. It can be concluded from our observations that the use of ritodrine leads to temporary stimulation of a de-iodase enzyme (type I de-iodase). responsible for the catabolism of thyroid hormones. Ritodrine itself does not induce the development of hyperthyroidism. In hyperthyroid patients, however, an increased conversion of $T_{4}$ to $T_{3}$ must be considered.

In chapter 3.2 the ultrasensitive TSH determination is used for studying the changing thyroid status after ovulation. It appears that when compared to the follicular phase, the TSH concentration after ovulation is slightly decreased. In a non ovulating patient no decrease of TSH concentration has been found. AS TSH is produced by the pituitary and is subject to a negative feedback mechanism by thyroid hormones, the decrease of TSH concentration must be the result of increase of thyroid hormones in the blood. This latter increase is so small that it can not be observed using the avallable determination methods. Moreover, probably only the concentration of non-protein-bound thyroid hormone is changed. This can be explained by the hypothesis that the free thyroid hormone concentration increases by displacement of thyroxine from the binding proteins by the considerably elevated progesterone concentration in the blood during the luteal phase of the menstrual cycle.

In chapter 3.3 the suitability of the ultrasensitive TSH determination for the follow up of patients on synthetic thyroid hormone therapy is described. The regulating system, including the feedback mechanism for TSH secretion, is kept upright and even during subfunction of the thyroid gland the TSH level in the blood is a parameter for the correct suppletion of exogeneously administered thyroxine. This thyroxine suppletion could also be controlled by functional parameters such as the PEP/LVET ratio or the SHBG concentration. The underlying idea is that peripheral effects of thyroid hormones are more important in substitution therapy than the effects on the pituitary-thyroid and hypothalamic-pituitary stimulating systems. We have shown, however, that these functional parameters have little correlation with the clinical symptoms of the patients. The same is true for the plasma concentrations of the thyroid hormones and of TSH. Sensitivity and specificity of all determined parameters for the assessment of the euthyroid status appear to be relatively low. As there is no 'golden standard' for euthyroidism, however, the TSH determination with the good discrimination possibilities for comparison of hyperthyroidism and euthyroidism seems to be the method of choice for the control of thyroid hormone suppletion.

It can be concluded that the ultrasensitive TSH determination introduces a refinement in the diagnosis and treatment of abnormal thyroid hormone conditions.

The second pituitary hormone which has been investigated is the luteinizing hormone (LH). Ultrasensitive detection and determination methods are now available for this glycoprotein. Very few clinical applications have been suggested, but the LH determinations seem to be quite valuable for the study of biorhythms in response to external changing situations such as stress and physical exercise.

In chapter 4 a short survey is given of biorhythms in endocrinology. It appears that 
changes in hormone levels are mostly so small that they can only be detected with ultrasensitive methods. Moreover, there is great chemical similarity between different pituitany hormones and determination methods have to be very selective as well. The IRMA methods (as described in chapter 2) appear to be suitable for this type of investigation. However, more information must be obtained concerning differences between biological and immunological/immunochemical activities of the glycoprotein hormones: this is especially true for $\mathrm{LH}$.

One of the ultrasensitive LH determinations now available has been used to study the pulsatile character of pituitary hormone secretion. This pulsatility is thought to be a reflection of hypothalamic activity in which the hypothalamus is considered to connect the neurogenic and endocrine regulation systems.

Pulsatility studies of protein hormones have only just started, and the lack of sensitive and specific analytical procedures was one of the reasons for this. Studies on the influence of physical exercise, both training and acute exercise, on the endocrine system have nevertheless been performed. Therefore physical exercise as a trigger of the endocrine system in pulsatility studies was chosen for our investigations as described in chapter 5 . We have seen that the pulsatile properties of LH does not change under acute physical exercise. It also seems that chronic exercise (training) has no influence either on the pulsatility or on the basal hormone concentrations. However, physical exercise leads to prolongation of the menstrual cycle in healthy women. It is now clear that this can not be explained by changing $\mathrm{LH}$ concentrations or changing pulsatile secretion.

A slight elevation of the TSH concentration in the plasma has been observed with acute physical exercise. The pulsatile character of TSH secretion is not influenced and the basal hormone concentration shows no change.

We have shown that in modern medicine many problems can be studied that are not directly related to disease, but more to the quality of life. The development of diseases can be determined in an earlier stage. Both possibilities are due to improvement and development of highly advanced analytical techniques as a part of refined diagnostic procedures. Molecular biology is the basis of physiology and disease, and research in this field will favourably influence clinical diagnosis and treatment. 



\section{Samenvatting}

\section{Gevoelige bepalingen van hypofysaire glycoproteine hormonen: klinische en natuurwetenschappelijke toepassingen.}

Dit proefschrift handelt over gevoelige eiwithormoonbepalingen en hun toepassingen.

In hoofdstuk 1 wordt aangegeven, hoe belangrijk hormonen zijn in de regulatie van vele processen, die zich in het lichaam afspelen. De hypotyse (een orgaan van ongeveer 10 * 13 * 6 mm met een gewicht van 0.5 gram, gelegen in de 'sella turcica' in de schedelbasis) is het centrale regelorgaan voor de huishouding van vele hormonen. Onder invloed van de hersenen worden hormonen aan de bloedbaan afgegeven, die elders in het lichaam hun werking uitoefenen op zogeheten doelwitorganen. Het TSH (Thyroid Stimulating Hormone, schildklier stimulerend hormoon) is een van die hormonen. Het stimuleert de schildklier tot de productie en secretie van de schildklierhormonen: thyroxine $\left(T_{4}\right)$ en trijodothyronine $\left(T_{3}\right)$. Een ander hypofysair hormoon, het luteinizerend hormoon of $\mathrm{LH}$, is verantwoordelijk voor het tot stand komen van de ovulatie bij de vrouw en de stimulatie van de Leydigcel tot de productie van testosteron bij de man. Er is een negatieve terugkoppeling naar de hypofyse, hetgeen betekent dat hoe hoger de concentratie van de hormonen, zoals die door de doelwitorganen (schildklier, ovarium en testis) uitgescheiden worden, hoe lager de concentratie van de stimulerende hormonen TSH en LH. Slechts zeer geringe hoeveelheden hormoon zijn nodig om de doelwitorganen te stimuleren. Er moeten dan ook gevoelige bepalingsmethodieken gebruikt worden om deze concentraties in plasma te kunnen meten. Tot 1984 hadden de gebruikte bepalingsmethodieken (RIA- technieken) het nadeel dat zij wel de normale of verhoogde concentraties van de stimulerende hormonen konden meten, maar niet die verlaagde concentraties.

Deze zijn echter in de praktijk toch ook erg belangrijk.

In hoofdstuk 2 wordt een beschrijving gegeven van de IRMA- technieken, zoals deze voor de bepaling van de eiwithormonen sinds 1984 worden gebruikt. De IRMA-methodiek is 10 tot 50 keer zo gevoelig als de RIA-methodiek. In dit hoofdstuk wordt ingegaan op de theoretische achtergronden van deze verbetering. Verder wordt aangegeven dat de detectiedrempel wellicht nog verder zal dalen, indien overgestapt wordt op andere labels dan ${ }^{125} \mathrm{~J}$. De experimenten voor dit proefschrift zijn evenwel nog uitgevoerd met behulp van tracers die met dit isotoop gelabeld waren. De commercieel beschikbare methodieken voor zowel TSH als $\mathrm{LH}$ zijn met elkaar vergeleken en de analytische juistheid en nauwkeurigheid werden vastgesteld.

In hoofdstuk 3 worden enige klinische toepassingen van de zeer gevoelige TSH bepaling beschreven. 
Er zijn artikelen verschenen, waarin de aandacht wordt gevestigd op de mogelijkheden die de IRMA-TSH biedt am verlaagde concentraties (zoals die voorkomen bij bijvoorbeeld hyperthyreoidie) en normale concentraties te kunnen onderscheiden. Hierdoor heeft de IFMA.TSH bepaling een vooraanstaande plaats gekregen in de diagnostiek van schildklierafwijkingen. Doordat de bepaling van TSH zoveel gevoeliger is geworden is hel ook mogelijk om kleine veranderingen in concentratie te kunnen detecteren. Het gaat dan over veranderingen, die zo klein zijn dat zij vroeger in de ruis zouden zijn verdronken. Dat deze toch belangrijk kunnen zijn, wordt in hoofdstuk 3.1 aangetoond, bij de bestudering van de effecten van een bepaald geneesmiddel op de schildklierhormoonstatus. Daarbif gaal het om ritodrine, een stof die vroegtijdige weeën bij zwangere vrouwen kan onderdrukken. Als bijwerking bij gebruik van dit middel zou hyperthyreoidie kunnen ontstaan. Het onderzoek heett aangetoond, dat er een kortdurende verhoging van de $T_{3}$ concentratie is. Deze is ongetwifeld verantwoordelijk voor het optreden van tachycardie, zoals dat vaak bij het gebruik van ritrodine gezien wordt. Slechts 1 van 17 patienten bleek tijdens de therapie een TSH concentratie te hebben, die overeenkomt met een hyperthyreoidie. Alle andere patienten hadden een TSH concentratie binnen de referentiewaarden voor euthyreoidie. Op grond van de waarnemingen wordt geconcludeerd, dat het gebruik van ritodrine leidt tot tijdelijke stimulatie van een van de dejodase enzymen (het type I dejodase) die verantwoordelijk zijn voor de omzetting van de schildklierhormonen naar atbraakproducten. Ritodrine zelf geeft dus geen aanleiding tot de ontwikkeling van een hyperthyreoidie; wel moet er bij hyperthyreotische patienten rekening gehouden worden met een versterkte omzetting van $T_{4}$ naar $T_{3}$. in hoofdstuk 3.2 wordt gebruik gemaakt van de zeer gevoelige TSH bepaling om de verandering van de schildklierstatus na de ovulatie te bestuderen. Het blijkt dat in vergelijking met de folliculaire fase de TSH concentratie na de ovulatie licht gedaald is. Bij een patiente die niet ovuleerde, bleek er geen daling van de TSH concentratie te zijn. Aangezien het TSH geproduceerd wordt door de hypofyse, terwill de hypofyse negatief terug gekoppeld is met de schildklier, moet de daling van de TSH een afspiegeling zijn van een verhoging van de schildklierhormoonconcentratie. Deze verhaging is echter zo gering, dat deze met de huidige bepalingsmethoden niet gemeten kan worden.

Waarschijnlijk is bovendien alleen de concentratie vam het schildklierhormoon in vrije vorm (niet aan eiwit gebonden) veranderd. De vrije-thyroxine concentratie kan enigszins verhoogd zijn door verdringing van eiwit gebonden thyroxine door de verhoogde progesteron concentratie in de luteale tase.

In hoofdstuk 3.3. wordt beschreven, dat de gevoelige TSH bepaling ook gebruikt kan worden voor het volgen van patienten die synthetisch schildklierhormoon krijgen vanwege een hypothyreoidie. Het regelsysteem van de TSH secretie bliff dan in stand, waardoor na het uitvallen van de schildklier middels de TSH bepaling gekeken kan worden of de suppletie met thyroxine adequaat is. Deze suppletie zou ook gecontroleerd kunnen worden met functionele parameters, zoals de PEP/LVET verhouding of de SHBG concentratie. Dit laatste stoelt op de gedachte, dat eerder de perifere effecten van de schildklierhormonen gemeten zouden moeten worden, dan de effecten ervan op het regelsysteem binnen de hypofyse. In het onderzoek bleek, dat de functionele paramelers weinig correlatie vertonen met de klinische indruk, die de patienten maken. Hetzelfde geldt ook voor de concentraties van de schildklierhormonen en van het TSH. De gevoeligheid en de specificiteit van alle bepaalde parameters voor het vaststellen van een 
euthyreotische status blikt niet groot te zijn. Er is echter geen "gouden standaard", die zegl wanneer een bepaalde patient euthyreoot is. Op grond van het grote onderscheidings vermagen van de TSH bepaling ten aanzien van de hyper/euthyreotische status, wordt de suggestie gedaan de suppletie van schildklierhormoon te controleren met behulp van deze bepaling.

Samenvattend kan dus gesteld worden, dat de gewoelige TSH bepaling een verfining mogelijk heeft gemaakt binnen de detectie van afwijkingen van de schildklierhormoon status.

Ook het tweede hypotysaire hormoon dat is onderzocht, het luteinizerend hormoon, kan tegenwoordig bepaald worden met gevoelige en specifieke bepalingsmethodieken. Hiervoor zijn echter nog maar heel weinig klinische toepassingen bekend. Wel kan juist dit hormoon bepaald worden, wanneer men de periodieke veranderingen in het lichaam wi bestuderen, die modig zijn om het lichaam voortdurend aan te passen aan veranderde omstandigheden, zoals stress na en tijdens lichamelijke inspanning. In hoofdstuk 4 wordt een kort overzicht gegeven van de bioritmes die binnen de endocrinologie bekend zijn. Het blijkt dat de veranderingen dusdanig gering zijn, dat zij alleen met zeer gevoelige methoden kunnen worden vastgesteld. Bovendien lijken de hormonen die door de hypofyse worden geproduceerd chemisch zo veel op elkaar, dat de bepalingsmethodiek ook zeer specifiek moet zijn. Met de in hoofdstuk 2 beschreven IRMA methodieken, blijkt de ideale bepalingsmethoden dicht te zijn benaderd. Wel blijven er nog vragen over ten aanzien van verschillen tussen de biologische en de immunologische activiteit van het bepaalde hormoon, hetgeen met name van toepassing is bij het LH.

Een van de beschikbare gevoelige LH-methodes is gebruikt om de pulsatiliteit van de hypofysaire secretie te bestuderen. Deze pulsatiliteit word beschouwd een afspiegeling te zijn van de hypothalame activiteit, waarbij de hypothalamus gezien wordt als de verbindingsplaats tussen neurogene en endocriene regelsystemen.

De studies naar pulsatiliteit van eiwithormonen staan echter nog in de kinderschoenen, onder andere omdat tot nu toe gevoelige en specifieke bepalingsmethoden ontbraken. Wel is het een en ander bekend over de invloed van fysieke belasting (training en acute inspanning) op het endocriene systeem. Daarom is juist fysieke inspanning gekozen als trigger van het endocriene systeem bij de pulsatiliteitsstudies van hoofdstuk 5 . Wii hebben aangetoond dat de pulsatiliteit van het $\mathrm{LH}$ niet verandert onder invloed van acute inspanning. Verder is gebleken, dat chronische inspanning (training) ook geen invloed heeft op de pulsatiliteil of op de basale hormoonconcentratie. Wel bleek dat inspanning leidde tot verlenging van de menstruele cyclus bij de proefpersonen. De verklaring hiervoor moet dus niet in veranderde LH concentraties gezocht worden. Acute inspanning gaf well aanleiding tot een (geringe) verhoging van de TSH concentralie. Ook hier echter kon aangetoond worden, dat het pulsatiliteitspatroon niet was beinwoed en ook dat de basale hormoon concentratie niet veranderde.

De moderne geneeskunde is in staat problemen aan te pakken, die niet meer rechtstreeks met ziekte te maken hebben, maar die wel met de kwaiteit van het leven van doen hebben. Ook worden ziekten zelf in een steeds eerder stadium gediagnosticeerd. Deze ontwikkelingen zijn mogelijk geworden door een verfijning van de diagnostische methoden, waarbij de ontwikkeling van ultrasensitieve, specifieke analyses een essentiele roil speet. 



\section{Dankwoord}

Vele vruchtbare discussies hebben de bron gevormd, waaruit dit proefischrift is ontsprongen. Allen, die op enigerlei wijze aan de tot stand koming ervan hebben bijgedragen, wil ik dan ook van harte bedanken. Enkelen thebben daarbij echter zo'n dominante rol gespeeld, dat ik mij verplicht voel hen expliciet te noemen:

Met groot plezier wil ik dan ook de zeergeleerde Dr.H.A. Keizer van harte bedanken. Beste Hans, je hebt mij bekend gemaakt met onderzoek naar pulsatiliteit bij de secretie van eiwithormonen. Vruchtbare contacten met andere research centra zijn daaruit voortgewloeid. Je hulp daarbij was onontbeerlijk: ik heb je daarbij ervaren niet als stok achter de deur, maar als stimulator; niet als betweter, maar als discussiepartner. Ik was geen passagier bij het onderzoek, maar medebestuurder. Mijn waardering voor dit vertrouwen.

Hooggeleerde Prof.Dr.P.J.Brombacher, beste Paul, 10 jaar geleden heb je mil op het spoor gezet van de immunochemie; een techniek die toen nog in de kinderschoenen stond. Het is mij dan ook een groot genoegen geweest om nu met een onderzoek naar toepassingsmogelijkheden van een evidente verbetering binnen die immunochemie bij je terug te komen met zelts een promotieonderzoek. Mijn hartelijke dank voor de hulp en de stimulans, die ik daarbij van je mocht ontvangen.

Hooggeleerde Prof.Dr.W. van der Slik, beste Wim, je hebt aan de wieg gestaan van het onderzoek met gevoeliger methodieken. De wijze, waarop je mij in Groningen hebt opgevangen en begeleid, heb ik ten zeerste gewaardeerd. Ik ervaar het dan ook als een grote eer, dat je tweede promator hebt willen zijn.

Hooggeleerde Prof.Dr.J.A. Flendrig, beste Guus, graag wil ik je bedanken voor de opbouwende kritiek die ik van je op het manuscript mocht ontvangen. De snelheid

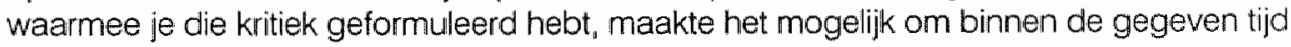
het manuscript bij te schaven tot een beter begrijpbaar geheel.

Sehr geehrter Prof.Dr.Dr.H.Greiling, Ich möchte Sie gerne danken für den Empfang auf Ihrem Laboratorium. Ich danken Ihnen für das Lesen des manuskripts und Ihre positive Bemerkungen.

Hooggeleerde Prof.Dr.R.S. Reneman, beste Rob, graag wil ik je bedanken voor jouw oordeel over het manuscript. Het heeft ongetwiffeld geleid tot aanpassingen "die het geheel leesbaarder gemaakt hebben.

Dear Prof.Dr.J.D. Veldhuis. I am very indebted to you for various reasons. Firstly, you took the time necessary to introduce me the techniques and quality controll of pulse detection during a congress in Paris two years ago. Secondly, this discussion continued the last years thanks to modern methods of communication and lastly, you honered me by coming to Maastricht. I hope this cooperation will be continued in a fruitful manner in the future.

Zeergeleerde Dr.J.LH Evers, Zeergeleerde Dr.A.C. Nieuwenhuyzen Kruseman. Beste Hans, beste Arie, zonder enige verplichting zijn jullie zo bereidwillig geweest om het manuscript kritisch te lezen en van kanttekeningen te voorzien, waardoor het veel aan waarde gewonnen heeft. De samenwerking tussen ons is een schoolvoorbeeld van de 
relatie die er overal tussen kliniek en laboratorium zou moeten zijn.

Zonder meetresultaten zou dit proefschrift zonder waarde geweest zijn. De analyses werden met groot enthousiasme en onvolprezen accuratesse uitgevoerd door Sylvia Jackle-Beckers, Hanneke van Berlo, Harry Hamers, Josée Houben, Peter Gunther, Serva Rozenhout, Astrid Steyns, en Jos. Wiersma onder de bezielende leiding van Marle-Josée Vranken-Vranken. Ook wil ik graag Peter Geurten en Gerrit van Kranenburg bedanken voor hun hulp bij het uitsplitsen van de monsters. Geehrte Dr.P.Platen. C.Peters, R.Biwer und H.Prinz, lch danke euch für den grossartigen Einsatz im LH/TSH Versuch. Ohne euch, wäre diese Forschung unmöglich gewesen.

Mijn collegae, Drs.C.P. Degenaar, Ing L.H.M. Habets, Dr.B.K. van Kreel; Cees "Lucien en Bernhard wil ik bedanken voor de gelegenheid, die zij mij de afgelopen maenden geboden hebben om dit proefschrift te kunnen afronden.

Een speciaal woord van dank aan Jasper Holthuis (en indirect aan Susanne), die de gratische verzorging op zich genomen heeft.

Cécile, Vincent, Paul en Yves, ons verlangen om heel bewust met en voor elkaar te leven kon in de afgelopen tijd maar nauwelijks gestalte krijgen. Nu deze druk weg is, zal ik ervoor zorgen, clat er weer alle gelegenheid is om samen allerlei dingen te ondernernen. Marie-Francoise, la dernière comme toujours! Et pourtant, si l'on se rends compte de l'importance de la stimulation et l"encouragement que j'ai reçu de toi, tu meriterais mieux. En plus, sans ta patience ce livre n"aurait jamais été terminé. Je ne la mettrai plus à l'épreuve; au contraire, j'essayerai de profiter plus du bonheur de nôtre union. Un très grand Merci. 


\section{Curriculem Vitae}

Paul Menheere is geboren op 11 maart 1950 te Venray.

Van 1962 tot 1967 werd het voorbereidend wetenschappelijk onderwijs genoten aan het Titus Brandsma Lyceum (HBS-B) te Oss.

Vanaf 1967 volgde hij de studie in de scheikundige technologie aan de Technische Universiteit Eindhoven. Vanaf januari 1973 tot juli 1974 werd het afstudeenwerk verricht in het 'Laboratoire de Chimie Analytique Physique' van de Ecole Polytechnique te Parijs.

In juni 1974 werd de studie algesloten met het behalen van het doctoraal examen aan de Technische Universiteit Eindhoven, afdeling der Scheikundige Technologie.

In 1975 werd een dienstverband aangegaan met het Sint Annaziekenhuis te Geldrop. In 1976 werd aldaar een aanvang gemaakt met de opleiding tot klinisch chemicus, die werd afgesloten met de inschrijving in het Register van Erkend Klinisch Chemici in januari 1982.

Vanaf februari 1982 tot juli 1985 had de auteur een dienstverband met de Nederlandse Hervormde Diaconessen Inrichting in Meppel. Hij was part-time gedetacheerd bij de Stichting SAZINON, eveneens te Meppel.

Sinds 1 juli 1985 is hij werkzaam als erkend klinisch chemicus in het Academisch Ziekenhuis Maastricht, speciaal belast met dle leiding van het immunochemisch laboratorium ten behoeve van het endocrinologisch en allergologisch onderzoek. 